\title{
Furan-2,5- and furan-2,3-dicarboxylate esters derived from marine biomass, as plasticizers for poly(vinyl chloride)
}

TanPhat Nguyen, ${ }^{\dagger, \ddagger}$ Yong Jin Kim, ${ }^{\dagger, \ddagger}$ Seok-Kyu Park, ${ }^{\dagger, \S}$ Kwan-Young Lee, ${ }^{\S}$ Ji-won Park," Jin Ku Cho, ${ }^{\dagger, \ddagger}$ and Seunghan Shin ${ }^{\dagger, \neq *}$

${ }^{\dagger}$ Green Chemistry \& Material Research Group, Korea Institute of Industrial Technology (KITECH), 89 Yangdaegiro-gil, Ipjang-myeon, Seobuk-ku, Cheonan, Chungnam 31056, Korea.

${ }^{\ddagger}$ Department of Green Process and System Engineering, Korea University of Science \& Technology (UST), 217 Gajeong-ro, Yuseong-gu, Daejeon 34113, Korea

${ }^{\S}$ Department of Chemical and Biological Engineering, Korea University, 145 Anam-ro, Seongbuk-gu, Seoul 02841, Korea

\footnotetext{
" Program in Environmental Materials Science, Seoul National University (SNU), 1 Gwanak-ro, Gwanak-gu, Seoul 08826, Korea
} 


\section{CONTENTS}

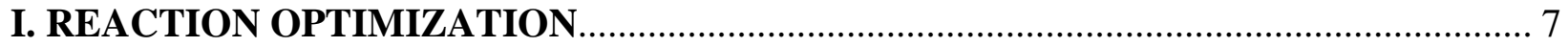

II. SPECTRA FOR STRUCTURAL CHARACTERIZATION ........................................ 8

1. Dialkyl furan-2,5-dicarboxylates (2,5-DAFs) .................................................................. 26

2. Dialkyl furan-2,3-dicarboxylates (2,3-DAFs) ................................................................. 40

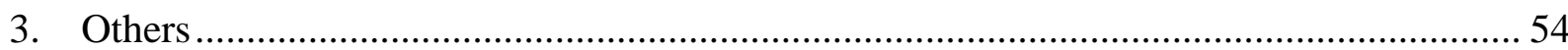

III. PREPARATION OF PLASTICIZED FILMS ………................................................ 58

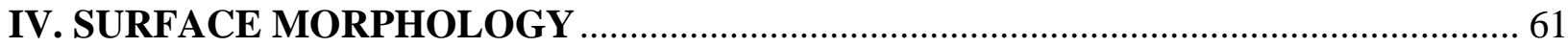

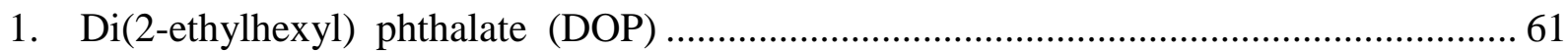

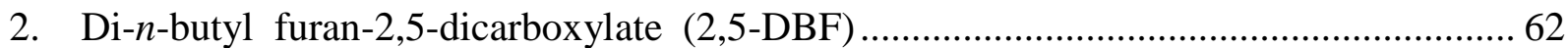

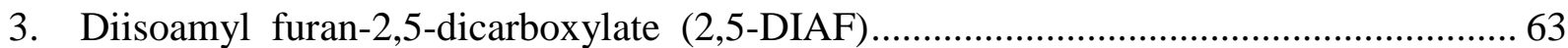

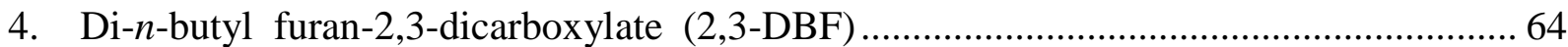

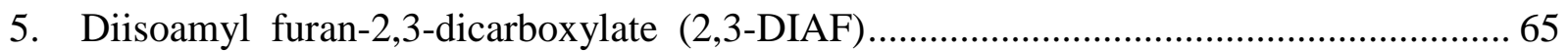

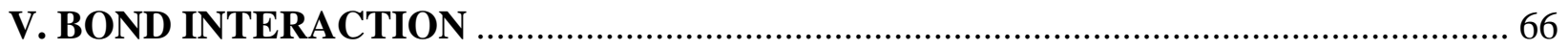

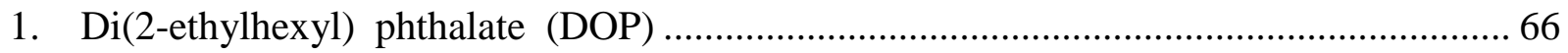

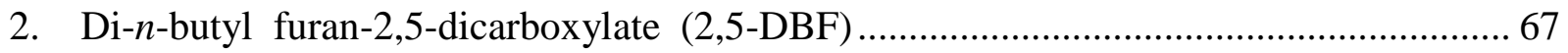

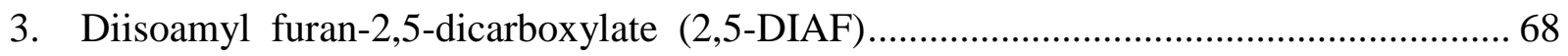

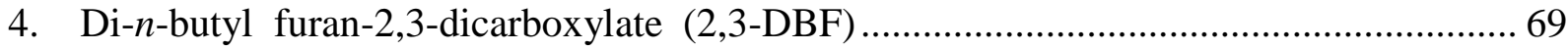

5. Diisoamyl furan-2,3-dicarboxylate (2,3-DIAF) ............................................................. 70

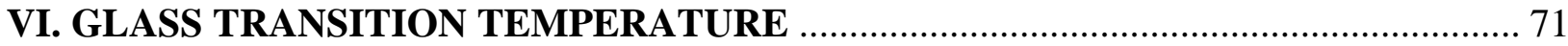

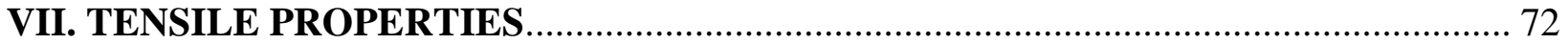

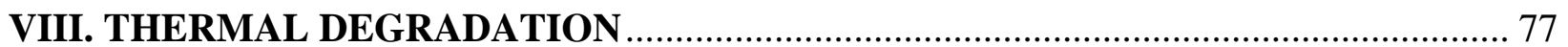

1. Di-n-butyl furan-2,5-dicarboxylate (2,5-DBF) ............................................................ 77

2. Diisoamyl furan-2,5-dicarboxylate (2,5-DIAF) ........................................................... 78

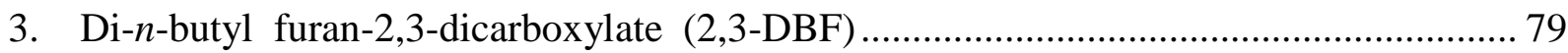

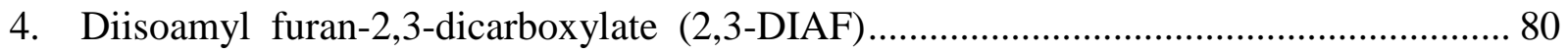

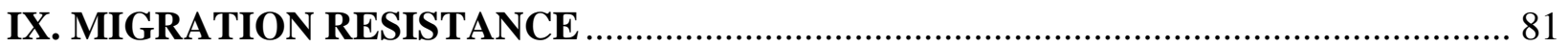




\section{LIST OF TABLES}

Table S1. Optimization for dehydration of galactaric acid, in alcoholic media with $\mathrm{H}_{2} \mathrm{SO}_{4}$........ 7

Table S2. Chemical-shift comparison between 2,5-DAFs, only selected is shown (in ppm) ...... 16

Table S3. Chemical-shift comparison between 2,3-DAFs, only selected is shown (in ppm)...... 17

Table S4. Fragmentation-pattern comparison between 2,5-DAF compounds, in m/z............... 22

Table S5. Fragmentation-pattern comparison between 2,3-DAF compounds, in m/z............... 23

Table S6. Infrared absorption comparison between 2,5-DAF compounds, in wavenumber $\left(\mathrm{cm}^{-1}\right)$

Table S7. Infrared absorption comparison between 2,3-DAF compounds, in wavenumber $\left(\mathrm{cm}^{-1}\right)$

Table S8. Formulations for preparation of PVC-PLS films ................................................. 59

Table S9. Differences in wavenumber of PVC-DOP films compared to pure DOP .................. 66

Table S10. Differences in wavenumber of PVC-2,5-DBF films compared to pure 2,5-DBF ..... 67

Table S11. Differences in wavenumber of PVC-2,5-DIAF films compared to pure 2,5-DIAF .. 68

Table S12. Differences in wavenumber of PVC-2,3-DBF films compared to pure 2,3-DBF ..... 69

Table S13. Differences in wavenumber of PVC-2,3-DIAF films compared to pure 2,3-DIAF .. 70

Table S14. Glass transition temperatures of PVC-PLS films ............................................... 71

Table S15. Tensile properties of investigated PVC-PLS films ............................................... 75

Table S16. Thermal degradation data of PVC-2,5-DBF films.............................................. 77

Table S17. Thermal degradation data of PVC-2,5-DIAF films ........................................... 78

Table S18. Thermal degradation data of PVC-2,3-DBF films............................................... 79

Table S19. Thermal degradation data of PVC-2,3-DIAF films ............................................ 80

Table S20. Table of data from extractable and volatile tests of all investigated plasticizers ....... 82 


\section{LIST OF FIGURES}

Figure S1. Structure depiction and position-naming of 2,5- and 2,3-DAFs ................................ 15

Figure S2. Proposed fragmentation pathway of 2,5-DAFs in mass spectroscopy (EI-MS)......... 18

Figure S3. Proposed initial events happened in an aliphatic branch of 2,3-DAF in EI-MS ........ 20

Figure S4. Proposed fragmentation pathway of 2,3-DAFs in mass spectroscopy (EI-MS)......... 20

Figure S5. Fragmentation pathway of alkyl 2-furoate in mass spectroscopy (EI-MS)................. 21

Figure S6. ${ }^{1} \mathrm{H}-\mathrm{NMR}$ spectrum of dibutyl furan-2,5-dicarboxylate (2,5-DBF) .............................. 26

Figure S7. ${ }^{13} \mathrm{C}$-NMR spectrum of dibutyl furan-2,5-dicarboxylate (2,5-DBF) .............................. 26

Figure S8. Infrared spectrum of dibutyl furan-2,5-dicarboxylate (2,5-DBF) ............................. 27

Figure S9. Mass spectrum of dibutyl furan-2,5-dicarboxylate (2,5-DBF) ……………………..... 27

Figure S10. ${ }^{1} \mathrm{H}-\mathrm{NMR}$ spectrum of dipentyl furan-2,5-dicarboxylate........................................... 28

Figure S11. ${ }^{13}$ C-NMR spectrum of dipentyl furan-2,5-dicarboxylate ........................................... 28

Figure S12. Infrared spectrum of dipentyl furan-2,5-dicarboxylate .............................................. 29

Figure S13. Mass spectrum of dipentyl furan-2,5-dicarboxylate .................................................. 29

Figure S14. ${ }^{1} \mathrm{H}-\mathrm{NMR}$ spectrum of dihexyl furan-2,5-dicarboxylate............................................. 30

Figure S15. ${ }^{13}$ C-NMR spectrum of dihexyl furan-2,5-dicarboxylate................................................ 30

Figure S16. Infrared spectrum of dihexyl furan-2,5-dicarboxylate .............................................. 31

Figure S17. Mass spectrum of dihexyl furan-2,5-dicarboxylate.................................................... 31

Figure S18. ${ }^{1} \mathrm{H}-\mathrm{NMR}$ spectrum of diheptyl furan-2,5-dicarboxylate............................................ 32

Figure S19. ${ }^{13}$ C-NMR spectrum of diheptyl furan-2,5-dicarboxylate ............................................. 32

Figure S20. Infrared spectrum of diheptyl furan-2,5-dicarboxylate ............................................... 33

Figure S21. Mass spectrum of diheptyl furan-2,5-dicarboxylate .................................................. 33

Figure S22. ${ }^{1} \mathrm{H}-\mathrm{NMR}$ spectrum of dioctyl furan-2,5-dicarboxylate............................................... 34

Figure S23. ${ }^{13}$ C-NMR spectrum of dioctyl furan-2,5-dicarboxylate ................................................ 34

Figure S24. Infrared spectrum of dioctyl furan-2,5-dicarboxylate ................................................ 35

Figure S25. Mass spectrum of dioctyl furan-2,5-dicarboxylate .................................................. 35

Figure S26. ${ }^{1}$ H-NMR spectrum of diisoamyl furan-2,5-dicarboxylate (2,5-DIAF) ...................... 36

Figure S27. ${ }^{13} \mathrm{C}$-NMR spectrum of diisoamyl furan-2,5-dicarboxylate (2,5-DIAF) ..................... 36

Figure S28. Infrared spectrum of diisoamyl furan-2,5-dicarboxylate (2,5-DIAF) ........................ 37

Figure S29. Mass spectrum of diisoamyl furan-2,5-dicarboxylate (2,5-DIAF) ............................ 37

Figure S30. ${ }^{1} \mathrm{H}-\mathrm{NMR}$ spectrum of di(2-ethylhexyl) furan-2,5-dicarboxylate................................ 38 
Figure S31. ${ }^{13} \mathrm{C}-\mathrm{NMR}$ spectrum of di(2-ethylhexyl) furan-2,5-dicarboxylate............................. 38

Figure S32. Infrared spectrum of di(2-ethylhexyl) furan-2,5-dicarboxylate ............................. 39

Figure S33. Mass spectrum of di(2-ethylhexyl) furan-2,5-dicarboxylate................................. 39

Figure S34. ${ }^{1}$ H-NMR spectrum of dibutyl furan-2,3-dicarboxylate (2,3-DBF)......................... 40

Figure S35. ${ }^{13} \mathrm{C}-\mathrm{NMR}$ spectrum of dibutyl furan-2,3-dicarboxylate (2,3-DBF) ........................ 40

Figure S36. Infrared spectrum of dibutyl furan-2,3-dicarboxylate (2,3-DBF) ........................ 41

Figure S37. Mass spectrum of dibutyl furan-2,3-dicarboxylate (2,3-DBF) ............................ 41

Figure S38. ${ }^{1}$ H-NMR spectrum of dipentyl furan-2,3-dicarboxylate........................................ 42

Figure S39. ${ }^{13}$ C-NMR spectrum of dipentyl furan-2,3-dicarboxylate ................................... 42

Figure S40. Infrared spectrum of dipentyl furan-2,3-dicarboxylate ...................................... 43

Figure S41. Mass spectrum of dipentyl furan-2,3-dicarboxylate ............................................ 43

Figure S42. ${ }^{1}$ H-NMR spectrum of dihexyl furan-2,3-dicarboxylate......................................... 44

Figure S43. ${ }^{13}$ C-NMR spectrum of dihexyl furan-2,3-dicarboxylate...................................... 44

Figure S44. Infrared spectrum of dihexyl furan-2,3-dicarboxylate ........................................ 45

Figure S45. Mass spectrum of dihexyl furan-2,3-dicarboxylate............................................. 45

Figure S46. ${ }^{1}$ H-NMR spectrum of diheptyl furan-2,3-dicarboxylate....................................... 46

Figure S47. ${ }^{13} \mathrm{C}-\mathrm{NMR}$ spectrum of diheptyl furan-2,3-dicarboxylate ..................................... 46

Figure S48. Infrared spectrum of diheptyl furan-2,3-dicarboxylate ...................................... 47

Figure S49. Mass spectrum of diheptyl furan-2,3-dicarboxylate ......................................... 47

Figure S50. ${ }^{1} \mathrm{H}-\mathrm{NMR}$ spectrum of dioctyl furan-2,3-dicarboxylate......................................... 48

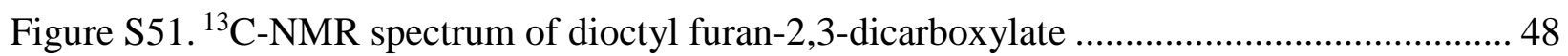

Figure S52. Infrared spectrum of dioctyl furan-2,3-dicarboxylate ....................................... 49

Figure S53. Mass spectrum of dioctyl furan-2,3-dicarboxylate ............................................. 49

Figure S54. ${ }^{1}$ H-NMR spectrum of diisoamyl furan-2,3-dicarboxylate (2,3-DIAF)................... 50

Figure S55. ${ }^{13} \mathrm{C}-\mathrm{NMR}$ spectrum of diisoamyl furan-2,3-dicarboxylate (2,3-DIAF) ................... 50

Figure S56. Infrared spectrum of diisoamyl furan-2,3-dicarboxylate (2,3-DIAF) .................... 51

Figure S57. Mass spectrum of diisoamyl furan-2,3-dicarboxylate (2,3-DIAF) ......................... 51

Figure S58. ${ }^{1} \mathrm{H}-\mathrm{NMR}$ spectrum of di(2-ethylhexyl) furan-2,3-dicarboxylate............................. 52

Figure S59. ${ }^{13}$ C-NMR spectrum of di(2-ethylhexyl) furan-2,3-dicarboxylate............................ 52

Figure S60. Infrared spectrum of di(2-ethylhexyl) furan-2,3-dicarboxylate ............................ 53

Figure S61. Mass spectrum of di(2-ethylhexyl) furan-2,3-dicarboxylate................................. 53 


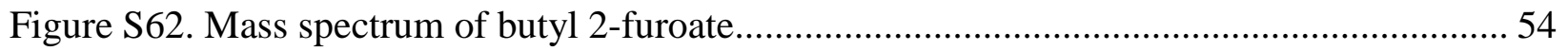

Figure S63. Mass spectrum of pentyl 2-furoate....................................................................... 54

Figure S64. Mass spectrum of hexyl 2-furoate......................................................................... 55

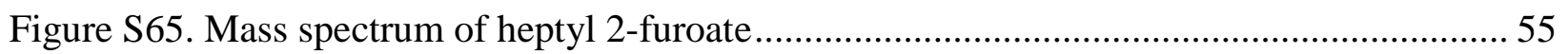

Figure S66. Mass spectrum of octyl 2-furoate............................................................................. 56

Figure S67. Mass spectrum of isoamyl 2-furoate …………………………………….................. 56

Figure S68. Mass spectrum of 2-ethylhexyl 2-furoate...................................................................... 57

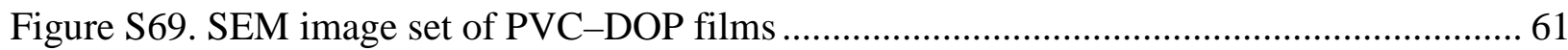

Figure S70. SEM image set of PVC-2,5-DBF films ................................................................. 62

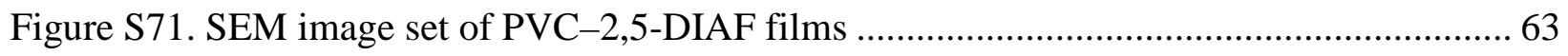

Figure S72. SEM image set of PVC-2,3-DBF films .................................................................. 64

Figure S73. SEM image set of PVC-2,3-DIAF films ................................................................ 65

Figure S74. IR absorption bands of pure DOP, and PVC-DOP films ........................................... 66

Figure S75. IR absorption bands of pure 2,5-DBF, and PVC-2,5-DBF films ............................ 67

Figure S76. IR absorption bands of pure 2,5-DIAF, and PVC-2,5-DIAF films ........................... 68

Figure S77. IR absorption bands of pure 2,3-DBF, and PVC-2,3-DBF films .............................. 69

Figure S78. IR absorption bands of pure 2,3-DIAF, and PVC-2,3-DIAF films ........................... 70

Figure S79. DSC thermograms of PVC and PVC-PLS films..................................................... 72

Figure S80. Thermal degradation curves of PVC-2,5-DBF films................................................. 77

Figure S81. Thermal degradation curves of PVC-2,5-DIAF films ............................................... 78

Figure S82. Thermal degradation curves of PVC-2,3-DBF films................................................. 79

Figure S83. Thermal degradation curves of PVC-2,3-DIAF films ................................................. 80 


\section{REACTION OPTIMIZATION}

Table S1. Optimization for dehydration of galactaric acid, in alcoholic media with $\mathrm{H}_{2} \mathrm{SO}_{4}$.

\begin{tabular}{|c|c|c|c|c|c|c|c|}
\hline \multirow[t]{2}{*}{ Entry } & \multicolumn{4}{|c|}{ Starting materials (g) } & \multicolumn{3}{|c|}{ Products } \\
\hline & Gal-dA & Alcohol & $\mathrm{H}_{2} \mathrm{SO}_{4}$ & p-xylene & $\begin{array}{c}\% \\
2,3-\mathrm{DAF}\end{array}$ & $\begin{array}{c}\% \\
2,5-\mathrm{DAF}\end{array}$ & $\begin{array}{c}\% \\
\text { in total }\end{array}$ \\
\hline 1 & & n-butyl alcohol, 24.00 & & & 30.08 & 63.07 & 93.15 \\
\hline 2 & & n-pentyl alcohol, 28.54 & & & 22.68 & 62.47 & 85.15 \\
\hline 3 & & n-hexyl alcohol, 33.08 & & & 21.03 & 53.73 & 74.76 \\
\hline 4 & 3.00 & n-heptyl alcohol, 37.63 & 1.2000 & 3.50 & 20.83 & 39.75 & 60.58 \\
\hline 5 & & n-octyl alcohol, 42.17 & & & 16.46 & 35.28 & 51.74 \\
\hline 6 & & isoamyl alcohol, 28.54 & & & 31.43 & 62.23 & 93.66 \\
\hline 7 & & 2-ethylhexyl alcohol, 42.17 & & & 10.87 & 34.98 & 45.85 \\
\hline 8 & & & 1.0000 & & 3.61 & 34.80 & 38.41 \\
\hline 9 & & & 1.5000 & & 20.40 & 30.59 & 50.99 \\
\hline 10 & & & 2.0000 & & 32.05 & 47.59 & 79.64 \\
\hline 11 & & & 2.5000 & & 30.64 & 63.20 & 93.84 \\
\hline 12 & 5.00 & n-butyl alcohol, 40.00 & 3.0000 & 5.00 & 39.61 & 55.11 & 94.72 \\
\hline 13 & & & 3.5000 & & 40.21 & 53.82 & 94.03 \\
\hline 14 & & & 4.0000 & & 30.96 & 61.21 & 92.17 \\
\hline 15 & & & 4.5000 & & 37.81 & 51.25 & 89.06 \\
\hline 16 & & & 5.0000 & & 38.53 & 51.54 & 90.07 \\
\hline 9 & & & & 5.00 & 20.40 & 30.59 & 50.99 \\
\hline 17 & & & & 5.50 & 26.52 & 52.38 & 78.90 \\
\hline 18 & 5.00 & n-butyl alcohol, 40.00 & 1.5000 & 6.00 & 25.06 & 70.09 & 95.15 \\
\hline 19 & & & & 6.50 & 24.81 & 46.05 & 70.86 \\
\hline 20 & & & & 7.00 & 15.38 & 38.61 & 53.99 \\
\hline
\end{tabular}




\section{SPECTRA FOR STRUCTURAL CHARACTERIZATION}

Nuclear magnetic resonance (NMR) spectra were recorded using a Bruker Spectrospin 300 (Germany), using chloroform- $d\left(\mathrm{CDCl}_{3}\right)$, and deuterated tetramethylsilane (TMS, $\delta=0 \mathrm{ppm}$ ) as internal standards. $\left({ }^{1} \mathrm{H}-\&{ }^{13} \mathrm{C}-\mathrm{NMR}\right.$; $300 \mathrm{MHz}$ and $75 \mathrm{MHz}$, respectively).

Infrared spectra (FTIR) were recorded using Nicolet 6700 FT-IR Spectrometer (Thermo Fisher, USA) with 32 scans, $4 \mathrm{~cm}^{-1}$ resolution and data spacing being $1.928 \mathrm{~cm}^{-1}$. It is known Fermi resonance is an important phenomenon to vibrational spectroscopies (IR or Raman). ${ }^{1}$ Also, conjugation between aromatic furan ring and carbonyl groups is unique and it leads to major frequency shifts, and intensity changes for both carbonyl and ring absorption bands.

Electron Ionization Mass spectroscopy (EI-MS +) spectra, were recorded on a Shimadzu GCMS-QP-2010 Ultra integrated with a Shimadzu GC for Mass Spectrometer GC-2010 Plus (Japan). In mass spectrometry, McLafferty rearrangement is an important phenomenon that helps interpreting the fragmentation, especially in the case of ester compound. ${ }^{1}$

Melting points were determined by temperature-scanning on a differential scanning calorimetry system (DSC 8000, PerkinElmer, USA). Crystal-form substances with weight in 2-5 mg and/or liquid ones with weight in $10-15 \mathrm{mg}$, were scanned slowly at a rate of $0.5^{\circ} \mathrm{C} / \mathrm{min}$.

Selected data of investigated compounds were summarized into tables, with some short explanations. Table S2 and Table S3 were created for NMR data of 2,5- and 2,3-DAFs, 
respectively. About EI-MS data, it was about Table S4 and Table S5. About FTIR data, it was about Table S6 and Table S7.

Di-n-butyl furan-2,5-dicarboxylate (2,5-DBF or F425). Mp 41.21-42.07 ${ }^{\circ} \mathrm{C}$; FTIR (neat) $v_{\max }$ $\left(\mathrm{cm}^{-1}\right)$ Csp $2-H:$ 3155, 3118, 771, Csp $_{3}-\mathrm{H}: 2958,2933,2871,1475,1383,735, \mathrm{C}=\mathrm{O}: 1724,1709$, ring deform.: 1576, 1510, C-O: 1282, 1230, 1151; ${ }^{1} \mathrm{H}$ NMR (300 MHz, $\left.\mathrm{CDCl}_{3}, \mathrm{Me}_{4} \mathrm{Si}\right) \delta 7.18$ (1H, s), 4.32 (2H, t, $\left.{ }^{3} J=6.7 \mathrm{~Hz}\right), 1.73$ (2H, quintet, $\left.{ }^{3} J=7.1 \mathrm{~Hz}\right), 1.43$ (2H, sextet, $\left.{ }^{3} J=7.3 \mathrm{~Hz}\right), 0.95$ (3H, t, $\left.{ }^{3} J=7.4 \mathrm{~Hz}\right) ;{ }^{13} \mathrm{C}$ NMR $\left(75 \mathrm{MHz}, \mathrm{CDCl}_{3}, \mathrm{Me}_{4} \mathrm{Si}\right) \delta 158.31,147.07,118.30,65.52,30.74$, 19.21, 13.80; MS (EI) m/z 268 (M+1.4 \%), 269 (0.2), 213 (8.0), 195 (26.6), 167 (9.9), 157 (100), 156 (19.3), 139 (83.8), 128 (1.5), 112 (7.1), 95 (13.0), 56 (58.0).

Di-n-pentyl furan-2,5-dicarboxylate (F525). Mp 9.42-11.06 ${ }^{\circ} \mathrm{C}$; FTIR (neat) $v_{\max }\left(\mathrm{cm}^{-1}\right) \mathrm{Csp}_{2}-$ H: 3161, 3128, 766, Csp3-H: 2958, 2933, 2871, 1468, 1379, 731, C=O: 1739, 1718, ring deform.: 1581, 1508, C-O: 1271, 1223, 1128; ${ }^{1} \mathrm{H}$ NMR (300 MHz, $\left.\mathrm{CDCl}_{3}, \mathrm{Me}_{4} \mathrm{Si}\right) \delta 7.19(1 \mathrm{H}, \mathrm{s}), 4.32(2 \mathrm{H}$, t, $\left.{ }^{3} J=6.8 \mathrm{~Hz}\right), 1.75\left(2 \mathrm{H}\right.$, quintet, $\left.{ }^{3} \mathrm{~J}=7.0 \mathrm{~Hz}\right), 1.46-1.29(4 \mathrm{H}, \mathrm{m}), 0.91\left(3 \mathrm{H}, \mathrm{t},{ }^{3} \mathrm{~J}=7.0 \mathrm{~Hz}\right) ;{ }^{13} \mathrm{C}$ NMR (75 MHz, $\mathrm{CDCl}_{3}, \mathrm{Me}_{4} \mathrm{Si}$ ) $\delta 158.22,146.99,118.26,65.72,28.34,28.03,22.35,13.98 ; \mathrm{MS}$ (EI) m/z 296 (M+, 0.5 \%), 297 (0.1), 227 (19.8), 209 (25.5), 181 (8.5), 157 (100), 156 (18.1), 139 (92.2), 128 (1.3), 112 (7.0), 95 (14.8), 70 (52.8).

Di-n-hexyl furan-2,5-dicarboxylate (F625). Mp 29.60-30.64 ${ }^{\circ} \mathrm{C}$; FTIR (neat) $v_{\max }\left(\mathrm{cm}^{-1}\right)$ Csp2-H: 3151, 3116, 773, Сsp $-H$ : 2954, 2933, 2870, 2854, 1469, 1377, 725, C=O: 1724, ring deform.: 1574, 1508, C-O: 1275, 1230, 1157; ${ }^{1} \mathrm{H}$ NMR (300 MHz, CDCl, $\left.\mathrm{Me}_{4} \mathrm{Si}\right) \delta 7.14$ (1H, s), $4.26\left(2 \mathrm{H}, \mathrm{t},{ }^{3} \mathrm{~J}=6.8 \mathrm{~Hz}\right), 1.68\left(2 \mathrm{H}\right.$, quintet, $\left.{ }^{3} \mathrm{~J}=7.0 \mathrm{~Hz}\right), 1.42-1.16(6 \mathrm{H}, \mathrm{m}), 0.82\left(3 \mathrm{H}, \mathrm{t},{ }^{3} \mathrm{~J}=6.6 \mathrm{~Hz}\right)$; 
${ }^{13} \mathrm{C}$ NMR (75 MHz, $\left.\mathrm{CDCl}_{3}, \mathrm{Me}_{4} \mathrm{Si}\right) \delta 158.09,146.91,118.17,65.60,31.36,28.52,25.48,22.48$, 13.93; MS (EI) m/z 324 (M+, 0.5 \%), 325 (0.1), 241 (29.6), 223 (14.3), 195 (7.4), 157 (100), 156 (15.1), 139 (92.4), 128 (1.1), 112 (6.3), 95 (15.6), 84 (23.5), 56 (65.7).

Di-n-heptyl furan-2,5-dicarboxylate (F725). Mp 14.19-16.03 ${ }^{\circ} \mathrm{C}$; FTIR (neat) $v_{\max }\left(\mathrm{cm}^{-1}\right)$ Csp $2-H:$ 3163, 3128, 766, Csp $3-H:$ 2954, 2927, 2857, 1468, 1377, 725, C=O: 1739, 1720, ring deform.: 1581, 1508, C-O: 1273, 1221, 1128; ${ }^{1} \mathrm{H}$ NMR (300 MHz, CDCl 3 , Me 4 Si) $\delta 7.16$ (1H, s), $4.29\left(2 \mathrm{H}, \mathrm{t},{ }^{3} \mathrm{~J}=6.8 \mathrm{~Hz}\right), 1.72\left(2 \mathrm{H}\right.$, quintet, $\left.{ }^{3} \mathrm{~J}=7.1 \mathrm{~Hz}\right), 1.44-1.19(8 \mathrm{H}, \mathrm{m}), 0.85\left(3 \mathrm{H}, \mathrm{t},{ }^{3} \mathrm{~J}=6.9 \mathrm{~Hz}\right)$; ${ }^{13} \mathrm{C}$ NMR $\left(75 \mathrm{MHz}, \mathrm{CDCl}_{3}, \mathrm{Me}_{4} \mathrm{Si}\right) \delta 158.20,147.02,118.23,65.72,31.74,28.94,28.66,25.86$, 22.62, 14.08; MS (EI) m/z 352 (M+, 0.4 \%), 353 (0.1), 255 (31.0), 237 (3.3), 209 (6.5), 157 (100), 156 (12.5), 139 (76.4), 128 (0.8), 113 (6.1), 112 (5.4), 98 (15.2), 95 (13.5), 70 (49.3).

Di-n-octyl furan-2,5-dicarboxylate (F825). Mp 47.49-48.38 ${ }^{\circ} \mathrm{C}$; FTIR (neat) $v_{\max }\left(\mathrm{cm}^{-1}\right)$ Csp2-H: 3151, 3118, 775, Csp3-H: 2952, 2920, 2854, 1469, 723, C=O: 1726, ring deform.: 1574, 1506, C-O: 1273, 1232, 1153; ${ }^{1} \mathrm{H}$ NMR (300 MHz, CDCl, $\left.\mathrm{Me}_{4} \mathrm{Si}\right) \delta 7.14(1 \mathrm{H}, \mathrm{s}), 4.27$ (2H, t, $\left.{ }^{3} J=6.8 \mathrm{~Hz}\right), 1.70\left(2 \mathrm{H}\right.$, quintet, $\left.{ }^{3} J=7.1 \mathrm{~Hz}\right), 1.43-1.14(10 \mathrm{H}, \mathrm{m}), 0.83\left(3 \mathrm{H}, \mathrm{t},{ }^{3} J=6.8 \mathrm{~Hz}\right) ;{ }^{13} \mathrm{C}$ NMR (75 MHz, $\mathrm{CDCl}_{3}, \mathrm{Me}_{4} \mathrm{Si}$ ) $\delta 158.13,146.97,118.18,65.65,31.78,29.20,29.17,28.61,25.86,22.64$, 14.06; MS (EI) m/z 380 (M+1, 0.5 \%), 381 (0.1), 269 (45.7), 251 (1.8), 223 (11.1), 157 (100), 156 (13.7), 139 (74.5), 128 (0.9), 113 (25.1), 112 (17.1), 95 (13.8), 84 (27.9).

Diisoamyl furan-2,5-dicarboxylate, aka di-i-pentyl furan-2,5-dicarboxylate (2,5-DIAF or F525i). Mp 35.48-36.24 ${ }^{\circ} \mathrm{C}$; FTIR (neat) $v_{\max }\left(\mathrm{cm}^{-1}\right) \mathrm{Csp}_{2}-\mathrm{H}: 3153,3118,771$, Csp $3-\mathrm{H}: 2956$, 2933, 2870, 1464, 1392, 1365, C=O: 1724, 1709, ring deform.: 1576, 1508, C-O: 1284, 1275, 
1232, 1153; ${ }^{1} \mathrm{H}$ NMR (300 MHz, $\left.\mathrm{CDCl}_{3}, \mathrm{Me}_{4} \mathrm{Si}\right) \delta 7.18(1 \mathrm{H}, \mathrm{s}), 4.35$ (2H, t, $\left.{ }^{3} \mathrm{~J}=6.8 \mathrm{~Hz}\right), 1.83-1.58$ (3H, m), 0.95 (6H, d, $\left.{ }^{3} J=6.5 \mathrm{~Hz}\right) ;{ }^{13} \mathrm{C}$ NMR $\left(75 \mathrm{MHz}, \mathrm{CDCl}_{3}, \mathrm{Me}_{4} \mathrm{Si}\right) \delta 158.31,147.07,118.32$, 64.37, 37.37, 25.21, 22.58; MS (EI) m/z 296 (M+1, 0.02 \%), 297 (0.01), 281 (0.3), 253 (0.6), 227 (6.2), 209 (21.9), 181 (2.9), 157 (28.7), 156 (5.0), 139 (62.2), 128 (0.5), 112 (2.5), 95 (9.2), 71 (74.4), 70 (100).

Di(2-ethylhexyl) furan-2,5-dicarboxylate (F825i). Mp 9.53-11.86 ${ }^{\circ} \mathrm{C}$; two recrystallization points in the range of $-75-0{ }^{\circ} \mathrm{C}$ : $\mathrm{Rp}_{1}-58.84$ to $-57.61{ }^{\circ} \mathrm{C}$, $\mathrm{Rp} 2-34.99$ to $-32.89^{\circ} \mathrm{C}$; FTIR (neat) $v_{\max }$ $\left(\mathrm{cm}^{-1}\right)$ Csp2-H: 3163, 3128, 766, Сsp3-H: 2958, 2929, 2873, 2860, 1463, 1381, 729, C=O: 1741, 1720, ring deform.: 1581, 1507, C-O: 1273, 1221, 1128; ${ }^{1} \mathrm{H}$ NMR (300 MHz, CDCl 3 , Me $\left.4 \mathrm{Si}\right) \delta$ 7.15 (1H, s), 4.20 (2H, ddd, $\left.{ }^{2} J=13.1 \mathrm{~Hz},{ }^{3} J=5.9 \mathrm{~Hz},{ }^{4} \mathrm{~J}=10.9 \mathrm{~Hz}\right), 1.67$ (1H, septet, ${ }^{3} \mathrm{~J}=6.0 \mathrm{~Hz}$ ), 1.47-1.19 (8H, m), $0.89 \& 0.85$ (6H, overlapped triplets, $\left.{ }^{3} \mathrm{~J}=7.5,7.0 \mathrm{~Hz}\right) ;{ }^{13} \mathrm{C}$ NMR $(75 \mathrm{MHz}$, $\left.\mathrm{CDCl}_{3}, \mathrm{Me} 4 \mathrm{Si}\right) \delta 158.25,147.02,118.13,67.89,38.85,30.42,28.95,23.85,22.97,14.04,11.01 ;$ MS (EI) m/z 380 (M+, 0.05 \%), 269 (5.7), 251 (5.7), 223 (1.5 ), 157 (65.2), 156 (0.8), 139 (71.9), 128 (0.1), 112 (45.0), 95 (9.8), 70 (100).

Di-n-butyl furan-2,3-dicarboxylate (2,3-DBF or F423). Mp 53.30-54.19 ${ }^{\circ} \mathrm{C}$; FTIR (neat) $v_{\max }$ $\left(\mathrm{cm}^{-1}\right)$ Csp2 $-\mathrm{H}: 3116,3076,758, \mathrm{Csp}_{3}-\mathrm{H}:$ 2960, 2875, 1475, 1383, 731, C=O: 1716, ring deform.: 1637, 1556, C-O: 1257, 1144, 1095; ${ }^{1} \mathrm{H}$ NMR (300 MHz, CDCl, $\left.\mathrm{Me}_{4} \mathrm{Si}\right) \delta 6.97\left(1 \mathrm{H}, \mathrm{d},{ }^{3} \mathrm{~J}=7.5\right.$ Hz), $6.46\left(1 \mathrm{H}, \mathrm{d},{ }^{3} \mathrm{~J}=7.6 \mathrm{~Hz}\right), 4.07\left(2 \mathrm{H}, \mathrm{t},{ }^{3} \mathrm{~J}=6.7 \mathrm{~Hz}\right), 3.83\left(2 \mathrm{H}, \mathrm{t},{ }^{3} \mathrm{~J}=6.5 \mathrm{~Hz}\right), 1.62$ (2H, quintet, $\left.{ }^{3} J=7.0 \mathrm{~Hz}\right), 1.50\left(2 \mathrm{H}\right.$, quintet, $\left.{ }^{3} \mathrm{~J}=7.1 \mathrm{~Hz}\right), 1.39-1.09(4 \mathrm{H}, \mathrm{m}), 0.743 \& 0.735$ (6H, overlapped triplets, $\left.{ }^{3} \mathrm{~J}=7.4,7.4 \mathrm{~Hz}\right) ;{ }^{13} \mathrm{C}$ NMR $\left(75 \mathrm{MHz}, \mathrm{CDCl}_{3}, \mathrm{Me}_{4} \mathrm{Si}\right) \delta 158.96,156.36,148.44,140.33$, 111.67, 111.08, 69.22, 65.26, 30.15, 30.09, 18.68, 18.67, 13.26, 13.22; MS (EI) m/z 268 (M+1, 5.4 
\%), 269 (0.8), 213 (6.9), 212 (12.3), 195 (2.4), 167 (50.1), 157 (34.6), 156 (100), 139 (12.4), 128 (51.4), 111 (11.3), 110 (14.8), 100 (15.8), 95 (7.0), 82 (71.8), 57 (77.1), 56 (29.0), 41 (97).

Di-n-pentyl furan-2,3-dicarboxylate (F523). Mp 48.80-50.90 ${ }^{\circ} \mathrm{C}$; FTIR (neat) $v_{\max }\left(\mathrm{cm}^{-1}\right)$ Csp2-H: 3117, 3076, 758, Сsp $-\mathrm{H}:$ 2954, 2933, 2871, 2862, 1477, 1381, 721, C=O: 1716, ring deform.: 1637, 1560, C-O: 1261, 1146, 1097; ${ }^{1} \mathrm{H}$ NMR (300 MHz, $\left.\mathrm{CDCl}_{3}, \mathrm{Me}_{4} \mathrm{Si}\right) \delta 7.08(1 \mathrm{H}$, d, $\left.{ }^{3} \mathrm{~J}=7.5 \mathrm{~Hz}\right), 6.47\left(1 \mathrm{H}, \mathrm{d},{ }^{3} \mathrm{~J}=7.6 \mathrm{~Hz}\right), 4.21\left(2 \mathrm{H}, \mathrm{t},{ }^{3} \mathrm{~J}=6.8 \mathrm{~Hz}\right), 3.93\left(2 \mathrm{H}, \mathrm{t},{ }^{3} \mathrm{~J}=6.6 \mathrm{~Hz}\right), 1.79(2 \mathrm{H}$, quintet, $\left.{ }^{3} \mathrm{~J}=7.0 \mathrm{~Hz}\right), 1.67$ (2H, quintet, $\left.{ }^{3} \mathrm{~J}=7.0 \mathrm{~Hz}\right), 1.44-1.22$ (8H, m), 0.89-0.80 (6H, multiplet); ${ }^{13} \mathrm{C}$ NMR $\left(75 \mathrm{MHz}, \mathrm{CDCl}_{3}, \mathrm{Me}_{4} \mathrm{Si}\right) \delta 159.44,156.72,148.87,140.87,111.73,111.04,69.85$, 66.07, 28.19, 28.14, 27.910, 27.906, 22.29, 22.24, 13.88, 13.87; MS (EI) m/z 296 (M+1 1.4 \%), 297 (0.4), 227 (8.2), 209 (0.7), 181 (22.2), 157 (34.0), 156 (49.8), 139 (7.2), 128 (16.9), 111 (5.5), 100 (5.0), 95 (2.9), 82 (25.5), 71 (17.1), 70 (17.3), 43 (100).

Di-n-hexyl furan-2,3-dicarboxylate (F623). Mp 53.82-55.12 ${ }^{\circ} \mathrm{C}$; FTIR (neat) $v_{\max }\left(\mathrm{cm}^{-1}\right)$ Csp2-H: 3116, 3076, 758, Сsp $-\mathrm{H}:$ 2956, 2918, 2873, 2858, 1475, 1383, 719, C=O: 1716, ring deform.: 1637, 1558, C-O: 1267, 1146, 1097; ${ }^{1} \mathrm{H}$ NMR (300 MHz, CDCl 3 , $\left.\mathrm{Me}_{4} \mathrm{Si}\right) \delta 7.08$ (1H, d, $\left.{ }^{3} J=7.5 \mathrm{~Hz}\right), 6.47\left(1 \mathrm{H}, \mathrm{d},{ }^{3} J=7.6 \mathrm{~Hz}\right), 4.21\left(2 \mathrm{H}, \mathrm{t},{ }^{3} \mathrm{~J}=6.8 \mathrm{~Hz}\right), 3.93\left(2 \mathrm{H}, \mathrm{t},{ }^{3} \mathrm{~J}=6.6 \mathrm{~Hz}\right), 1.79(2 \mathrm{H}$, quintet, $\left.{ }^{3} \mathrm{~J}=7.1 \mathrm{~Hz}\right), 1.66\left(2 \mathrm{H}\right.$, quintet, $\left.{ }^{3} \mathrm{~J}=7.0 \mathrm{~Hz}\right), 1.46-1.17(12 \mathrm{H}, \mathrm{m}), 0.82\left(6 \mathrm{H}, \mathrm{t},{ }^{3} \mathrm{~J}=7.0 \mathrm{~Hz}\right)$; ${ }^{13} \mathrm{C}$ NMR $\left(75 \mathrm{MHz}, \mathrm{CDCl}_{3}, \mathrm{Me}_{4} \mathrm{Si}\right) \delta 159.44,156.70,148.88,140.88,111.72,111.03,69.86$, 66.08, 31.39, 31.34, 28.45, 28.40, 25.48, 25.46, 22.47, 13.95; MS (EI) m/z 324 (M+1 1.2 \%), 325 (0.6), 241 (19.3), 223 (0.8), 195 (24.8), 157 (61.4), 156 (58.6), 139 (10.9), 128 (18.1), 111 (7.5), 100 (4.7), 95 (3.1), 85 (18.5), 84 (14.9), 82 (26.1), 43 (100). 
Di-n-heptyl furan-2,3-dicarboxylate (F723). Mp 61.54-63.14 ${ }^{\circ} \mathrm{C}$; FTIR (neat) $v_{\max }\left(\mathrm{cm}^{-1}\right)$ Csp2-H: 3116, 3077, 758, Сsp3-H: 2954, 2918, 2854, 1475, 1379, 717, C=O: 1716, ring deform.: 1637, 1560, C-O: 1265, 1146, 1097; ${ }^{1} \mathrm{H}$ NMR (300 MHz, CDCl, $\left.\mathrm{Me}_{4} \mathrm{Si}\right) \delta 7.08\left(1 \mathrm{H}, \mathrm{d},{ }^{3} \mathrm{~J}=7.5\right.$ Hz), $6.46\left(1 \mathrm{H}, \mathrm{d},{ }^{3} \mathrm{~J}=7.6 \mathrm{~Hz}\right), 4.22\left(2 \mathrm{H}, \mathrm{t},{ }^{3} \mathrm{~J}=6.8 \mathrm{~Hz}\right), 3.93\left(2 \mathrm{H}, \mathrm{t},{ }^{3} \mathrm{~J}=6.6 \mathrm{~Hz}\right), 1.80$ (2H, quintet, $\left.{ }^{3} \mathrm{~J}=7.1 \mathrm{~Hz}\right), 1.67\left(2 \mathrm{H}\right.$, quintet, $\left.{ }^{3} \mathrm{~J}=7.0 \mathrm{~Hz}\right), 1.46-1.14(16 \mathrm{H}, \mathrm{m}), 0.82\left(6 \mathrm{H}, \mathrm{t},{ }^{3} \mathrm{~J}=6.8 \mathrm{~Hz}\right) ;{ }^{13} \mathrm{C}$ NMR (75 MHz, $\left.\mathrm{CDCl}_{3}, \mathrm{Me}_{4} \mathrm{Si}\right) \delta 159.48,156.72,148.92,140.93,111.71,111.03,69.88,66.12,31.66$, 28.91, 28.86, 28.52, 28.47, 25.79, 22.55, 14.03; MS (EI) m/z 352 ( $\mathrm{M}^{+}, 0.5$ \%), 353 (0.2), 255 (17.3), 237 (0.4), 209 (11.8), 157 (45.6), 156 (33.5), 139 (7.0), 128 (8.5), 111 (4.5), 99 (2.5), 98 (6.9), 95 (2.0), 82 (14.7), 57 (100).

Di-n-octyl furan-2,3-dicarboxylate (F823). Mp 67.20-68.71 ${ }^{\circ} \mathrm{C}$; FTIR (neat) $v_{\max }\left(\mathrm{cm}^{-1}\right) \mathrm{Csp}_{2}-$ H: 3116, 3078, 760, Сspз-H: 2954, 2917, 2852, 1475, 1377, 717, C=O: 1716, ring deform.: 1637, 1560, C-O: 1269, 1146, 1097; ${ }^{1} \mathrm{H}$ NMR (300 MHz, CDCl 3 , Me $4 \mathrm{Si}$ ) $\delta 7.09$ (1H, d, $\left.{ }^{3} J=7.5 \mathrm{~Hz}\right), 6.46$ (1H, d, $\left.{ }^{3} \mathrm{~J}=7.6 \mathrm{~Hz}\right), 4.23\left(2 \mathrm{H}, \mathrm{t},{ }^{3} \mathrm{~J}=6.8 \mathrm{~Hz}\right), 3.94\left(2 \mathrm{H}, \mathrm{t},{ }^{3} \mathrm{~J}=6.6 \mathrm{~Hz}\right), 1.81$ (2H, quintet, $\left.{ }^{3} \mathrm{~J}=7.1 \mathrm{~Hz}\right)$, $1.68\left(2 \mathrm{H}\right.$, quintet, $\left.{ }^{3} \mathrm{~J}=7.0 \mathrm{~Hz}\right), 1.47-1.13(20 \mathrm{H}, \mathrm{m}), 0.83\left(6 \mathrm{H}, \mathrm{t},{ }^{3} \mathrm{~J}=6.7 \mathrm{~Hz}\right) ;{ }^{13} \mathrm{C}$ NMR $(75 \mathrm{MHz}$, $\left.\mathrm{CDCl}_{3}, \mathrm{Me} 4 \mathrm{Si}\right) \delta 159.52,156.75,148.95,140.96,111.71,111.01,69.91,66.16,31.78,29.24$, 29.19, 29.16, 28.55, 28.49, 25.86, 22.64, 14.09; MS (EI) m/z 380 (M+, 0.62 \%), 381 (0.56), 269 (39.7), 251 (0.6), 223 (18.1), 157 (78.1), 156 (48.5), 139 (11.7), 128 (13.0), 113 (3.4), 112 (10.6), 111 (8.3), 95 (2.9), 82 (24.8), 71 (63.6), 57 (100).

Diisoamyl furan-2,3-dicarboxylate, aka di-i-pentyl furan-2,3-dicarboxylate (2,3-DIAF or F523i). Mp 74.92-75.88 ${ }^{\circ} \mathrm{C}$; FTIR (neat) $v_{\max }\left(\mathrm{cm}^{-1}\right)$ Csp2-H: 3117, 3084, 762, Сsp $3-\mathrm{H}: 2953$, 2870, 1471, 1387, 1367, C=O: 1731, 1712, ring deform.: 1635, 1558, C-O: 1253, 1142, 1092; ${ }^{1} \mathrm{H}$ 
NMR (300 MHz, CDCl $\left.3, \mathrm{Me}_{4} \mathrm{Si}\right) \delta 7.11\left(1 \mathrm{H}, \mathrm{d},{ }^{3} \mathrm{~J}=7.5 \mathrm{~Hz}\right), 6.47$ (1H, d, $\left.{ }^{3} \mathrm{~J}=7.5 \mathrm{~Hz}\right), 4.32(2 \mathrm{H}, \mathrm{t}$, $\left.{ }^{3} \mathrm{~J}=6.8 \mathrm{~Hz}\right), 4.00\left(2 \mathrm{H}, \mathrm{t},{ }^{3} \mathrm{~J}=6.6 \mathrm{~Hz}\right), 1.93-1.57(6 \mathrm{H}, \mathrm{m}), 0.95$ \& 0.94 (12H, overlapped doublets, $\left.{ }^{3} J=6.4,6.5 \mathrm{~Hz}\right) ;{ }^{13} \mathrm{C}$ NMR $\left(75 \mathrm{MHz}, \mathrm{CDCl}_{3}, \mathrm{Me}_{4} \mathrm{Si}\right) \delta 159.48,156.71,148.91,140.93,111.72$, 111.05, 68.28, 64.63, 37.16, 37.02, 24.99, 24.86, 22.41; MS (EI) m/z $296\left(\mathrm{M}^{+}, 1.1\right.$ \%), 297 (0.3), 281 (0.2), 253 (0.2), 227 (9.6), 209 (0.9), 181 (1.6), 157 (15.2), 156 (9.3), 139 (5.7), 128 (1.9), 111 (3.3) 95 (1.8), 82 (10.4), 71 (62.4), 70 (57.5), 43 (100).

Di(2-ethylhexyl) furan-2,3-dicarboxylate (F823i). FTIR (neat) $v_{\max }\left(\mathrm{cm}^{-1}\right)$ Csp2-H: 3122, 3078, 760, Сsp3-H: 2958, 2929, 2873, 1463, 1381, 727, C=O: 1728, ring deform.: 1637, 1560, CO: 1246, 1128, 1080; ${ }^{1} \mathrm{H}$ NMR (300 MHz, $\left.\mathrm{CDCl}_{3}, \mathrm{Me}_{4} \mathrm{Si}\right) \delta 7.08\left(1 \mathrm{H}, \mathrm{d},{ }^{3} \mathrm{~J}=7.5 \mathrm{~Hz}\right), 6.46(1 \mathrm{H}, \mathrm{d}$, $\left.{ }^{3} \mathrm{~J}=7.6 \mathrm{~Hz}\right), 4.16\left(2 \mathrm{H}, \mathrm{ddd},{ }^{2} \mathrm{~J}=12.6 \mathrm{~Hz},{ }^{3} \mathrm{~J}=5.9 \mathrm{~Hz},{ }^{4} \mathrm{~J}=10.9 \mathrm{~Hz}\right), 3.82\left(2 \mathrm{H}, \mathrm{ddd},{ }^{2} \mathrm{~J}=11.3 \mathrm{~Hz},{ }^{3} \mathrm{~J}=6.0\right.$ $\left.\mathrm{Hz},{ }^{4} J=9.5 \mathrm{~Hz}\right), 1.79$ (1H, septet, $\left.{ }^{3} J=6.2 \mathrm{~Hz}\right), 1.65$ (1H, septet, $\left.{ }^{3} J=6.0 \mathrm{~Hz}\right), 1.56-1.14$ (16H, m), 0.94-0.77 (12H, m); ${ }^{13} \mathrm{C}$ NMR (75 MHz, $\left.\mathrm{CDCl}_{3}, \mathrm{Me}_{4} \mathrm{Si}\right) \delta 159.65,156.61,149.25,140.99,111.66$, 110.91, 72.43, 68.44, 38.81, 30.36, 30.24, 28.95, 23.78, 23.64, 22.99, 14.08, 10.99, 10.94; MS (EI) m/z 381 ( $\left.\mathrm{M}^{+}, 0.2 \%\right), 269$ (19.0), 251 (0.2), 223 (4.8), 157 (41.1), 156 (12.0), 139 (9.3), 128 (3.2), 113 (11.0), 112 (39.6), 111 (6.1), 95 (1.8), 83 (34.7), 71 (94.2), 57 (100).

NMR spectra provides the structural information of investigated compounds. Some specific positions are naming for easy categorization and comparison. Some aliphatic signals overlapping its twin signal and enhancing peak intensity, are marked with §. 


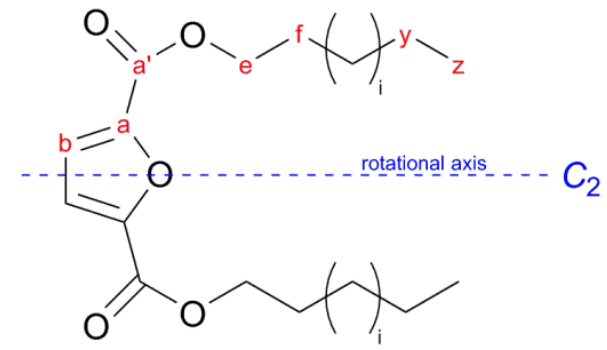

2,5-DAF

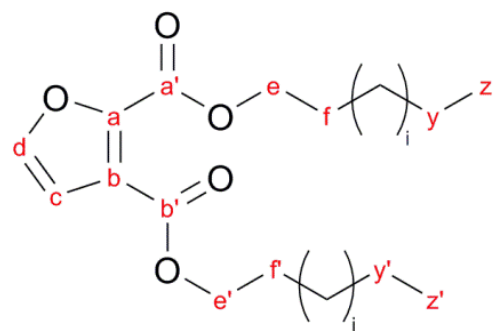

2,3-DAF

Figure S1. Structure depiction and position-naming of 2,5- and 2,3-DAFs

Because of symmetry, in the structure of 2,5-DAFs there are three kinds of nuclei which are related to the furandicarboxylic core, and $n$ kinds of nuclei from aliphatic moiety which is corresponding to the chain length. ${ }^{13} \mathrm{C}$ NMR spectra: furan core approximately $\delta 158$ ppm, 147 ppm, 118 ppm; and the aliphatic chain ranged 70-13 ppm. ${ }^{1} \mathrm{H}$ NMR spectra: approximately $\delta 7.2$ ppm (singlet, $\mathrm{H}_{\mathrm{b}}$ ), $4.3 \mathrm{ppm}$ (triplet, ${ }^{3} J=6.8 \mathrm{~Hz}, \mathrm{H}_{\mathrm{e}}$ ), $1.7 \mathrm{ppm}$ (quintet, ${ }^{3} J=7.0 \mathrm{~Hz}, \mathrm{H}_{\mathrm{f}}$ ); and 0.9 ppm (triplet, ${ }^{3} J=6.8 \mathrm{~Hz}, \mathrm{H}_{\mathrm{z}}$ ).

In 2,3-DAFs' structure there are six kinds of nuclei for the furandicarboxylic core, and $2 n$ kinds of nuclei from two aliphatic moieties. NMR data provides the fact that two aliphatic moieties are magnetically different and thereafter, signals usually appear in pair with one moiety being in the higher field compared to the other. ${ }^{13} \mathrm{C}$ NMR spectra: furan core approximately $\delta 159.5,156.5$, 149, 141, 112, 111; and the aliphatic chain appeared in pair and ranged 70-13 ppm. ${ }^{1} \mathrm{H}$ NMR spectra: furan core approximately $\delta 7.0$ ppm (doublet, ${ }^{3} J=7.5 \mathrm{~Hz}$ ), $6.5 \mathrm{ppm}$ (doublet, ${ }^{3} \mathrm{~J}=7.6 \mathrm{~Hz}$ ); and the aliphatic chain $4.2 \mathrm{ppm}$ (triplet, ${ }^{3} \mathrm{~J}=6.8 \mathrm{~Hz}, \mathrm{H}_{\mathrm{e}}$ ), $3.9 \mathrm{ppm}$ (triplet, ${ }^{3} J=6.6 \mathrm{~Hz}, \mathrm{H}_{\mathrm{e}}$ ), 1.8 ppm (quintet, ${ }^{3} J=7.0 \mathrm{~Hz}, \mathrm{H}_{\mathrm{f}}{ }^{\prime}$ ); $1.7 \mathrm{ppm}$ (quintet, ${ }^{3} J=7.0 \mathrm{~Hz}, \mathrm{H}_{\mathrm{f}}$ ); and $0.8^{\S} \mathrm{ppm}\left(\mathrm{H}_{\mathrm{z}}\right)$. 
Table S2. Chemical-shift comparison between 2,5-DAFs, only selected is shown (in ppm)

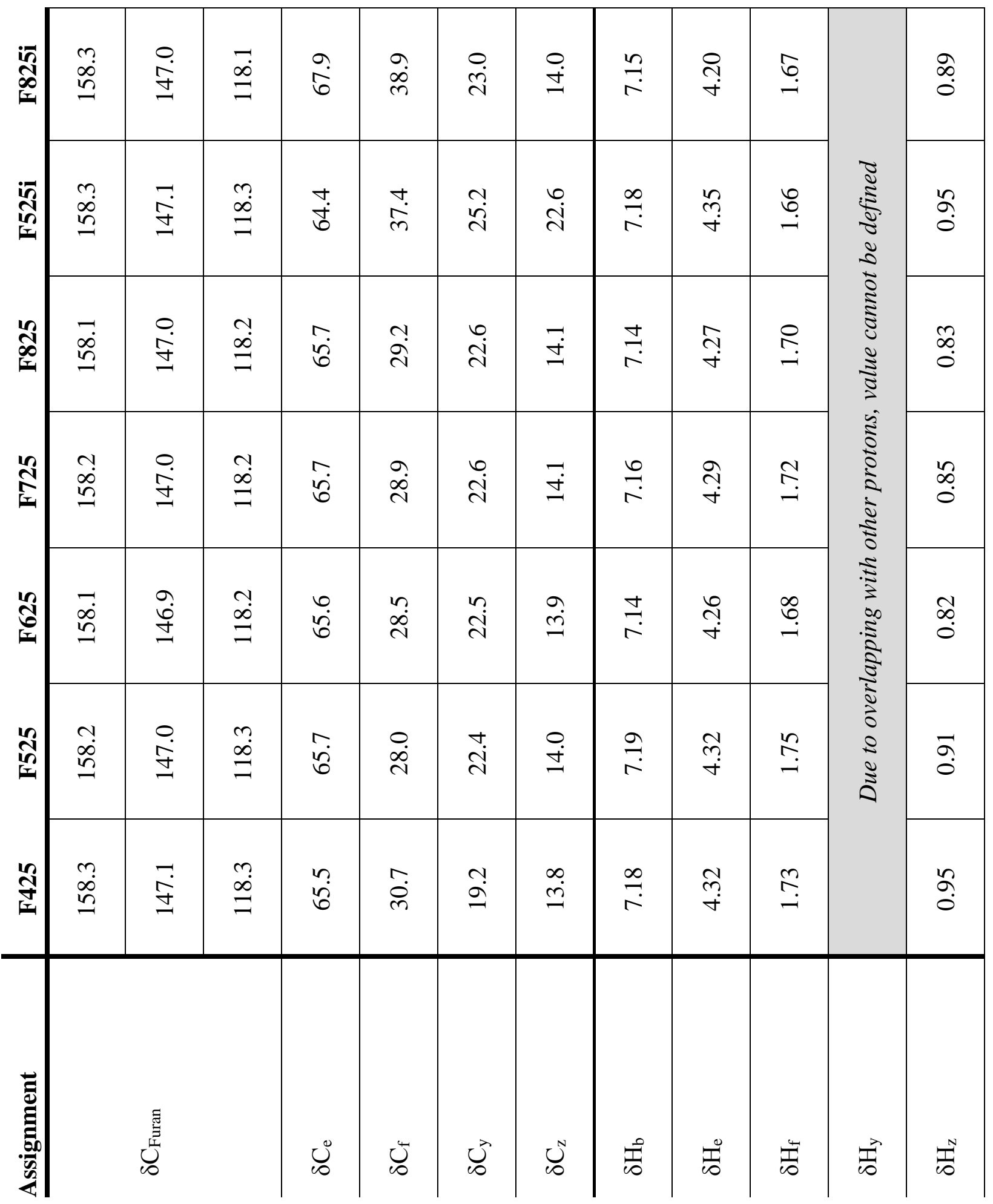


Table S3. Chemical-shift comparison between 2,3-DAFs, only selected is shown (in ppm)

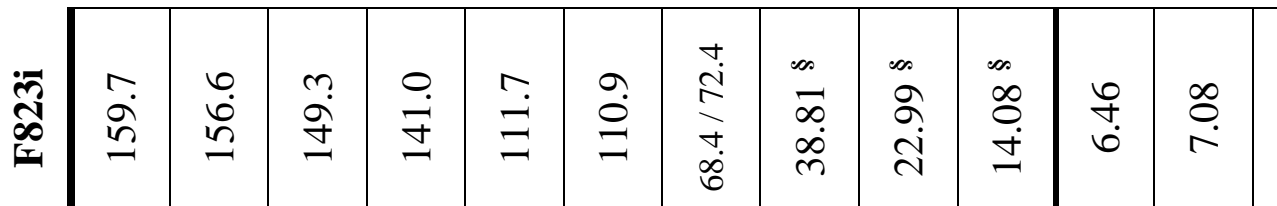

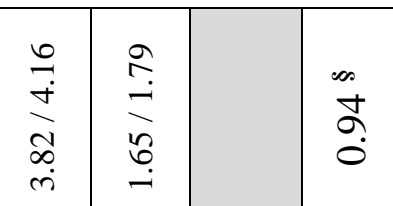

\begin{tabular}{|c|c|c|c|c|c|c|c|c|c|c|c|c|c|}
\hline 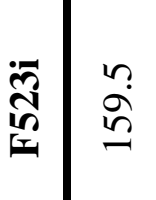 & 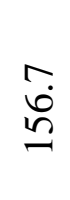 & $\begin{array}{l}\text { o } \\
\stackrel{\infty}{+} \\
\rightarrow\end{array}$ & 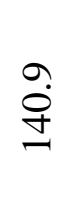 & $\stackrel{\Xi}{\exists}$ & $\stackrel{\exists}{\exists}$ & $\begin{array}{l}m \\
0 \\
0 \\
0 \\
\dot{b} \\
\dot{0}\end{array}$ & $\begin{array}{l}0 \\
\stackrel{0}{d} \\
\text { m. } \\
\text { d. } \\
\text { d. }\end{array}$ & 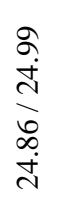 & $\begin{array}{l}\text { Don } \\
\vec{\nabla} \\
\stackrel{N}{N}\end{array}$ & fै & $\vec{r}$ & $\begin{array}{l}\underset{\tilde{m}}{+} \\
\stackrel{+}{\circ} \\
\stackrel{+}{+}\end{array}$ & $\begin{array}{l}\stackrel{+}{\hat{H}} \\
\stackrel{+}{+}\end{array}$ \\
\hline
\end{tabular}

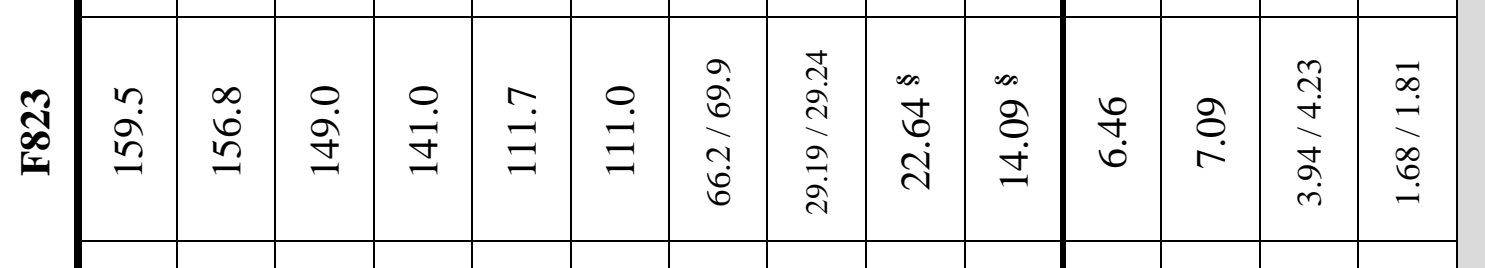

\begin{tabular}{|c|c|c|c|c|c|c|c|c|c|c|c|c|}
\hline 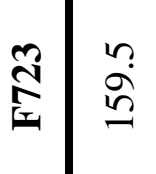 & $\begin{array}{l}\hat{\phi} \\
\stackrel{\leftrightarrow}{~}\end{array}$ & $\begin{array}{l}\text { Oे } \\
\stackrel{\infty}{+}\end{array}$ & $\begin{array}{l}\stackrel{g}{0} \\
\stackrel{+}{-}\end{array}$ & $\stackrel{\Xi}{\exists}$ & $\stackrel{\circ}{\stackrel{\Xi}{\exists}}$ & $\begin{array}{l}\stackrel{0}{0} \\
\dot{0} \\
\stackrel{0}{0} \\
\dot{\theta}\end{array}$ & $\begin{array}{l}\vec{\sigma} \\
\infty \\
0 \\
0 \\
\infty \\
\infty \\
0\end{array}$ & $\begin{array}{l}\infty \\
\stackrel{\infty}{น} \\
\stackrel{2}{N}\end{array}$ & $\begin{array}{l}\infty \\
0 \\
\dot{\mathbb{J}}\end{array}$ & $\begin{array}{l}\mathscr{f} \\
\dot{\varphi}\end{array}$ & $\stackrel{\infty}{\circ}$ & 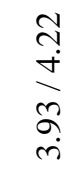 \\
\hline
\end{tabular}

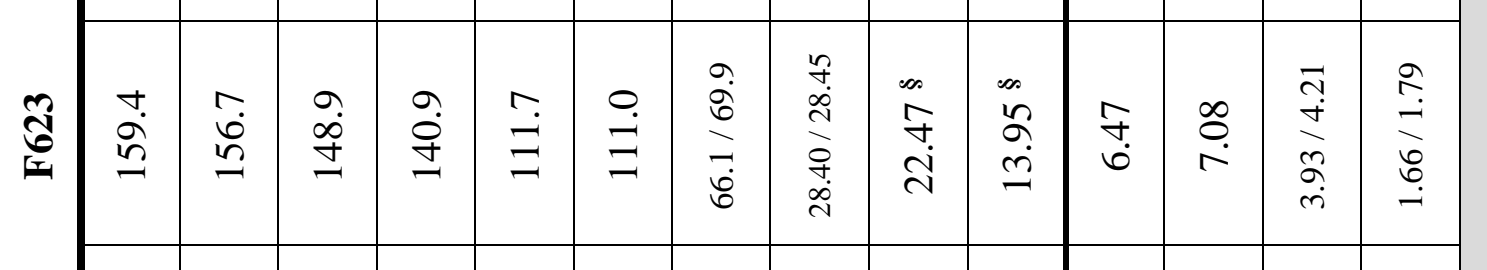

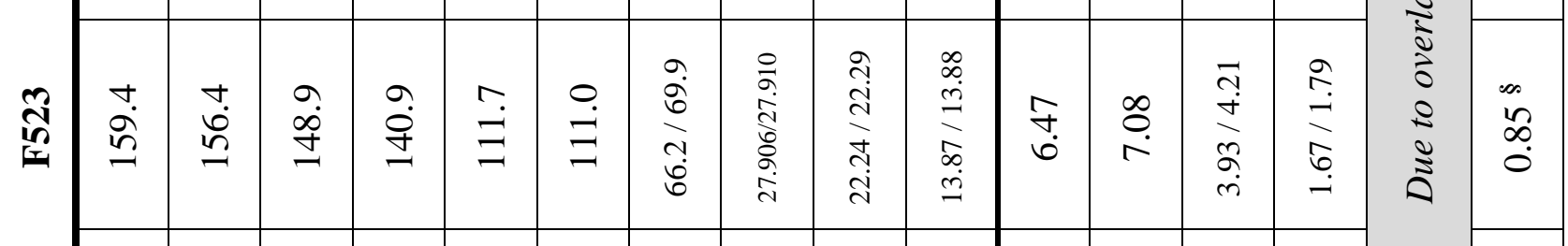

\begin{tabular}{|c|c|c|c|c|c|c|c|c|c|c|c|c|c|c|}
\hline 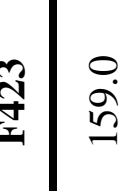 & 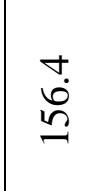 & $\stackrel{\nabla}{+\infty}$ & ma & $\stackrel{亏}{\exists}$ & $\stackrel{-}{\exists}$ & $\begin{array}{l}\text { Jे } \\
\text { Dे } \\
\text { Н. }\end{array}$ & 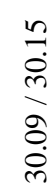 & $\begin{array}{l}\infty \\
0 \\
0 \\
0 \\
\\
\hat{0} \\
\infty \\
0\end{array}$ & 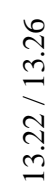 & $\begin{array}{l}\varphi \\
\dot{\varphi}\end{array}$ & बे & 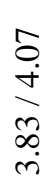 & 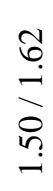 & 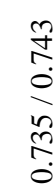 \\
\hline
\end{tabular}

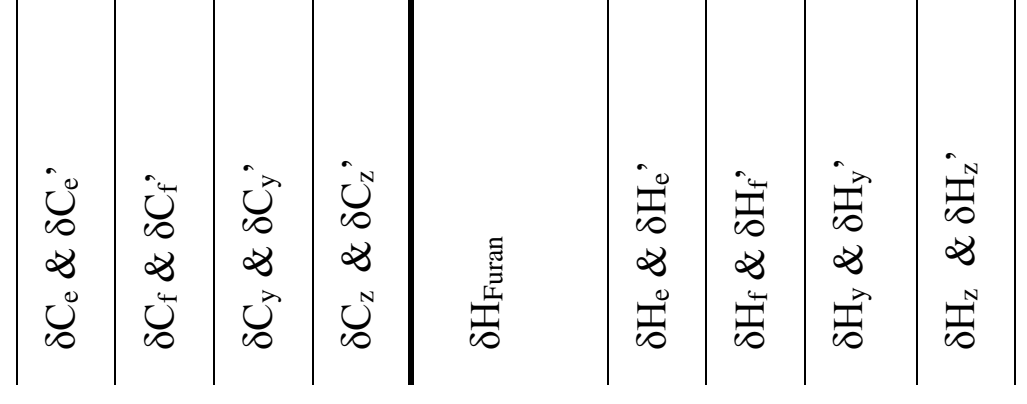




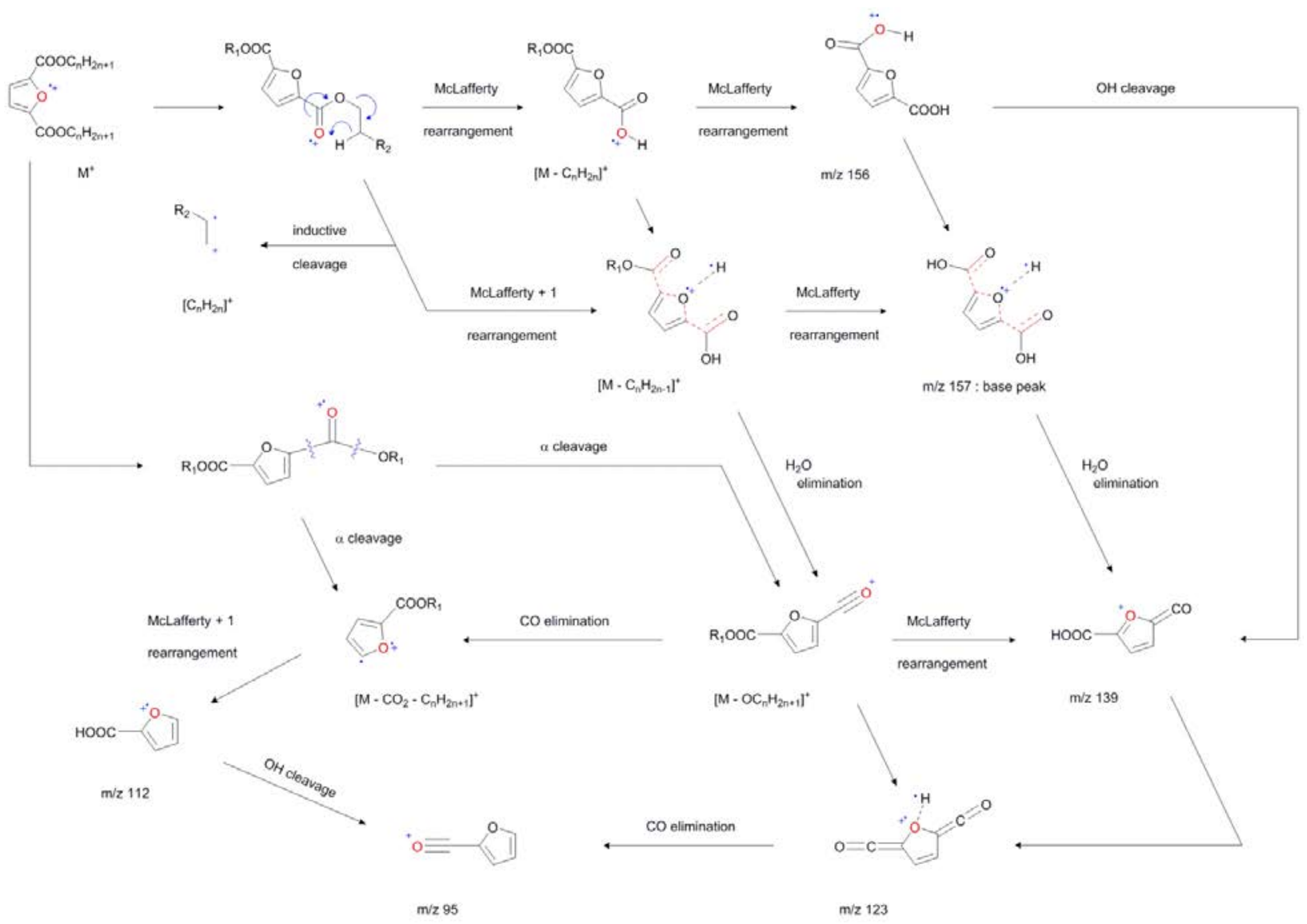

Figure S2. Proposed fragmentation pathway of 2,5-DAFs in mass spectroscopy (EI-MS)

Mass spectra (electron impact, positive mode) provides information about fragmentation reactions of the investigated compounds in the bombardment of electrons. Data from EI-MS, like other spectra, also characterize some structural aspects of the compound.

Figure S2 depicts the fragmentation of 2,5-DAF series step by step. 2,5-DAFs might undergo two McLafferty rearrangements to give out furandicarboxylic acid (2,5-FDCA) as the most intense metastable fragment (m/z 157, basepeak: 100\% intensity), together with an inductive cleavage $\left[\mathrm{C}_{n} \mathrm{H}_{2 n}\right]^{+}$. Then $\mathrm{m} / \mathrm{z} 139$ was created (the $2^{\text {nd }}$ most intense peak). The molecular ions $\mathrm{M}^{+}$were generally observable. From molecular ions, other recognizable fragments $\left[\mathrm{M}-\mathrm{OC}_{n} \mathrm{H}_{2 n+1}\right]^{+}$and 
$\left[\mathrm{M}-\mathrm{CO}_{2}-\mathrm{C}_{n} \mathrm{H}_{2 \mathrm{n}+1}\right]^{+}$were formed, owing to $\alpha$-cleavage of carbonyl group. Those two fragments are signature for any ester compounds.

For the series of 2,3-DAFs, the complications of fragmentation very much arise possibly regarding a specific conformation or the ortho effect(s) caused by neighboring groups. Firstly, it could be found that $\mathrm{m} / \mathrm{z} 156$ and/or m/z 157 (representing 2,3-FDCA core; formed through McLaffterty rearrangements), were not basepeaks, but still remarkably intense. Secondly, the existence of a unique metastable fragment $\left[\mathrm{M}-\mathrm{C}_{\mathrm{n}-1} \mathrm{H}_{2 \mathrm{n}-1}\right]^{+}$and the massive production of many high-intensity hydrocarbon fragments ( $\mathrm{m} / \mathrm{z} 41,43,55,57$ etc.) insinuated the fact that the fragmentation chain reactions were kicked off firstly by reducing length of an aliphatic chain. Lastly but the most importantly, the appearances of several even-numbered mass-to-charge ratios (in particular: $\mathrm{m} / \mathrm{z}$ 82, m/z 128 etc.), were barely absent from 2,5-DAF spectra. Provided in compounds containing only $\mathrm{C}, \mathrm{H}$, O elements, fragments resulting from a single cleavage always appear at an odd $\mathrm{m} / \mathrm{z}$, therefore there might have other rearrangement(s) beside McLafferty rearrangement to produce even $\mathrm{m} / \mathrm{z}$ peaks in 2,3-DAF spectra. The proposal for fragmentation pathway of 2,3-DAF series was depicted in Figure S3, as follow. 


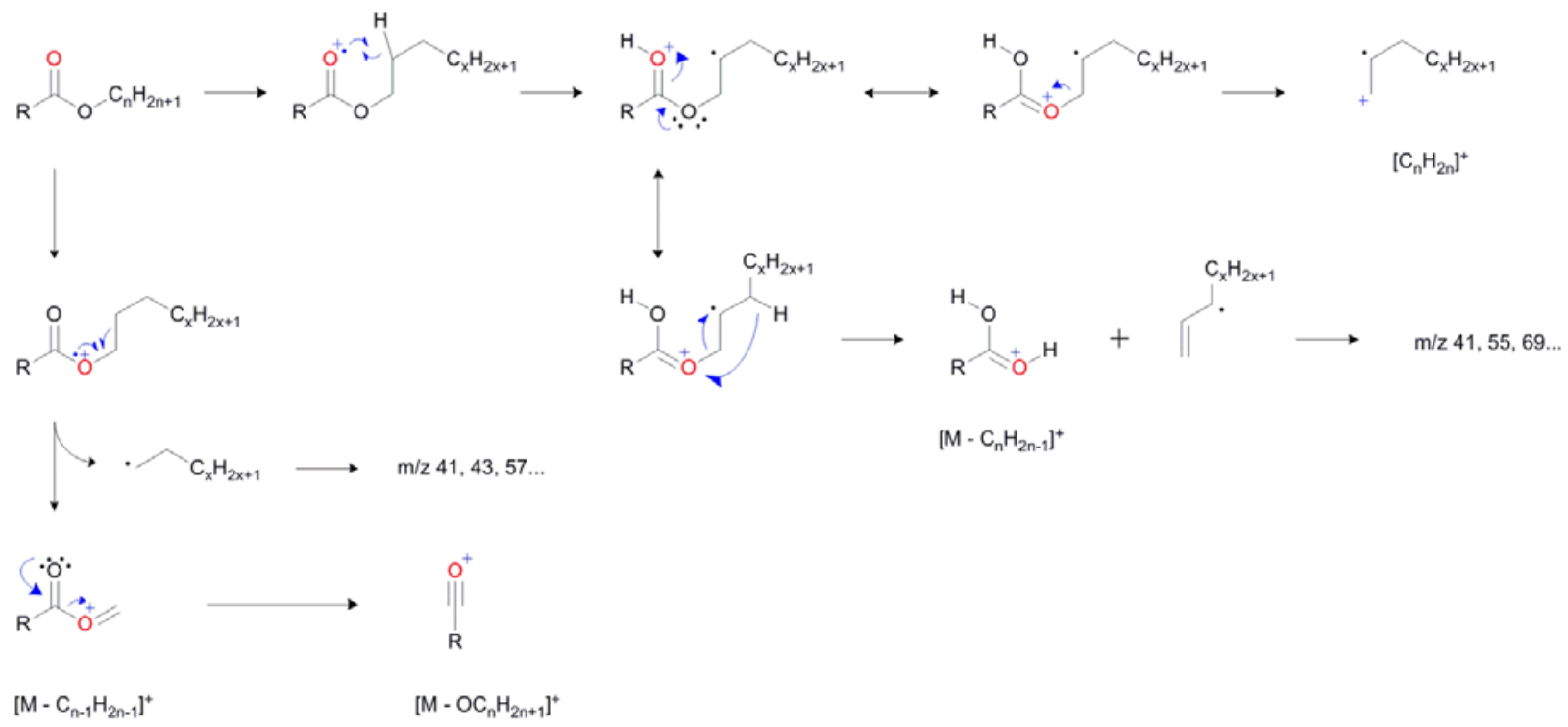

Figure S3. Proposed initial events happened in an aliphatic branch of 2,3-DAF in EI-MS
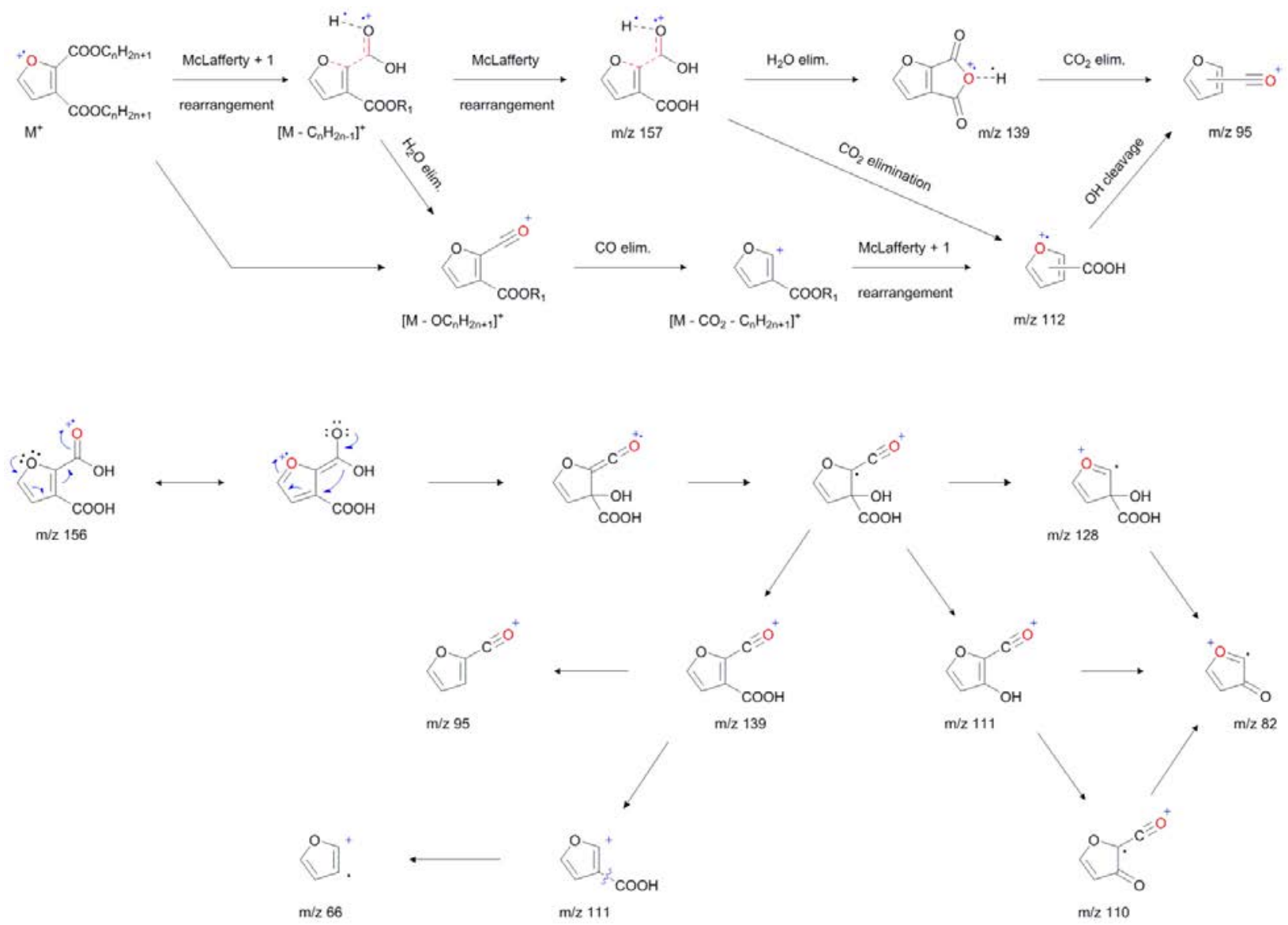

Figure S4. Proposed fragmentation pathway of 2,3-DAFs in mass spectroscopy (EI-MS) 
During reaction, alkyl 2-furoates were also formed and detected via GC-MS. tigation. As can be seen, the fragmentation reactions of 2-furoates were uncomplicated and could be well interpreted. To sum up, it was only about cleavage and no other rearrangement than McLafferty rearrangement.

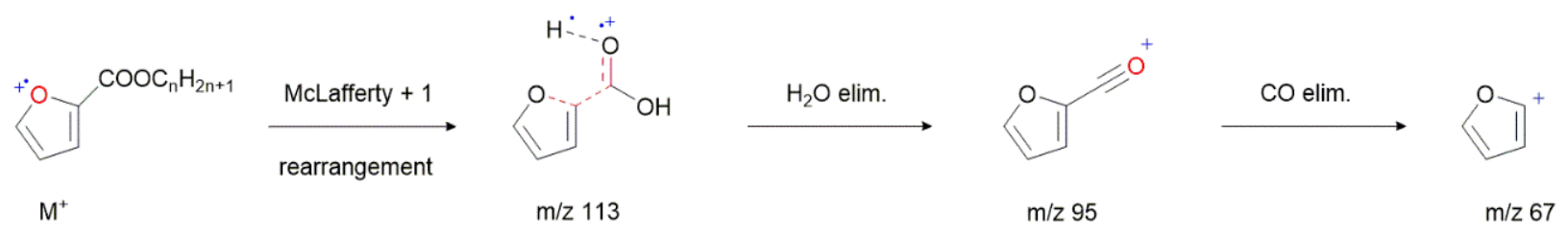

Figure S5. Fragmentation pathway of alkyl 2-furoate in mass spectroscopy (EI-MS) 
Table S4. Fragmentation-pattern comparison between 2,5-DAF compounds, in m/z

\begin{tabular}{|c|c|c|c|c|c|c|c|c|c|c|}
\hline 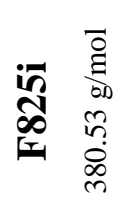 & 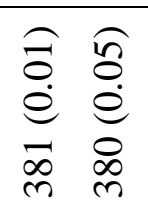 & 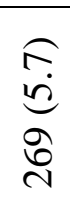 & 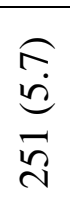 & 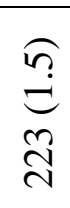 & 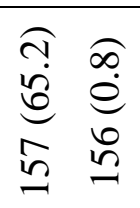 & 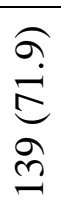 & 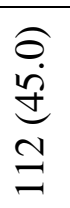 & $\begin{array}{l}\hat{\alpha} \\
\stackrel{0}{0} \\
\swarrow \alpha\end{array}$ & 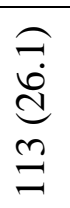 & 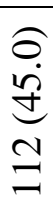 \\
\hline 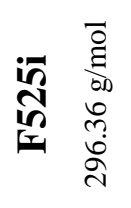 & 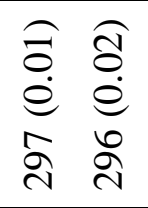 & 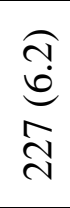 & 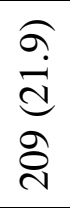 & 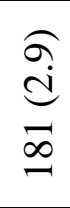 & 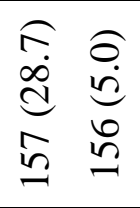 & 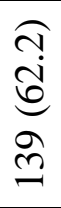 & 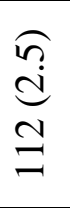 & 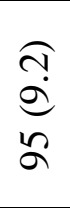 & $\frac{\stackrel{\overbrace{}}{\vec{J}}}{\underset{\Sigma}{ \pm}}$ & $\begin{array}{l}\stackrel{8}{8} \\
\stackrel{0}{\circ}\end{array}$ \\
\hline 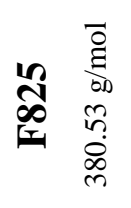 & 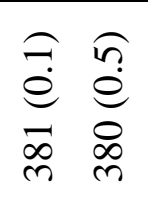 & 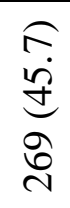 & 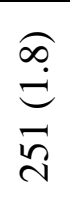 & 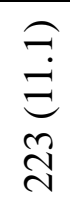 & 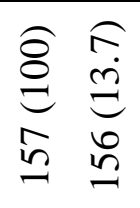 & 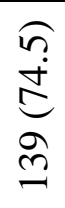 & 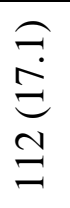 & 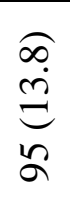 & 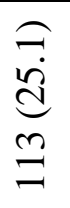 & 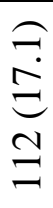 \\
\hline 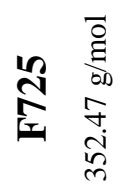 & 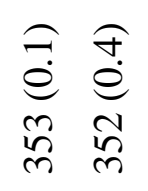 & 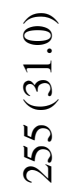 & $\begin{array}{l}\underset{n}{\infty} \\
\stackrel{\hat{N}}{\hat{N}}\end{array}$ & 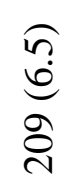 & 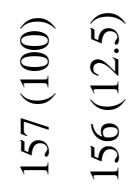 & 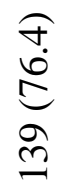 & 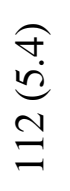 & 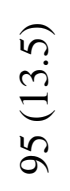 & 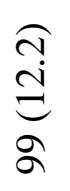 & 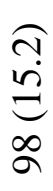 \\
\hline 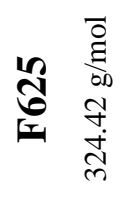 & 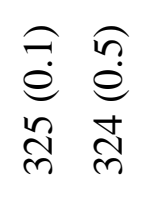 & $\begin{array}{l}6 \\
\dot{d} \\
\stackrel{d}{d} \\
\vec{d}\end{array}$ & 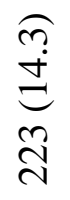 & 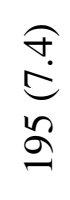 & 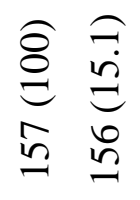 & 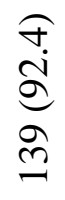 & 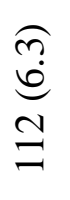 & 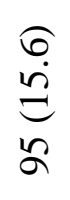 & 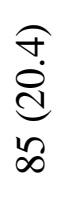 & 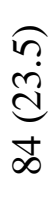 \\
\hline 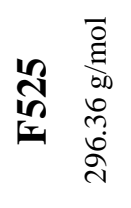 & 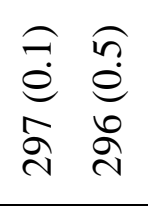 & 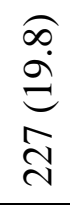 & 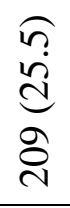 & 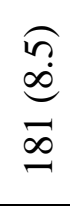 & 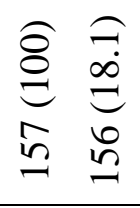 & 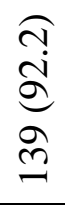 & 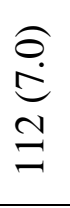 & $\underbrace{\stackrel{\infty}{ \pm}}_{\mathscr{\sigma}}$ & $\underbrace{\stackrel{\sigma}{\oplus}}_{\overrightarrow{1}}$ & 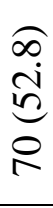 \\
\hline 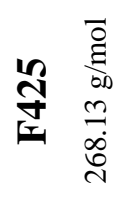 & 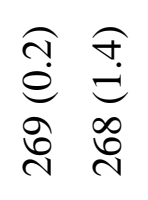 & 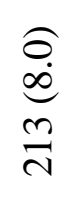 & 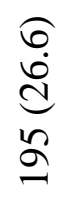 & $\begin{array}{l}\sigma \\
\sigma \\
\hat{\sigma} \\
-1\end{array}$ & 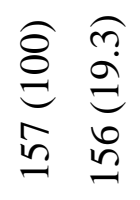 & 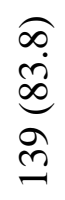 & $\begin{array}{l}\underset{\Xi}{\Xi} \\
\stackrel{7}{\exists}\end{array}$ & $\underbrace{\stackrel{\sigma}{ٍ}}_{\stackrel{\sigma}{\sigma}}$ & 点 & 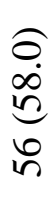 \\
\hline 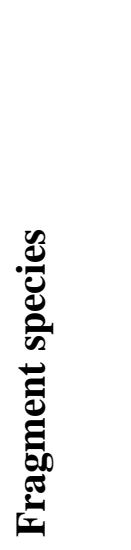 & 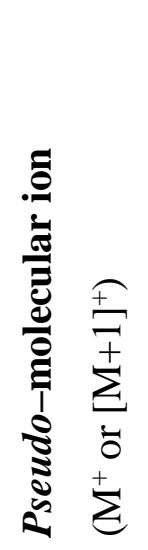 & 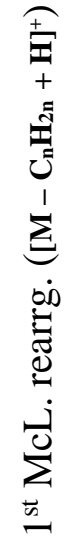 & 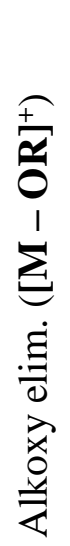 & 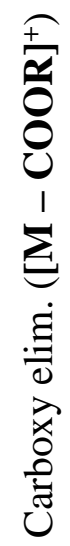 & 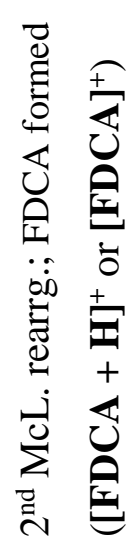 & 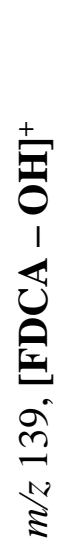 & 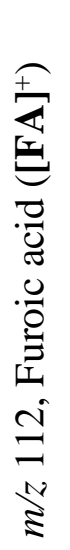 & \begin{tabular}{l}
$\frac{+}{5}$ \\
0 \\
1 \\
$\vdots$ \\
\multirow{1}{1}{} \\
ñ \\
N \\
है
\end{tabular} & 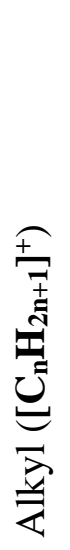 & 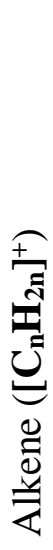 \\
\hline
\end{tabular}


Table S5. Fragmentation-pattern comparison between 2,3-DAF compounds, in m/z

\begin{tabular}{|c|c|c|c|c|c|c|c|c|c|c|c|c|}
\hline 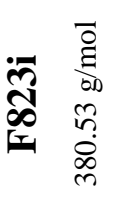 & 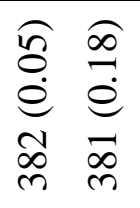 & 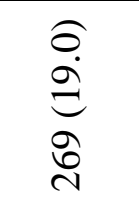 & 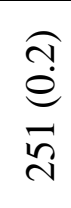 & 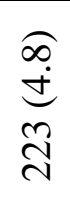 & 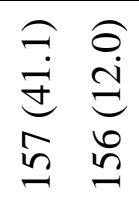 & 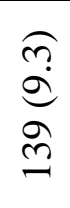 & 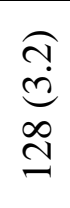 & $\begin{array}{l}\underset{7}{\theta} \\
\dot{\theta} \\
\exists \\
\exists\end{array}$ & 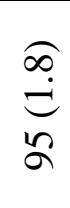 & 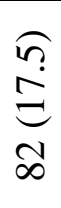 & 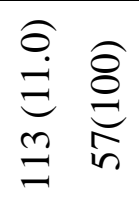 & 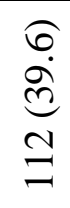 \\
\hline $\begin{array}{l}\overline{0} \\
\text { हू } \\
0 \\
0 \\
0\end{array}$ & 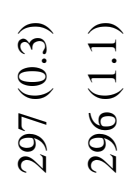 & 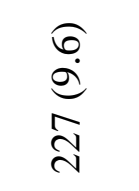 & $\begin{array}{l}\hat{a} \\
\stackrel{e}{2} \\
\stackrel{\partial}{2}\end{array}$ & $\underset{\substack{0 \\
-1 \\
-1}}{\stackrel{0}{+}}$ & 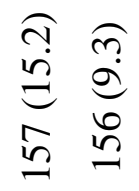 & 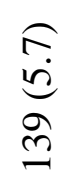 & 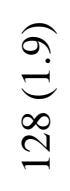 & $\begin{array}{l}\underset{\infty}{\infty} \\
\exists \\
\exists\end{array}$ & 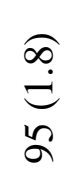 & $\begin{array}{l}\underset{f}{\stackrel{0}{\Xi}} \\
\underset{\infty}{\infty}\end{array}$ & 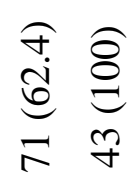 & 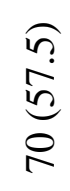 \\
\hline 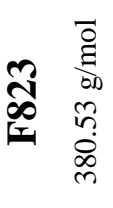 & 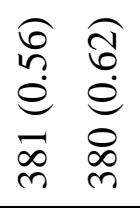 & $\begin{array}{l}\text { ్. } \\
\text { ले } \\
\text { के } \\
\stackrel{2}{2}\end{array}$ & $\begin{array}{l}\text { ô } \\
\dot{e} \\
-1 \\
\stackrel{D}{2}\end{array}$ & 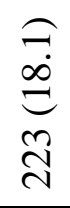 & 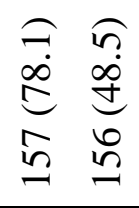 & 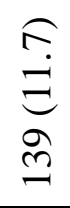 & 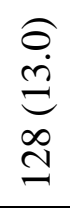 & $\begin{array}{l}\overparen{m} \\
\infty \\
\cdots \\
\exists\end{array}$ & 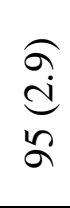 & 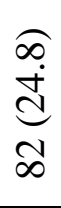 & 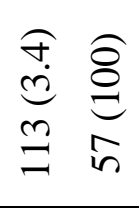 & 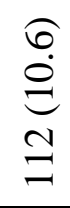 \\
\hline $\begin{array}{l}\overrightarrow{0} \\
\vec{E} \\
\text { of } \\
\hat{f}\end{array}$ & 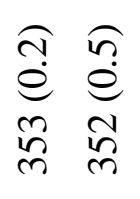 & 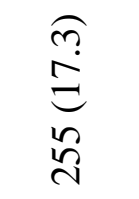 & 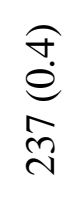 & 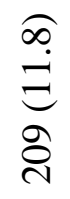 & 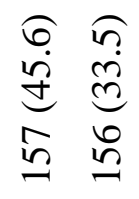 & 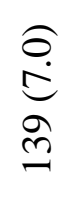 & 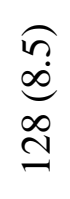 & 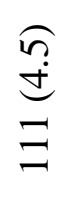 & 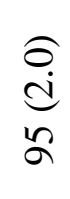 & 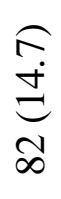 & 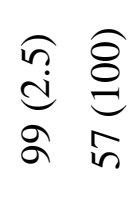 & $\begin{array}{l}\sigma \\
\hat{\theta} \\
\infty \\
\infty\end{array}$ \\
\hline $\bar{g}$ & 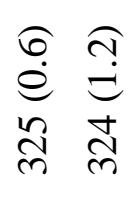 & $\begin{array}{l}\underset{m}{g} \\
\underset{\vec{g}}{\vec{d}}\end{array}$ & 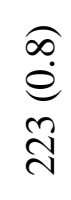 & $\begin{array}{l}\underset{\infty}{\dot{d}} \\
\stackrel{d}{d} \\
\stackrel{\mathscr{D}}{\sigma}\end{array}$ & 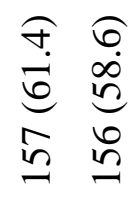 & $\begin{array}{l}\stackrel{\dot{O}}{\sigma} \\
\stackrel{\text { m}}{\sim}\end{array}$ & 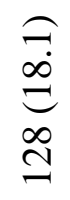 & 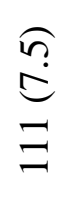 & $\begin{array}{l}\overparen{0} \\
\stackrel{0}{\mathscr{n}}\end{array}$ & 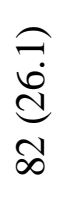 & 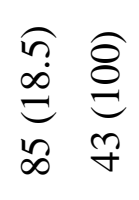 & $\underset{⿱ 亠 凶}{\stackrel{\sigma}{\Xi}}$ \\
\hline $\bar{g}$ & 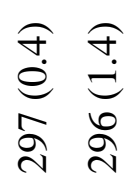 & $\begin{array}{l}\widehat{̃} \\
\infty \\
\widehat{N}\end{array}$ & 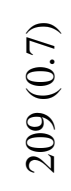 & 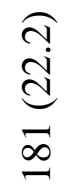 & 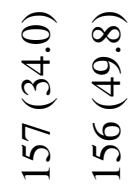 & 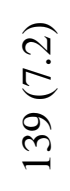 & 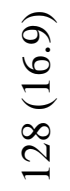 & 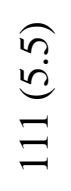 & 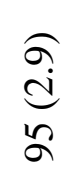 & 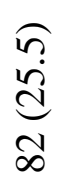 & 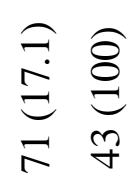 & 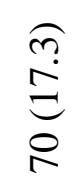 \\
\hline $\begin{array}{l}\overrightarrow{\mathrm{g}} \\
\stackrel{\mathrm{g}}{\mathrm{m}} \\
\stackrel{m}{\mathrm{n}}\end{array}$ & 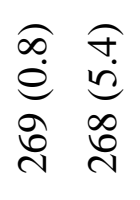 & 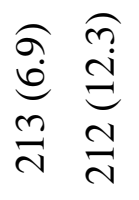 & 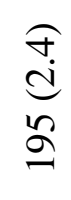 & 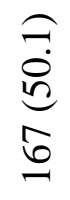 & 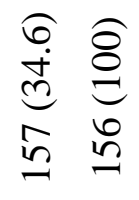 & 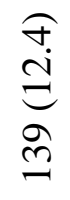 & 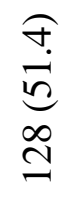 & 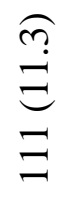 & $\begin{array}{l}\stackrel{0}{0} \\
\stackrel{0}{0}\end{array}$ & 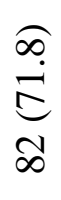 & $\underset{\text { î }}{\stackrel{\overbrace{}}{\hat{E}}}$ & 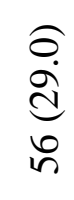 \\
\hline & 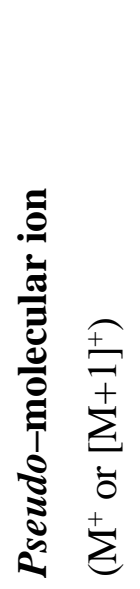 & 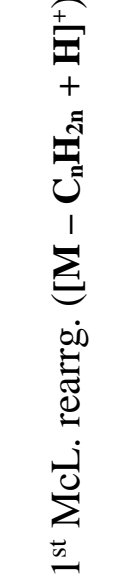 & 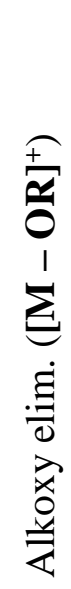 & 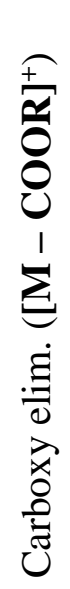 & 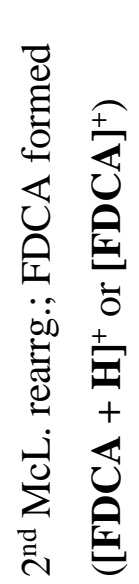 & 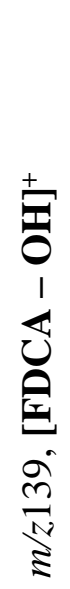 & $\begin{array}{l}\stackrel{\infty}{N} \\
\stackrel{N}{N} \\
\text { I }\end{array}$ & 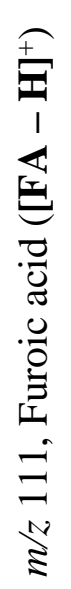 & 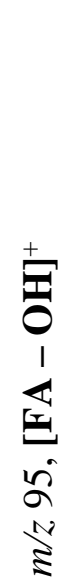 & $\begin{array}{l}\infty \\
\text { है }\end{array}$ & 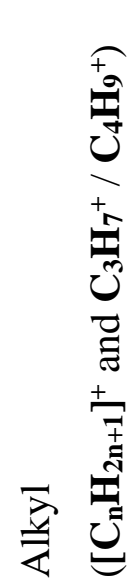 & 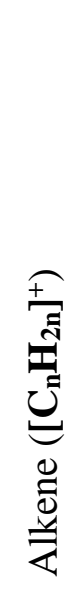 \\
\hline
\end{tabular}


Table S6. Infrared absorption comparison between 2,5-DAF compounds, in wavenumber $\left(\mathrm{cm}^{-1}\right)$

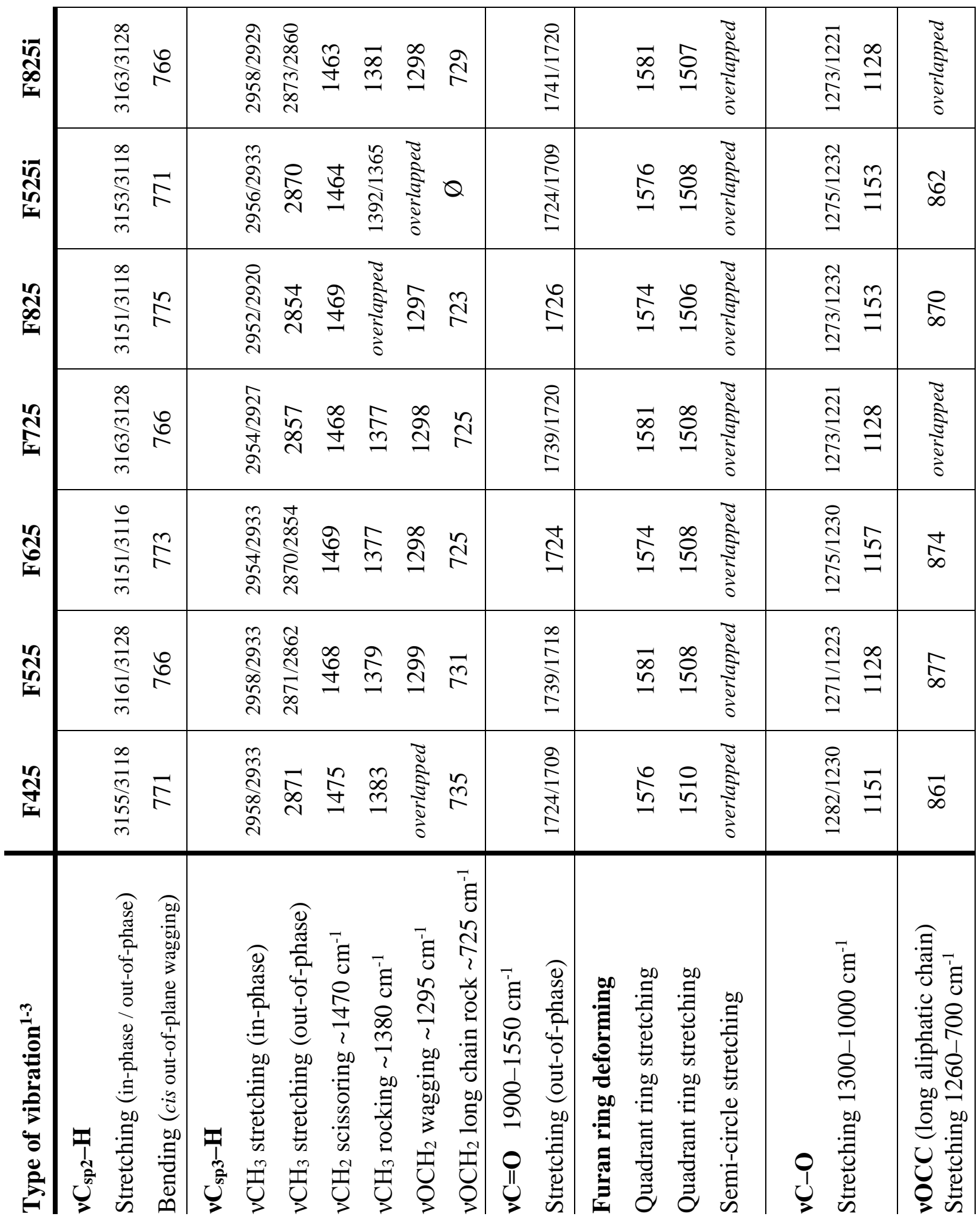


Table S7. Infrared absorption comparison between 2,3-DAF compounds, in wavenumber $\left(\mathrm{cm}^{-1}\right)$

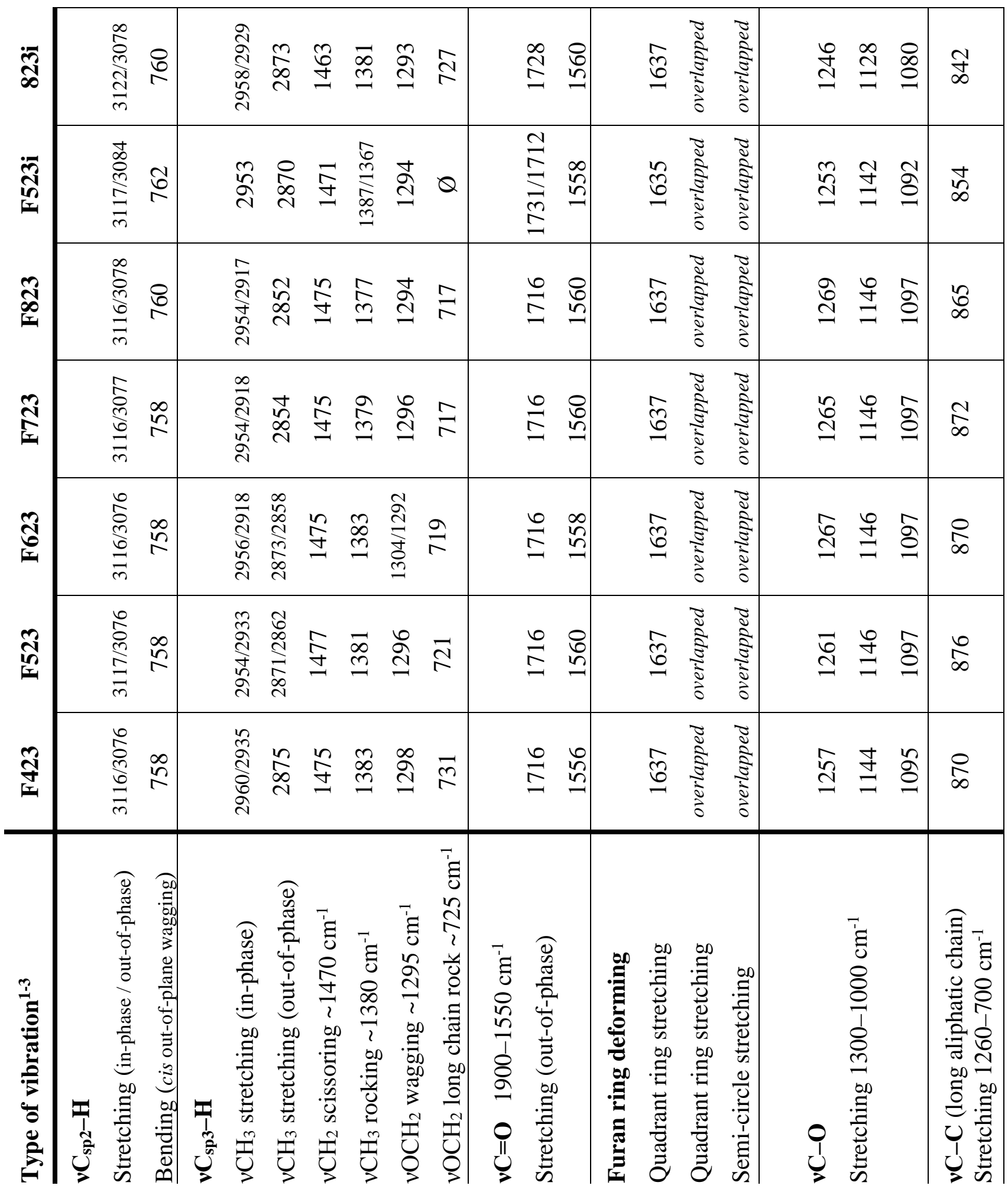


1. Dialkyl furan-2,5-dicarboxylates (2,5-DAFs)

A - DIBUTYL FURAN-2,5-DICARBOXYLATE (2,5-DBF)

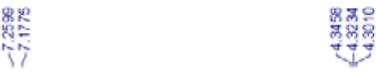
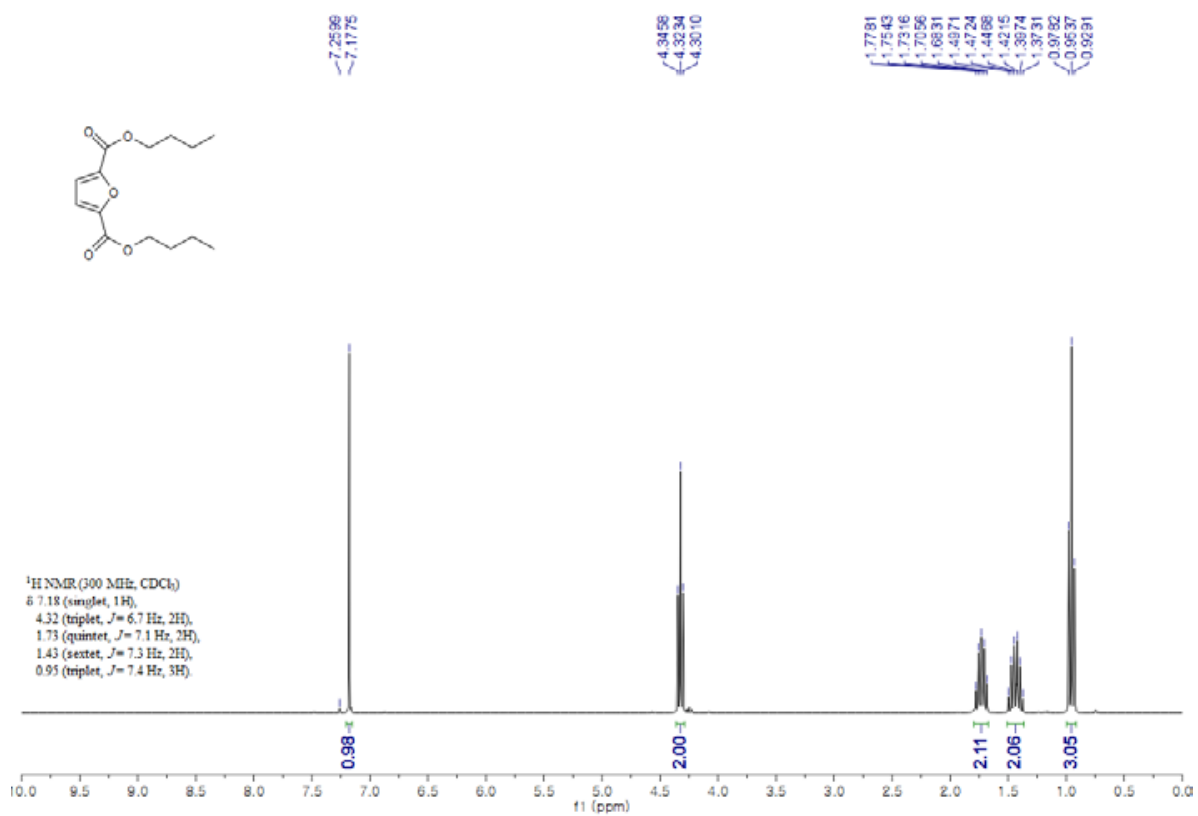

Figure S6. ${ }^{1} \mathrm{H}-\mathrm{NMR}$ spectrum of dibutyl furan-2,5-dicarboxylate (2,5-DBF)

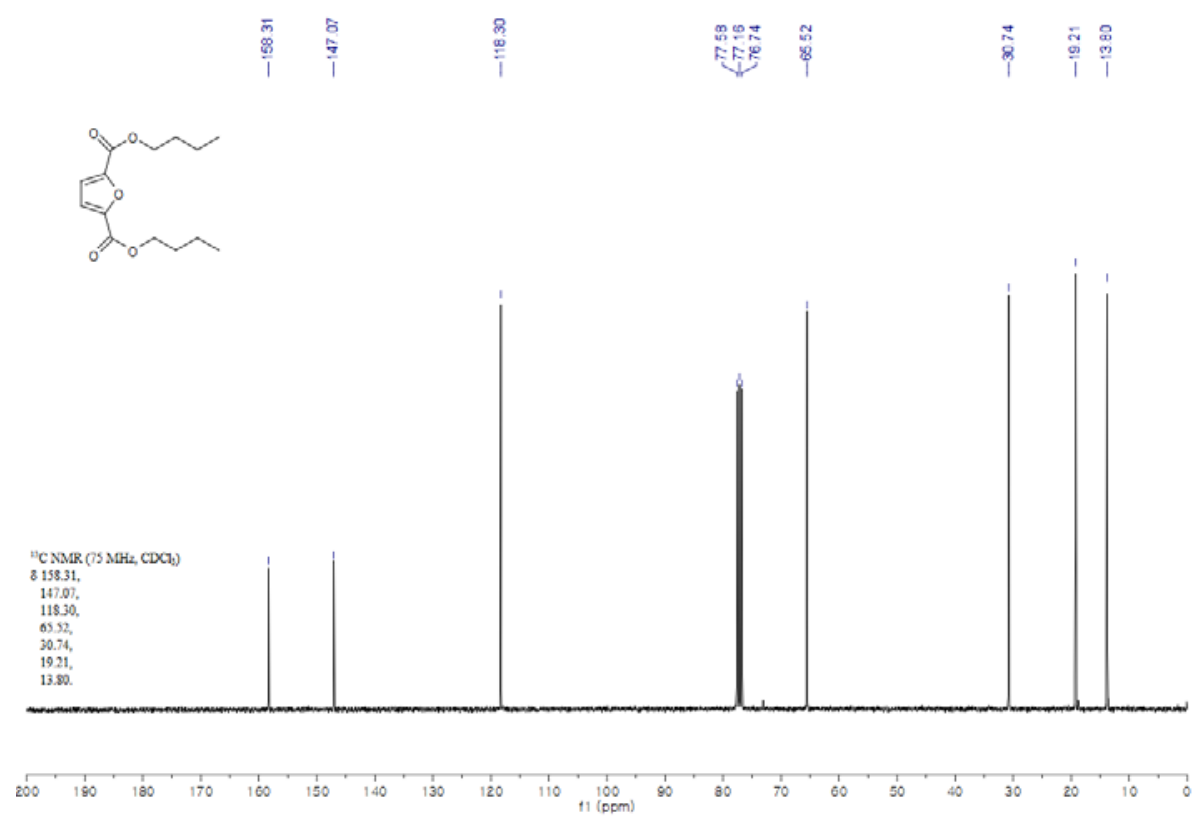

Figure S7. ${ }^{13} \mathrm{C}-\mathrm{NMR}$ spectrum of dibutyl furan-2,5-dicarboxylate (2,5-DBF) 

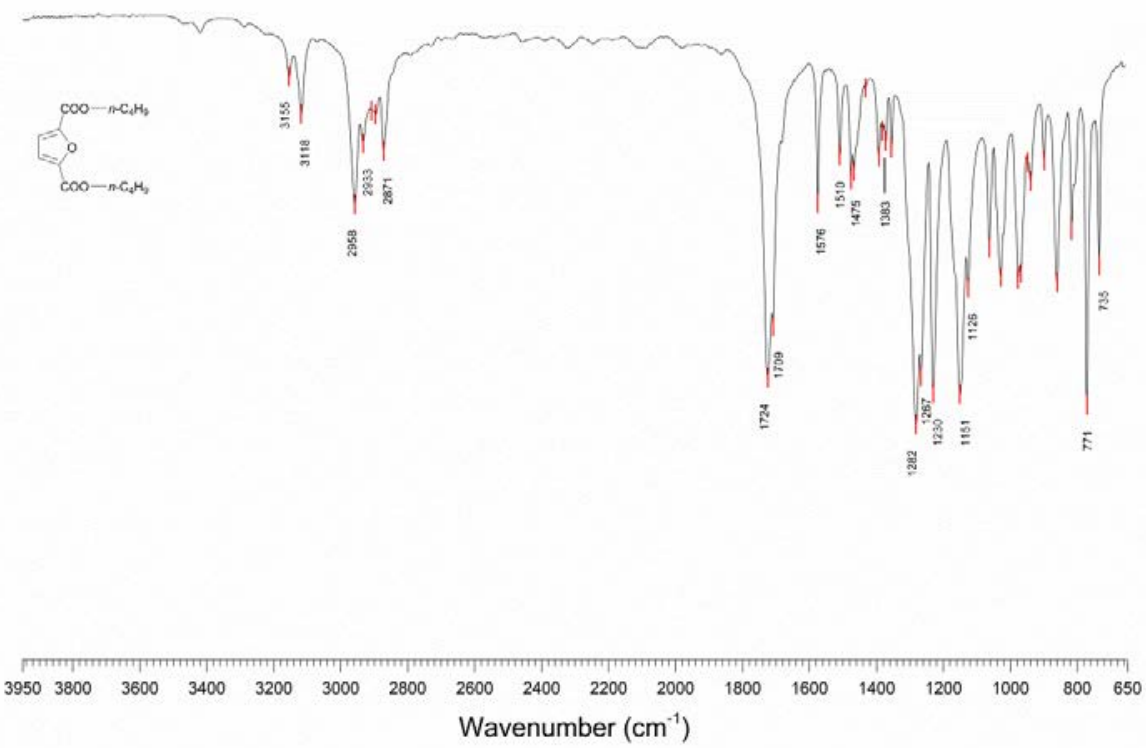

Figure S8. Infrared spectrum of dibutyl furan-2,5-dicarboxylate (2,5-DBF)

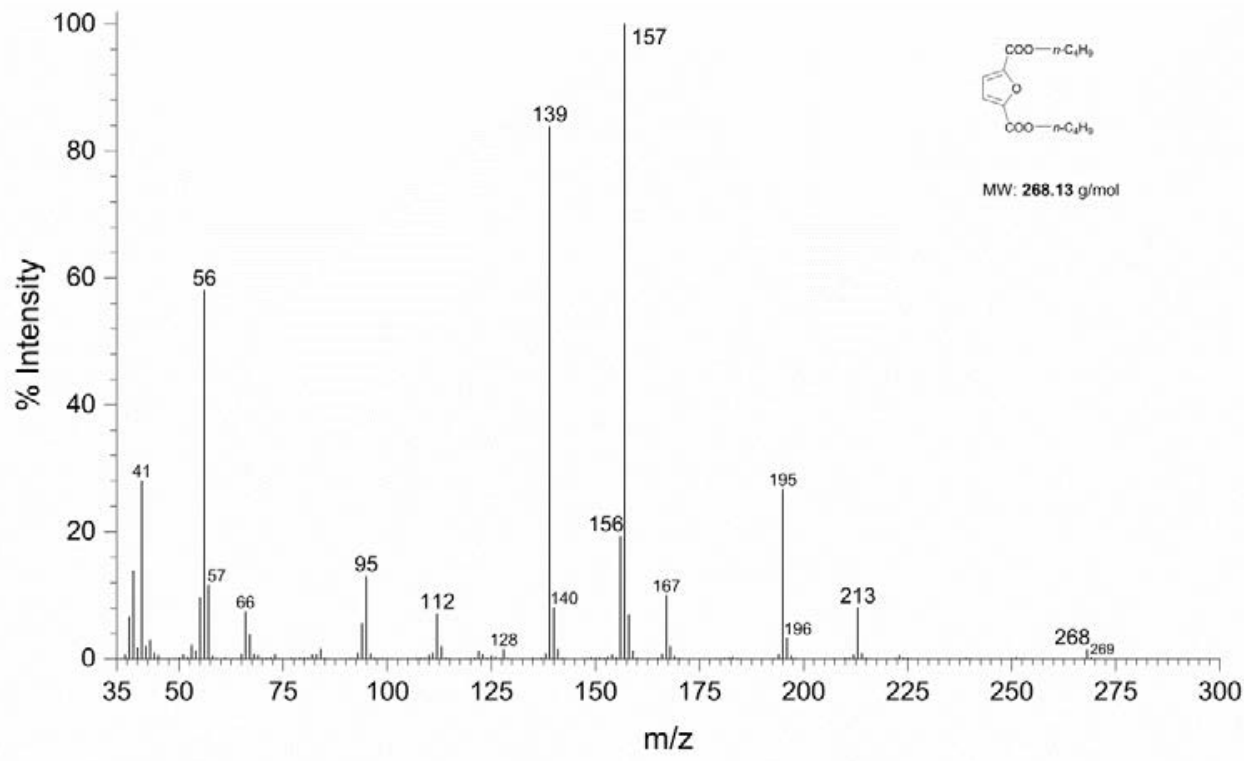

Figure S9. Mass spectrum of dibutyl furan-2,5-dicarboxylate (2,5-DBF) 
B - DIPENTYL FURAN-2,5-DICARBOXYLATE

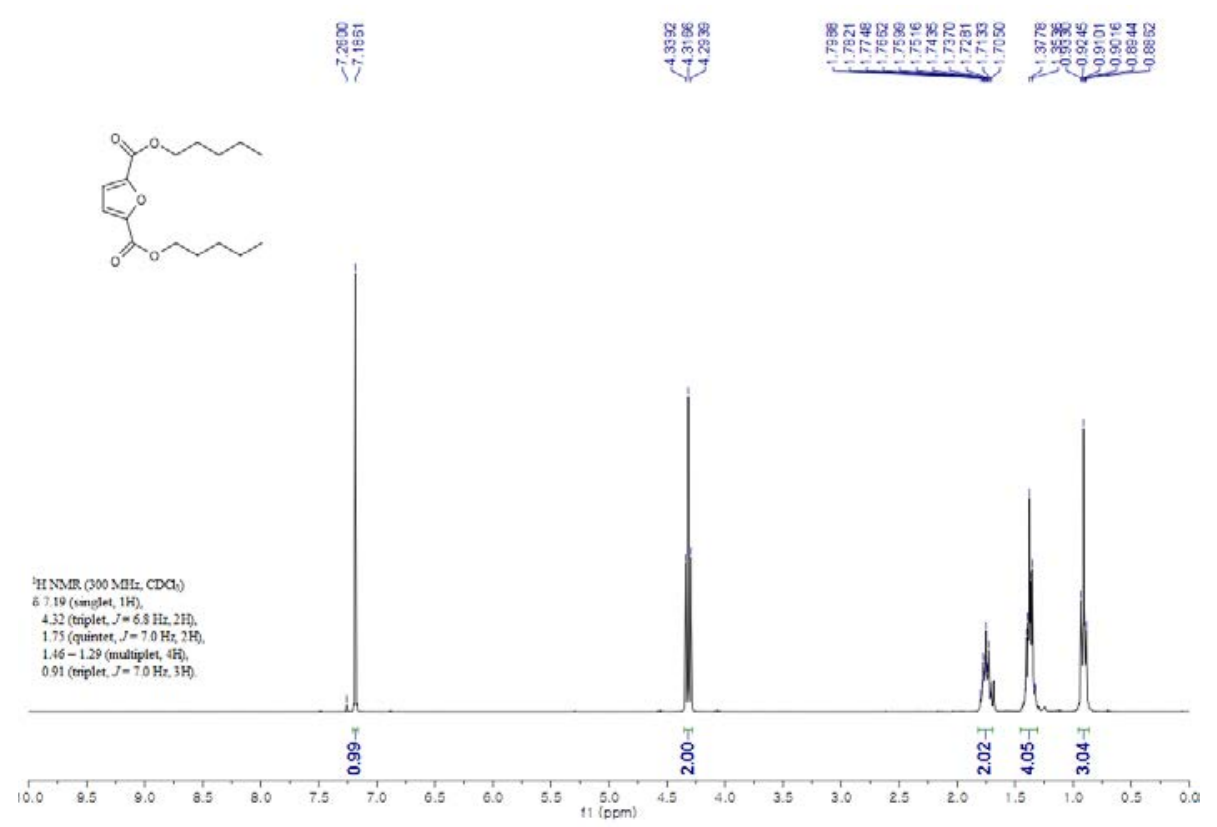

Figure S10. ${ }^{1} \mathrm{H}$-NMR spectrum of dipentyl furan-2,5-dicarboxylate

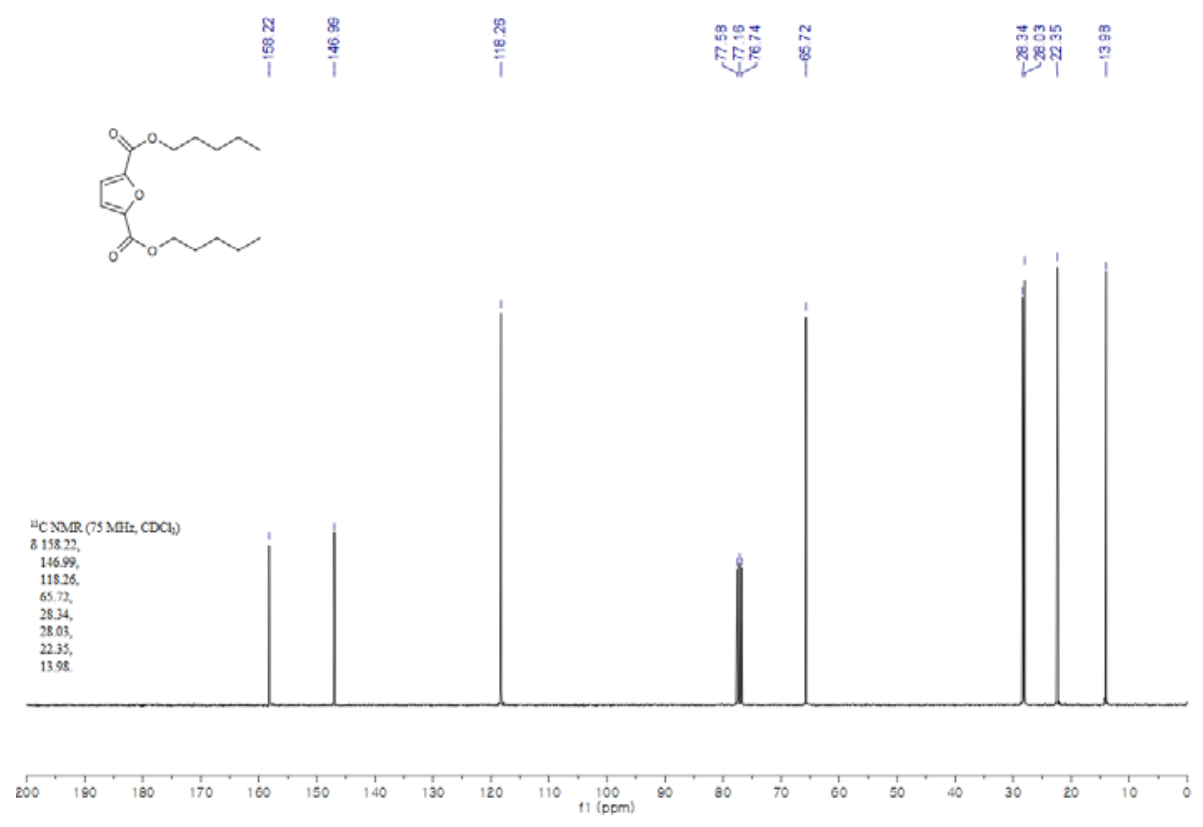

Figure S11. ${ }^{13} \mathrm{C}$-NMR spectrum of dipentyl furan-2,5-dicarboxylate 


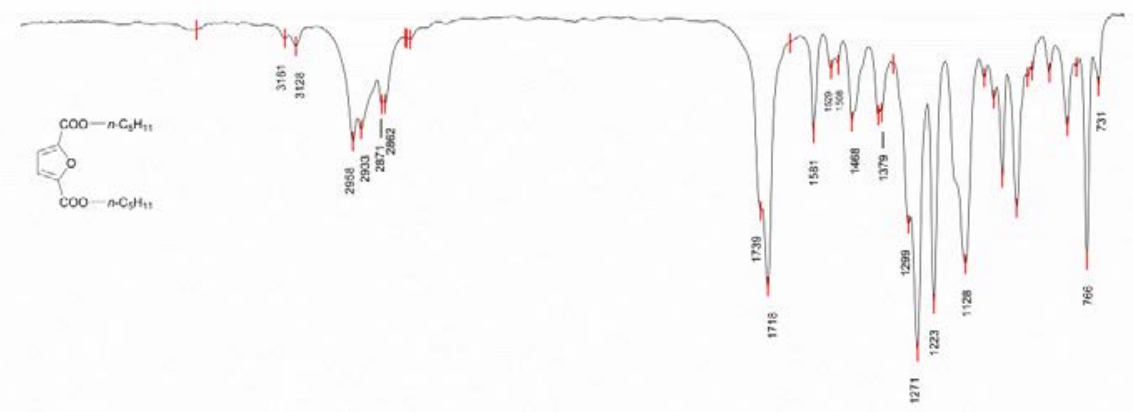

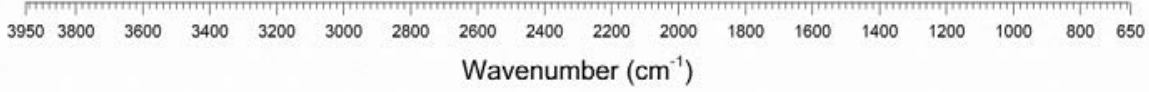

Figure S12. Infrared spectrum of dipentyl furan-2,5-dicarboxylate

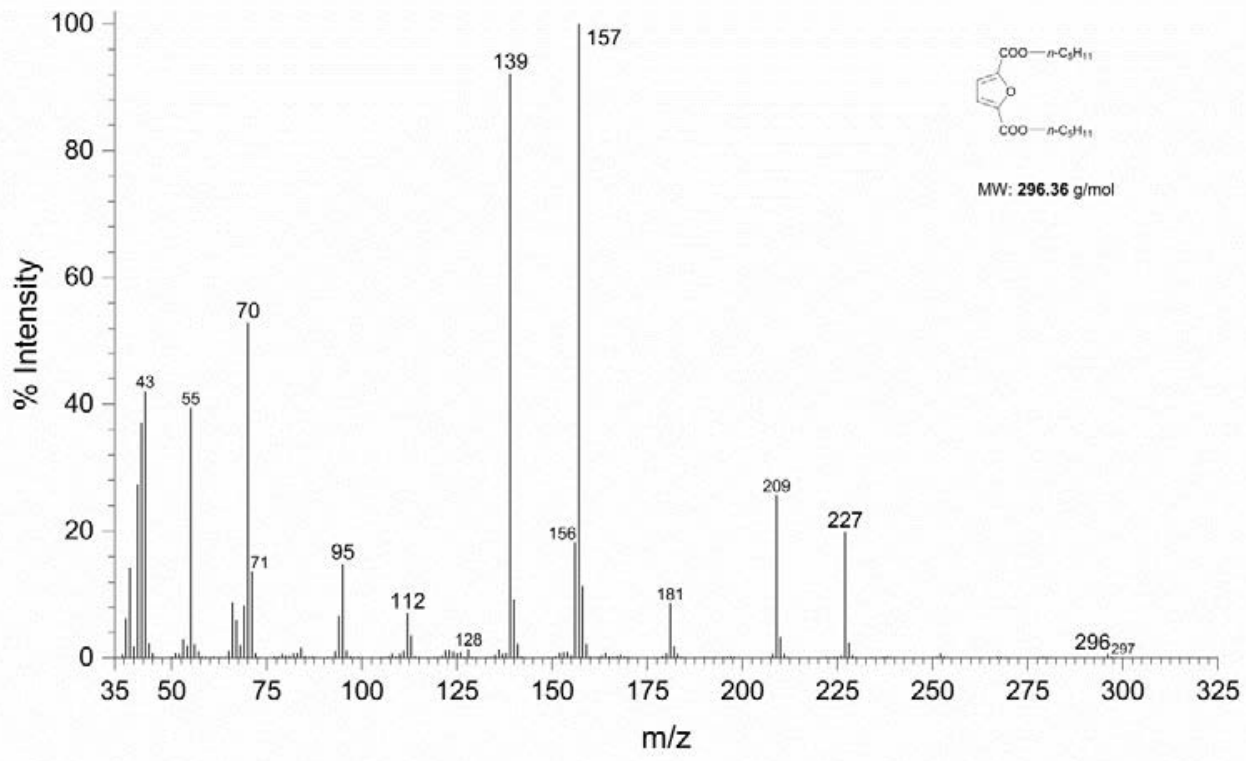

Figure S13. Mass spectrum of dipentyl furan-2,5-dicarboxylate 


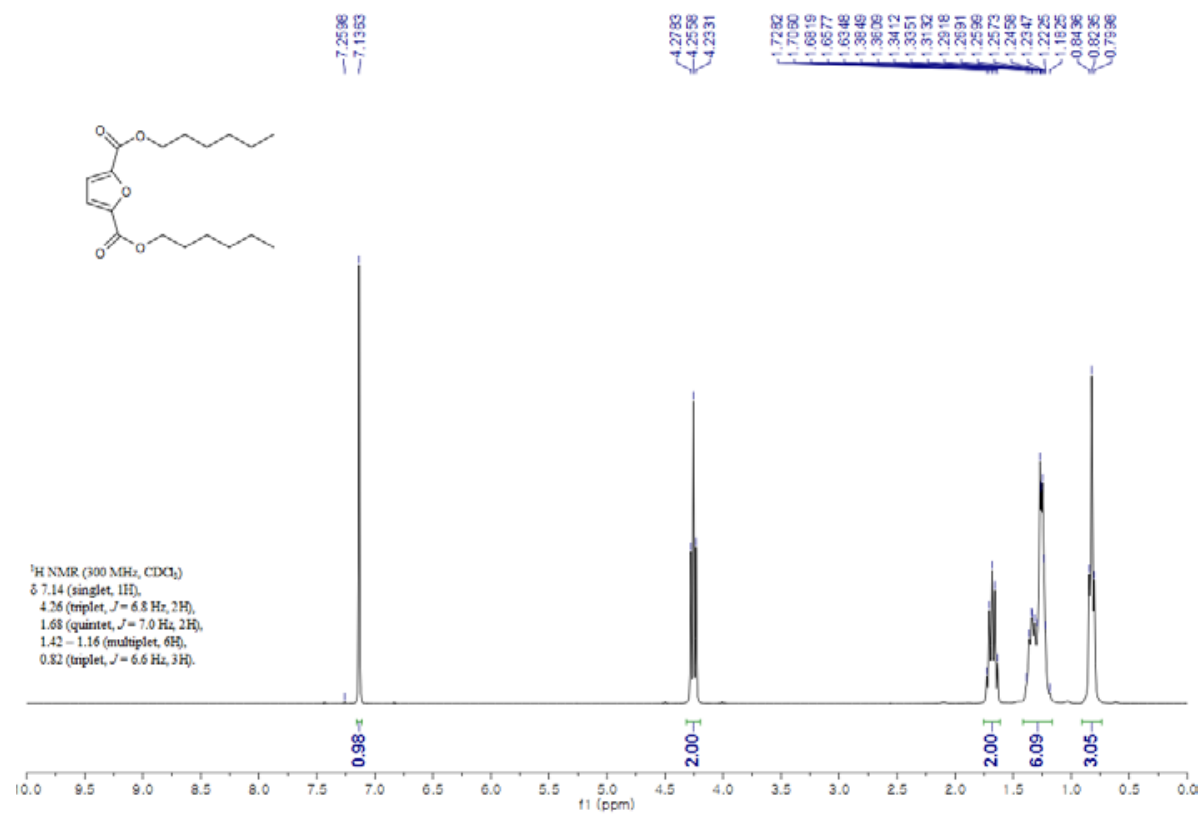

Figure S14. ${ }^{1} \mathrm{H}-\mathrm{NMR}$ spectrum of dihexyl furan-2,5-dicarboxylate

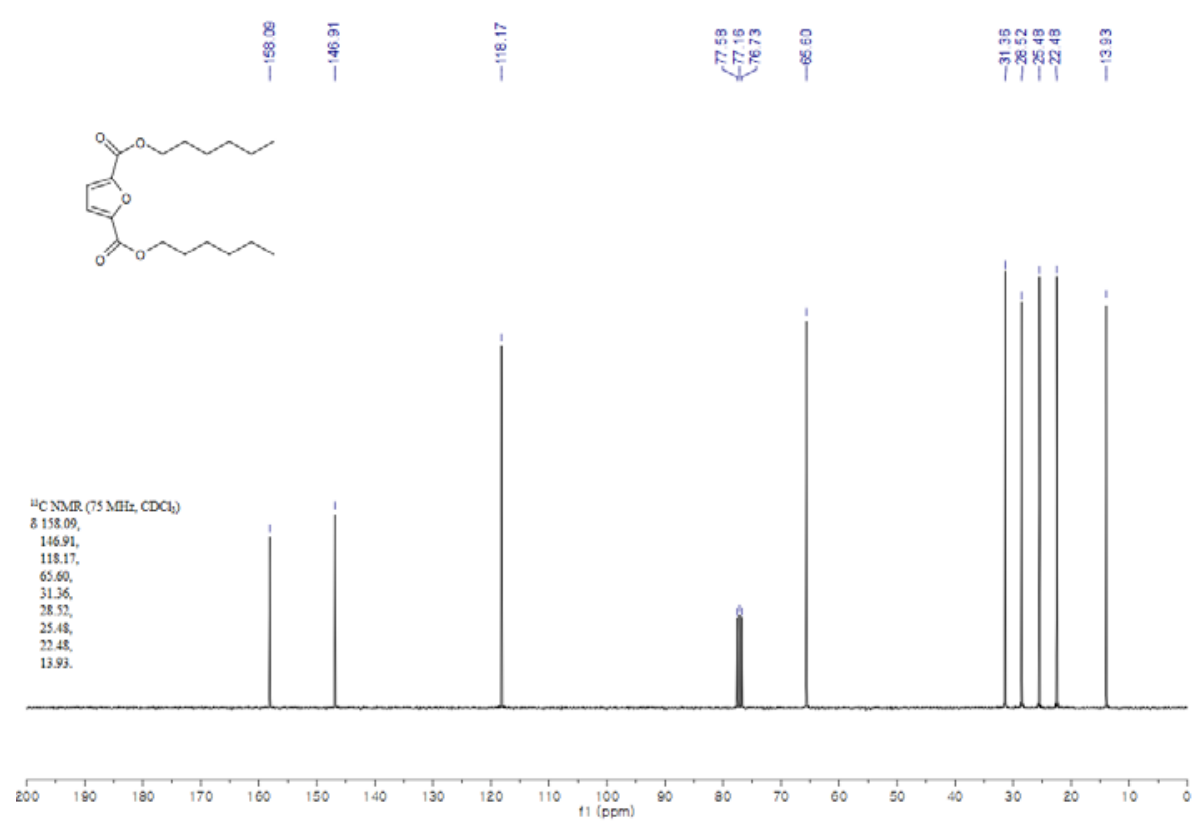

Figure S15. ${ }^{13} \mathrm{C}$-NMR spectrum of dihexyl furan-2,5-dicarboxylate 


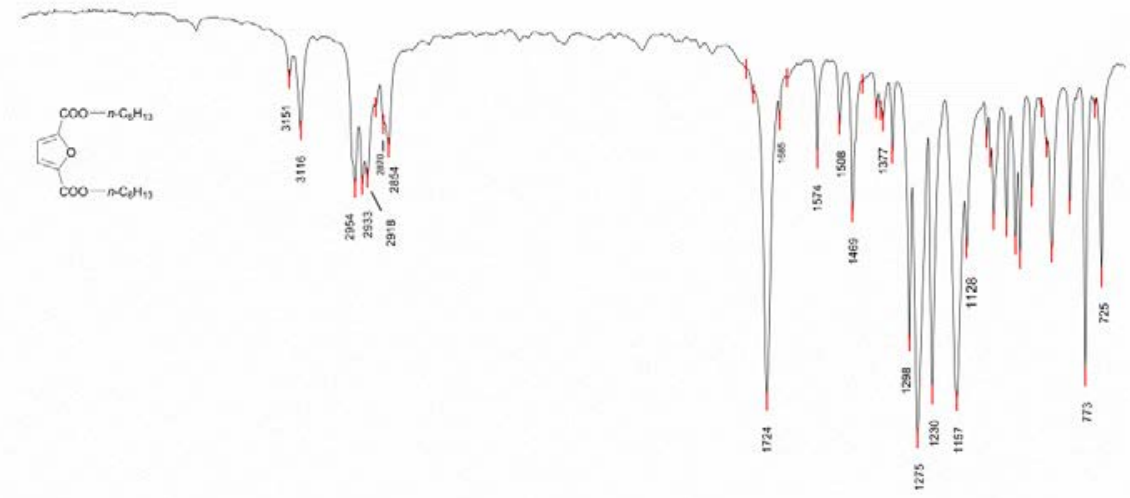

Wavenumber $\left(\mathrm{cm}^{-1}\right)$

Figure S16. Infrared spectrum of dihexyl furan-2,5-dicarboxylate

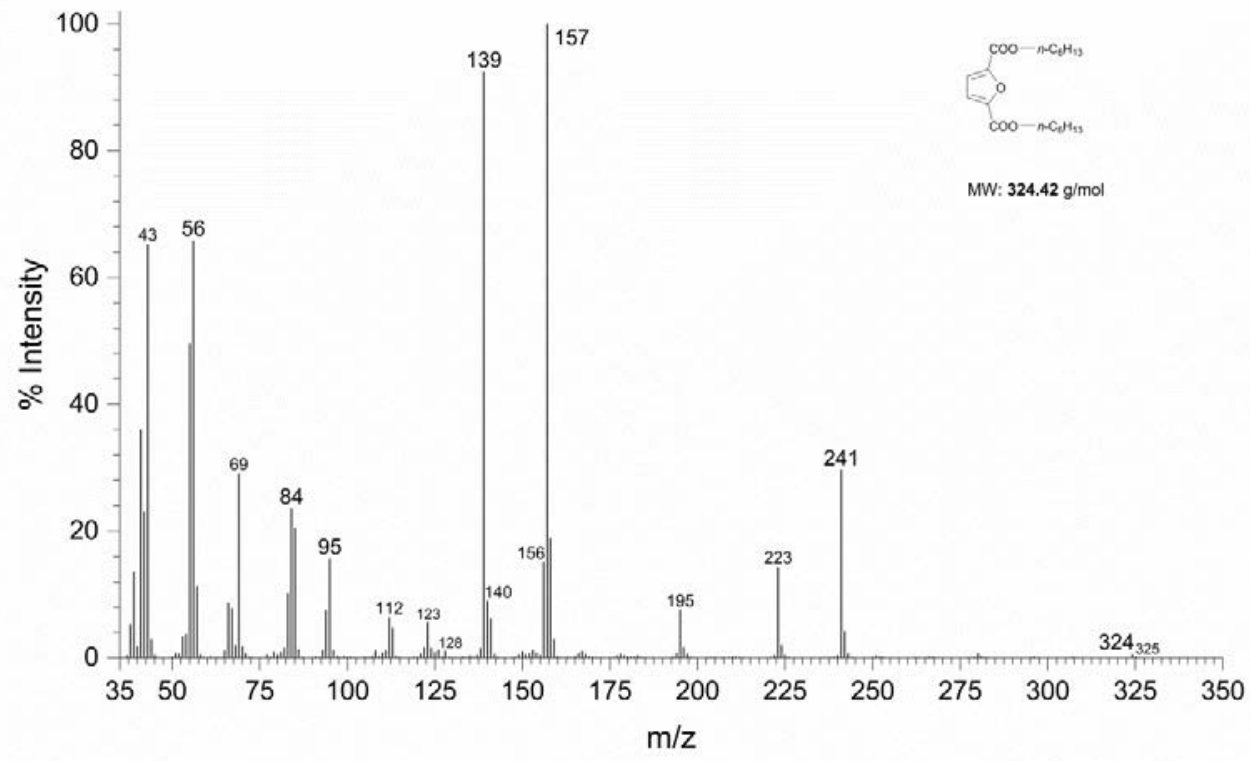

Figure S17. Mass spectrum of dihexyl furan-2,5-dicarboxylate 
D - DIHEPTYL FURAN-2,5-DICARBOXYLATE

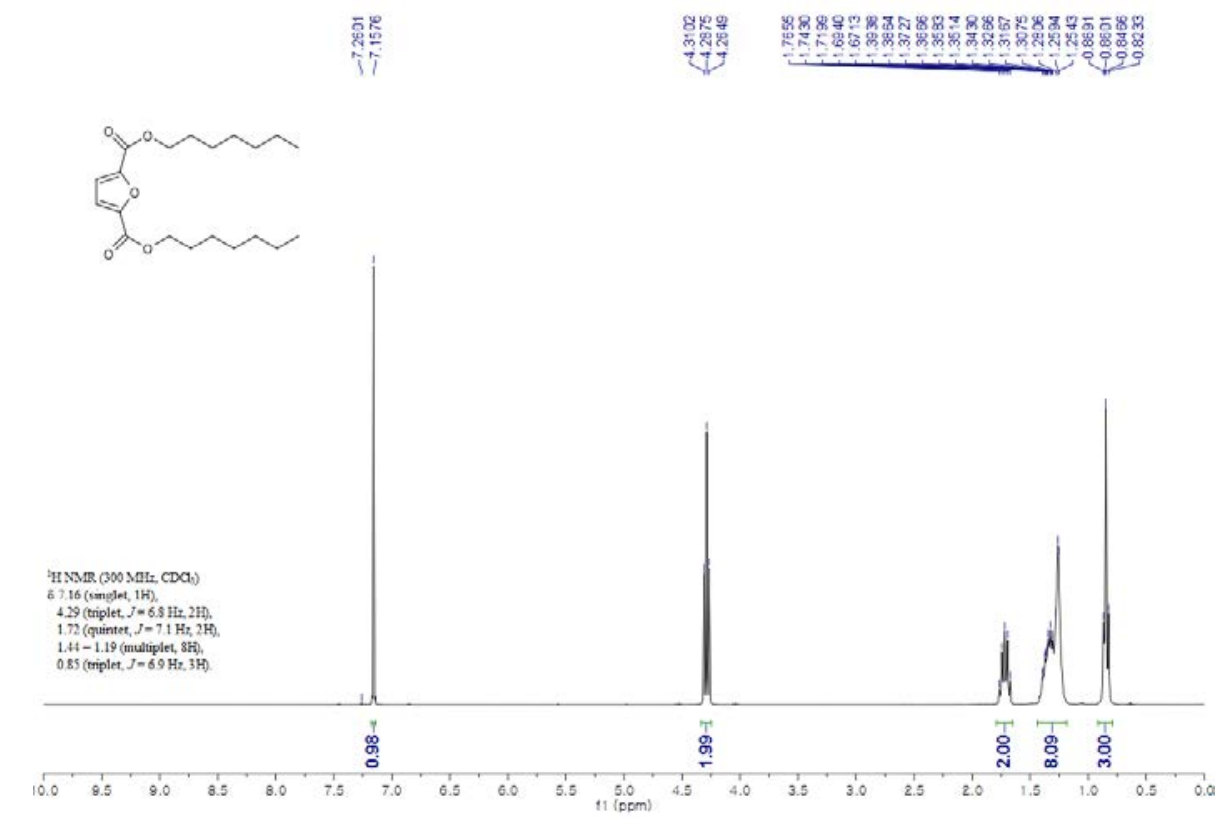

Figure S18. ${ }^{1} \mathrm{H}-\mathrm{NMR}$ spectrum of diheptyl furan-2,5-dicarboxylate

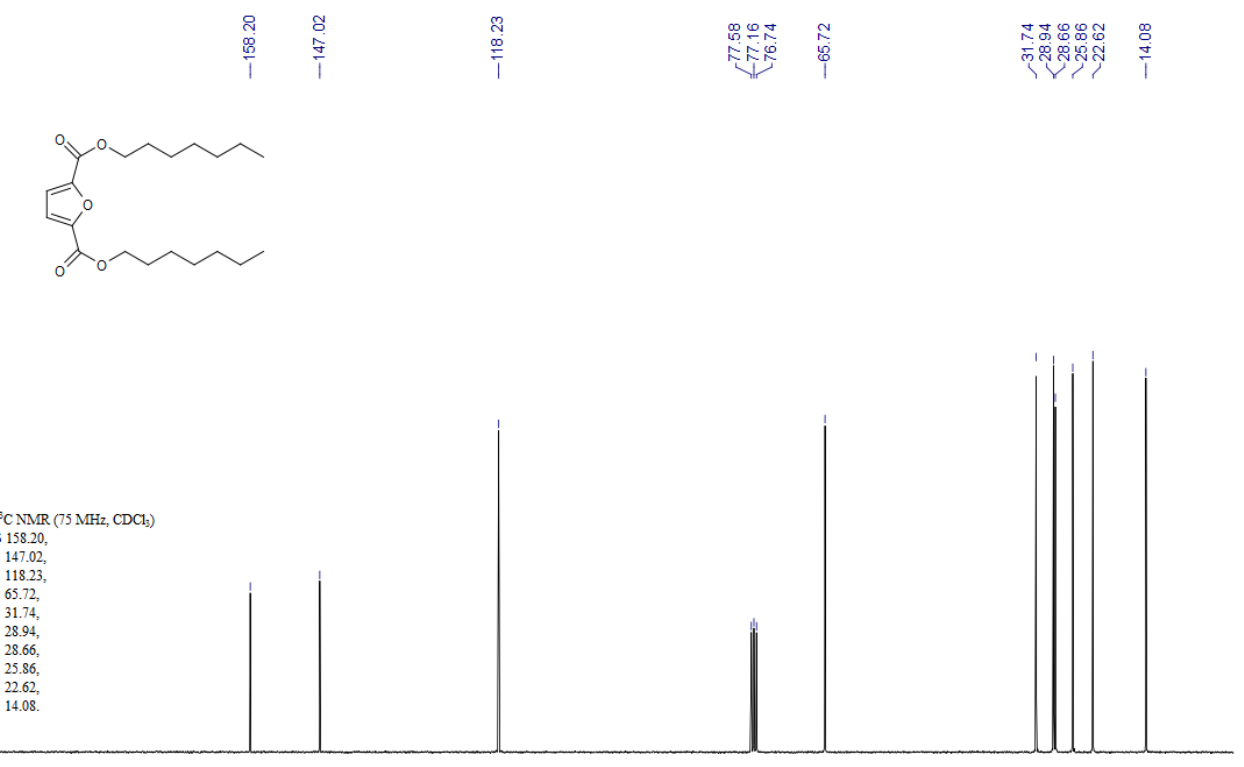

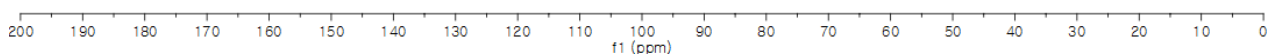

Figure S19. ${ }^{13} \mathrm{C}$-NMR spectrum of diheptyl furan-2,5-dicarboxylate 


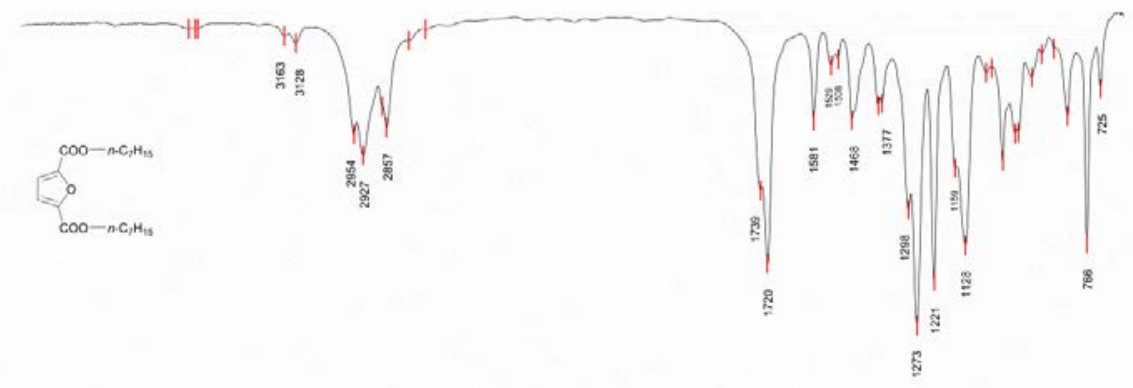

Figure S20. Infrared spectrum of diheptyl furan-2,5-dicarboxylate

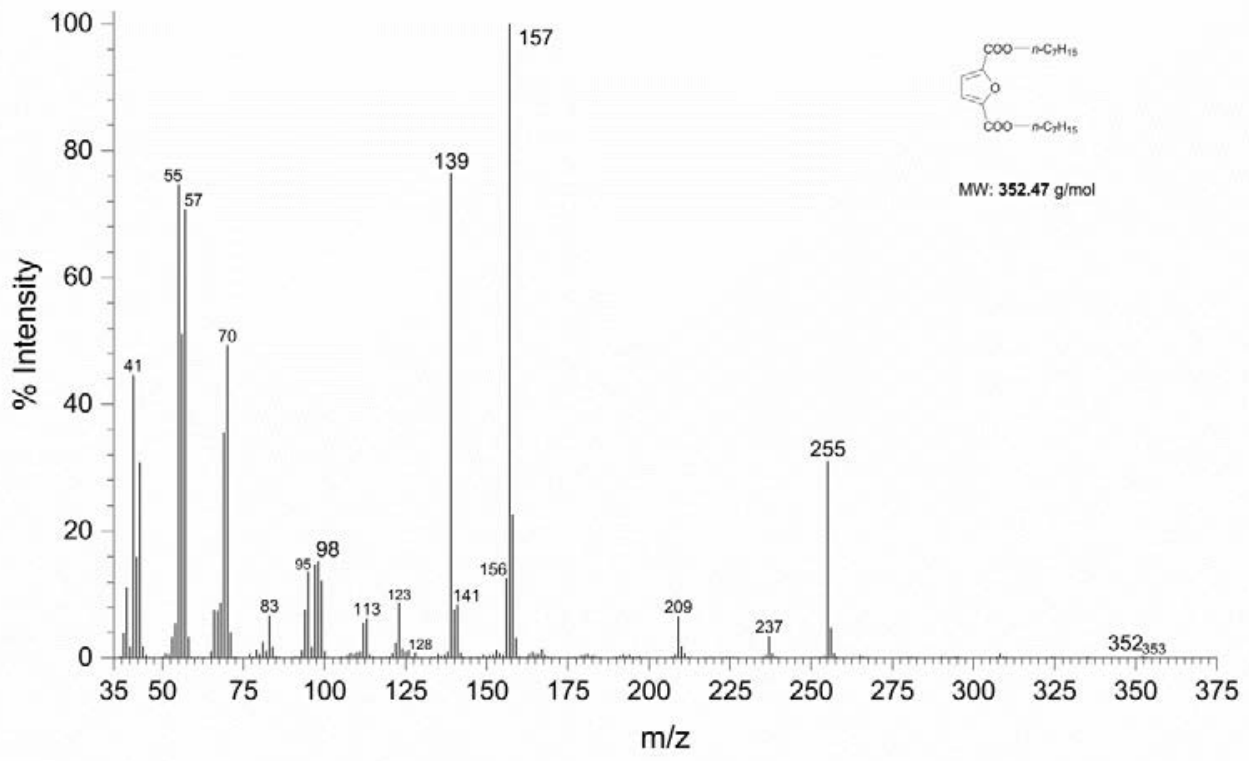

Figure S21. Mass spectrum of diheptyl furan-2,5-dicarboxylate 


\section{E - DIOCTYL FURAN-2,5-DICARBOXYLATE}

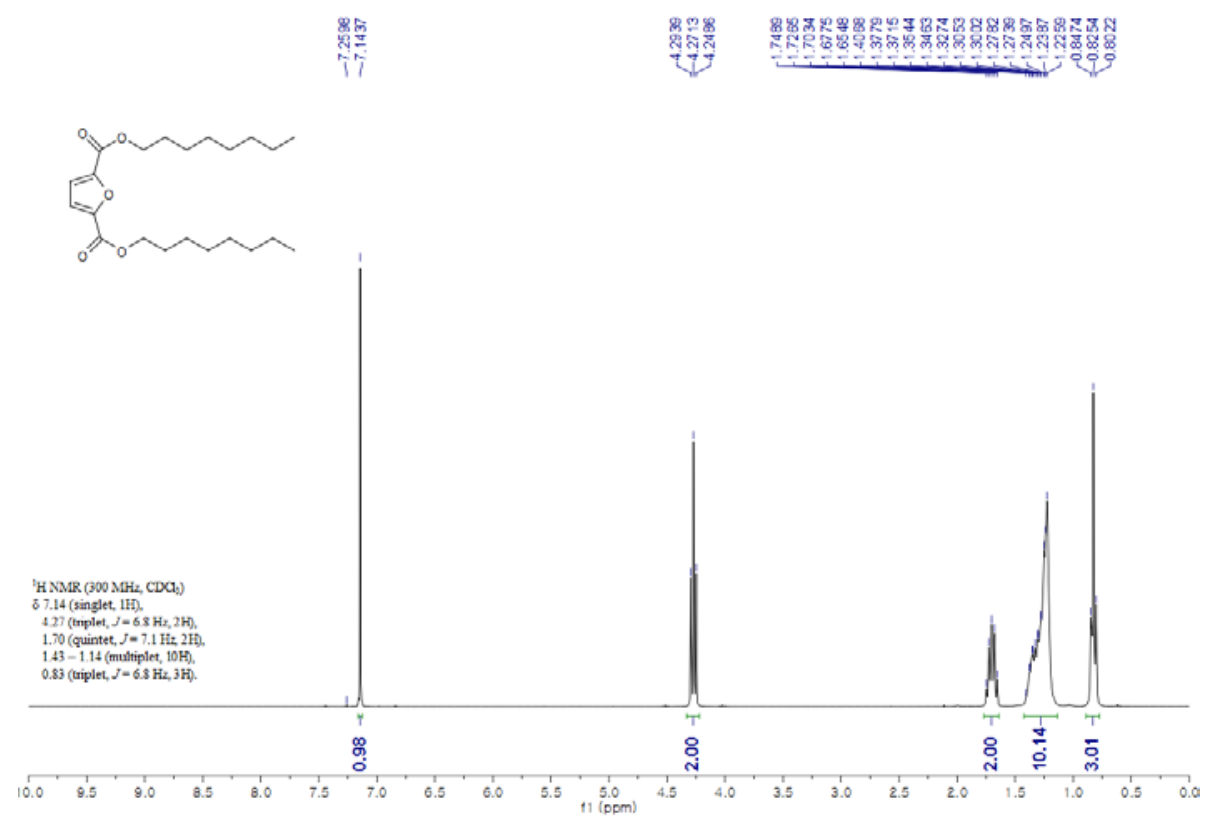

Figure S22. ${ }^{1} \mathrm{H}-\mathrm{NMR}$ spectrum of dioctyl furan-2,5-dicarboxylate

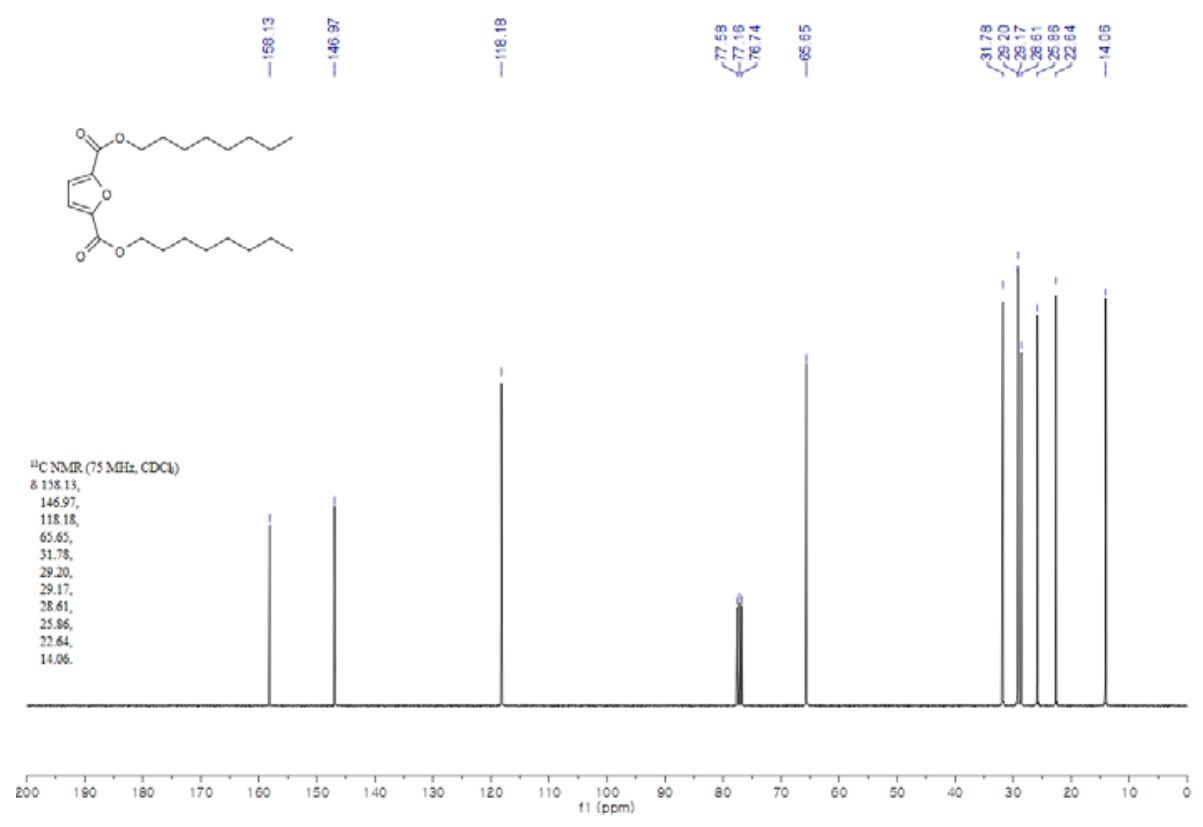

Figure S23. ${ }^{13} \mathrm{C}$-NMR spectrum of dioctyl furan-2,5-dicarboxylate 


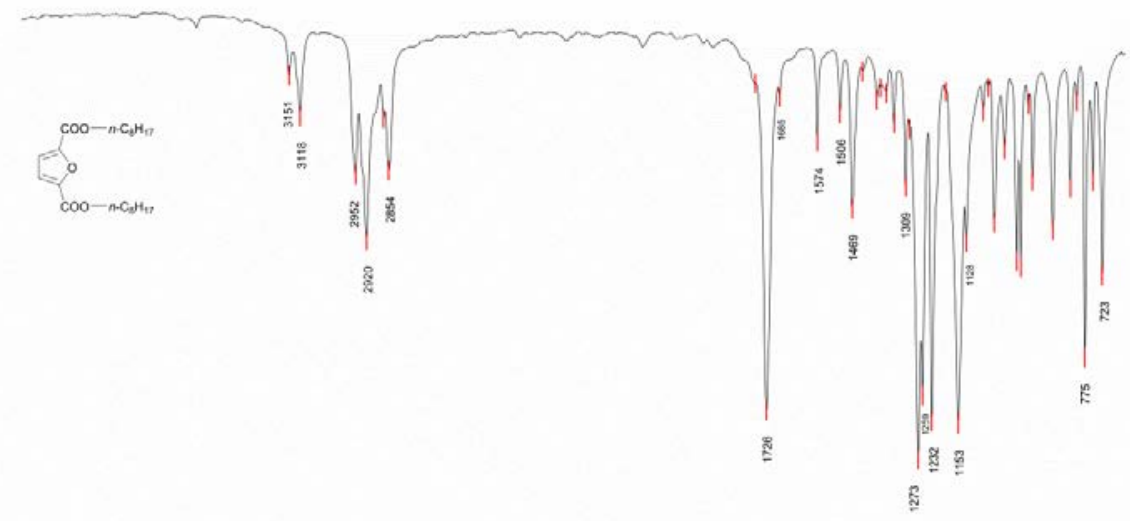

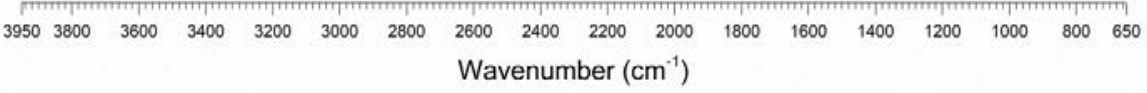

Figure S24. Infrared spectrum of dioctyl furan-2,5-dicarboxylate

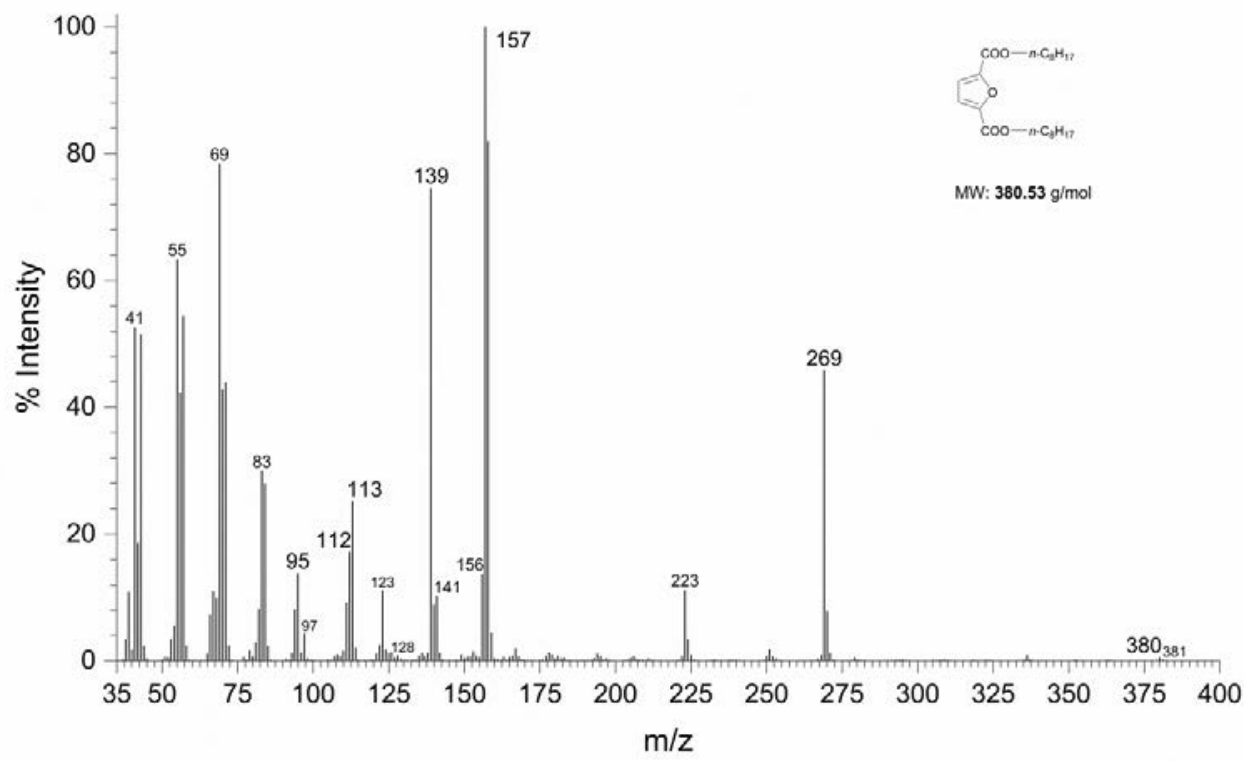

Figure S25. Mass spectrum of dioctyl furan-2,5-dicarboxylate 


\section{F - DIISOAMYL FURAN-2,5-DICARBOXYLATE (2,5-DIAF)}
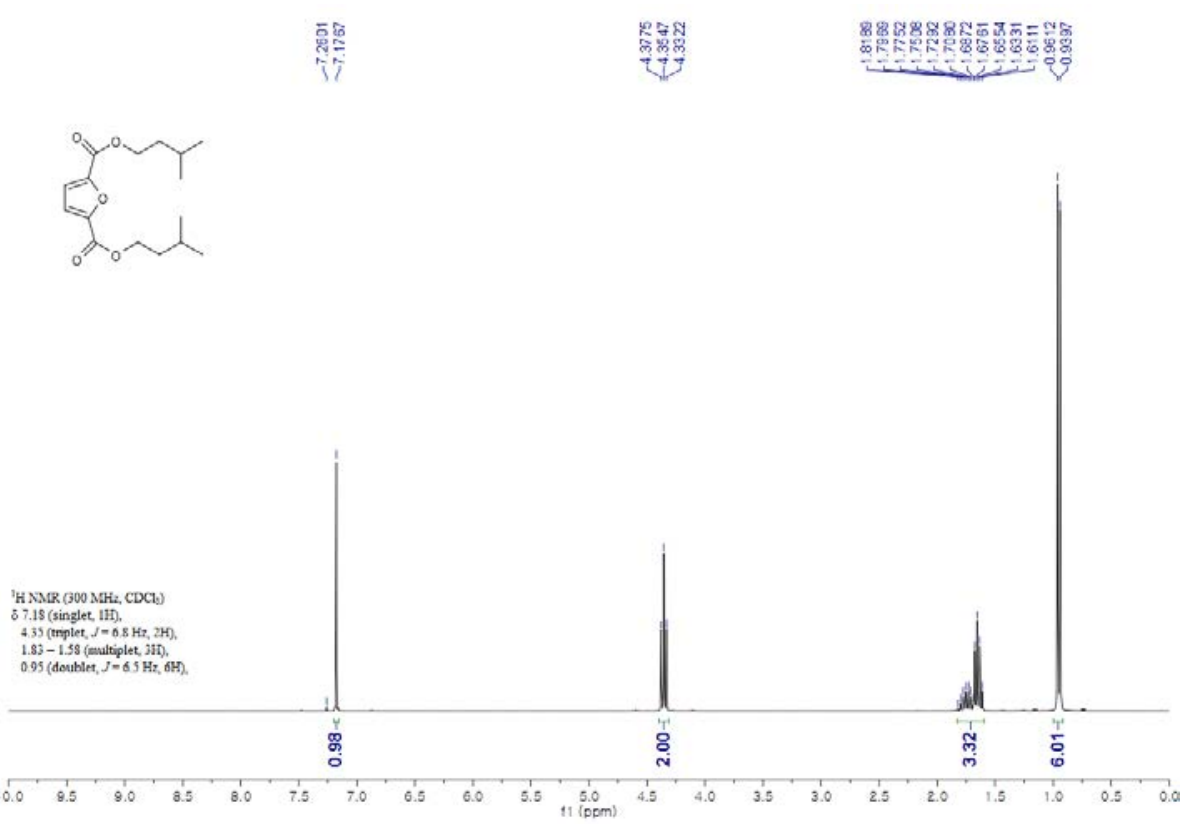

Figure S26. ${ }^{1}$ H-NMR spectrum of diisoamyl furan-2,5-dicarboxylate (2,5-DIAF)

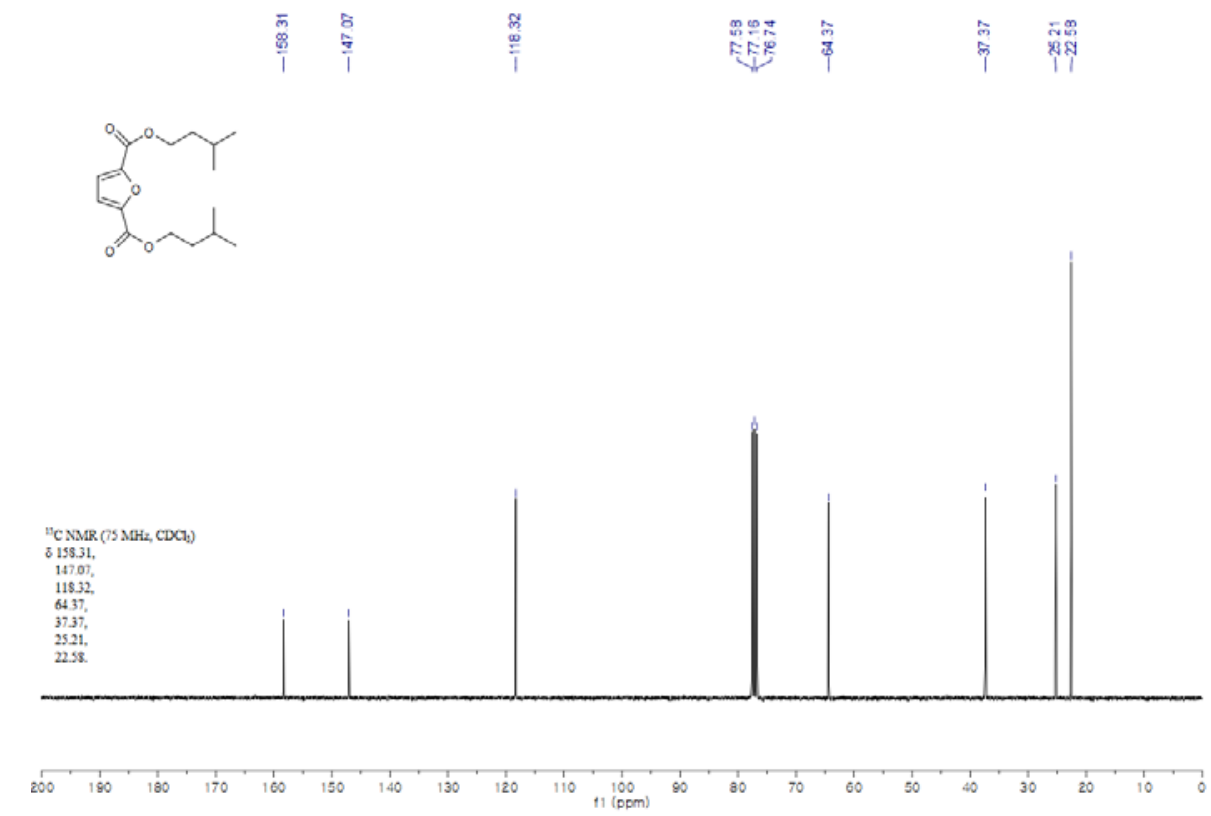

Figure S27. ${ }^{13} \mathrm{C}-\mathrm{NMR}$ spectrum of diisoamyl furan-2,5-dicarboxylate (2,5-DIAF) 

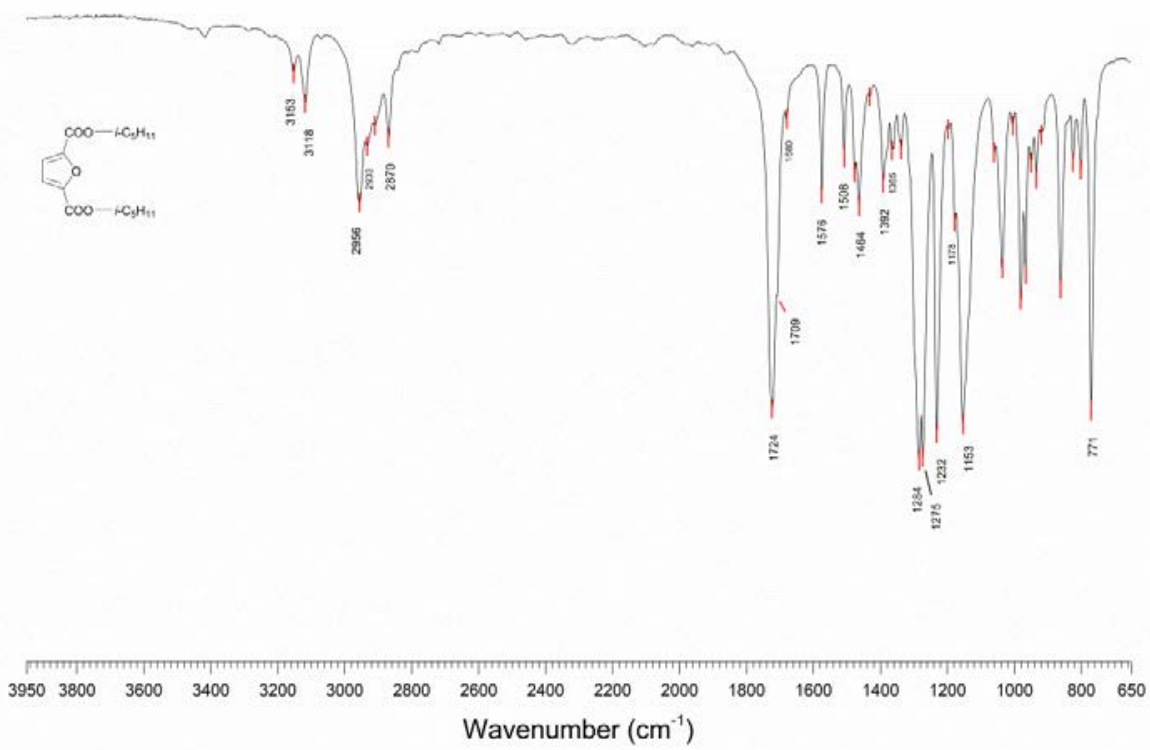

Figure S28. Infrared spectrum of diisoamyl furan-2,5-dicarboxylate (2,5-DIAF)

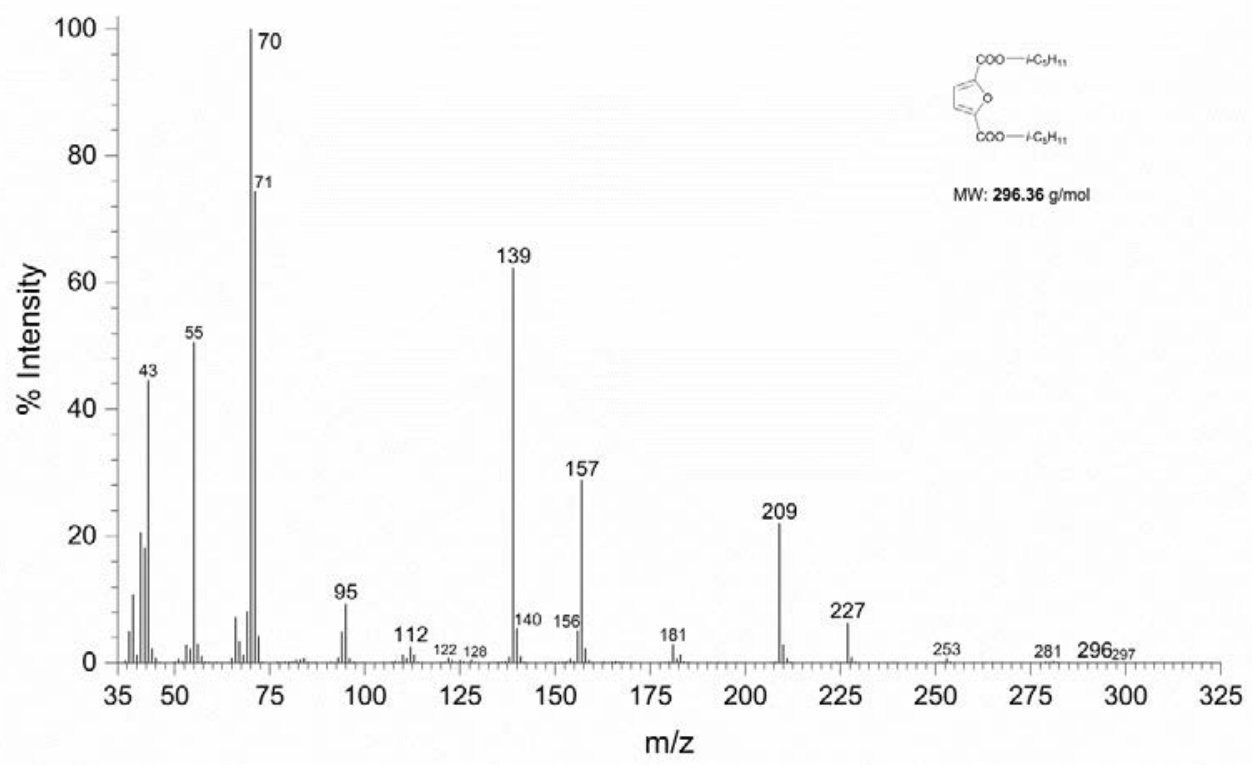

Figure S29. Mass spectrum of diisoamyl furan-2,5-dicarboxylate (2,5-DIAF) 


\section{G - DI(2-ETHYLHEXYL) FURAN-2,5-DICARBOXYLATE}

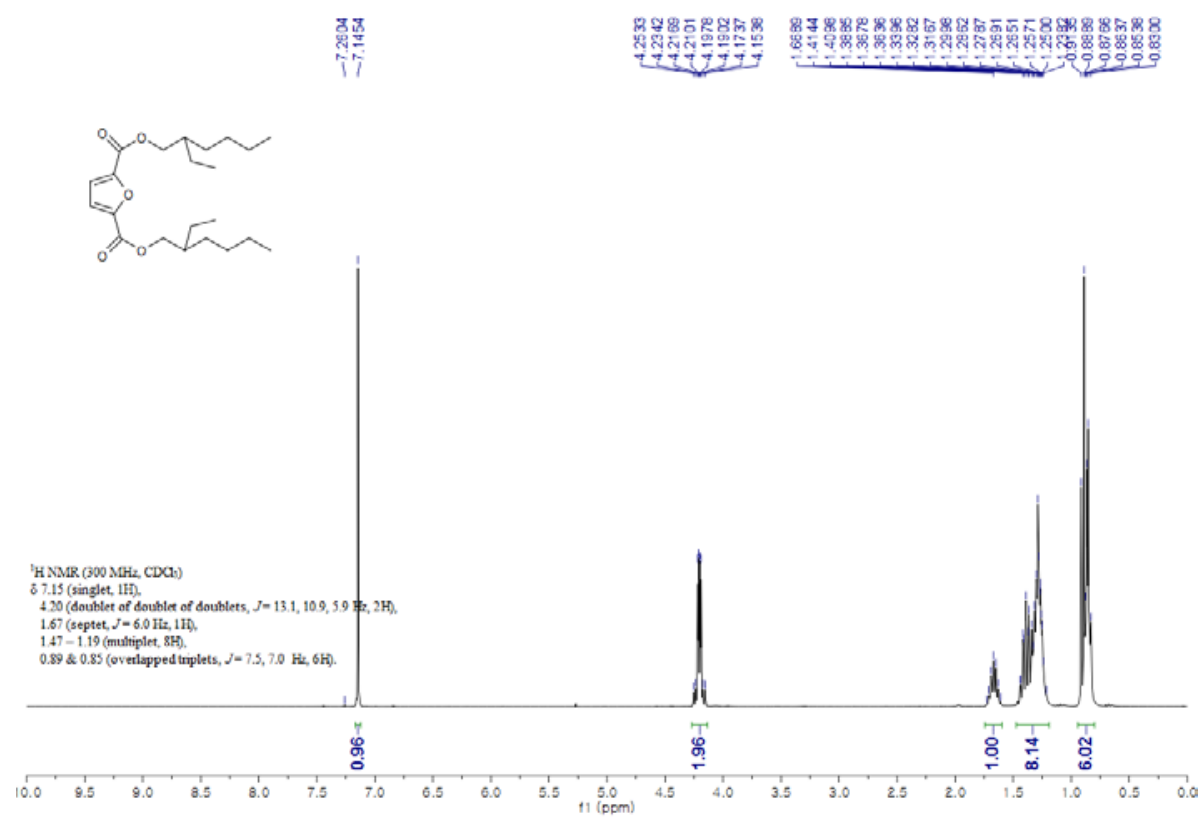

Figure S30. ${ }^{1} \mathrm{H}-\mathrm{NMR}$ spectrum of di(2-ethylhexyl) furan-2,5-dicarboxylate

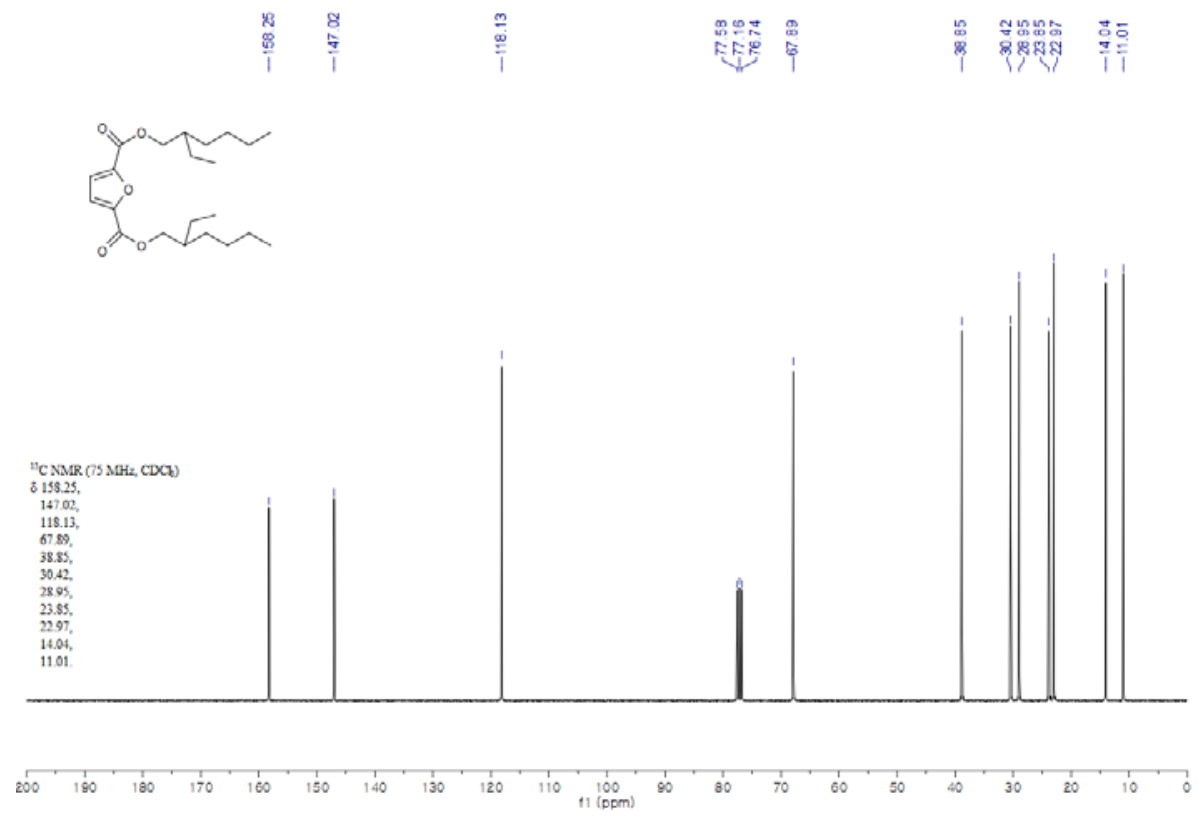

Figure S31. ${ }^{13} \mathrm{C}-\mathrm{NMR}$ spectrum of di(2-ethylhexyl) furan-2,5-dicarboxylate 

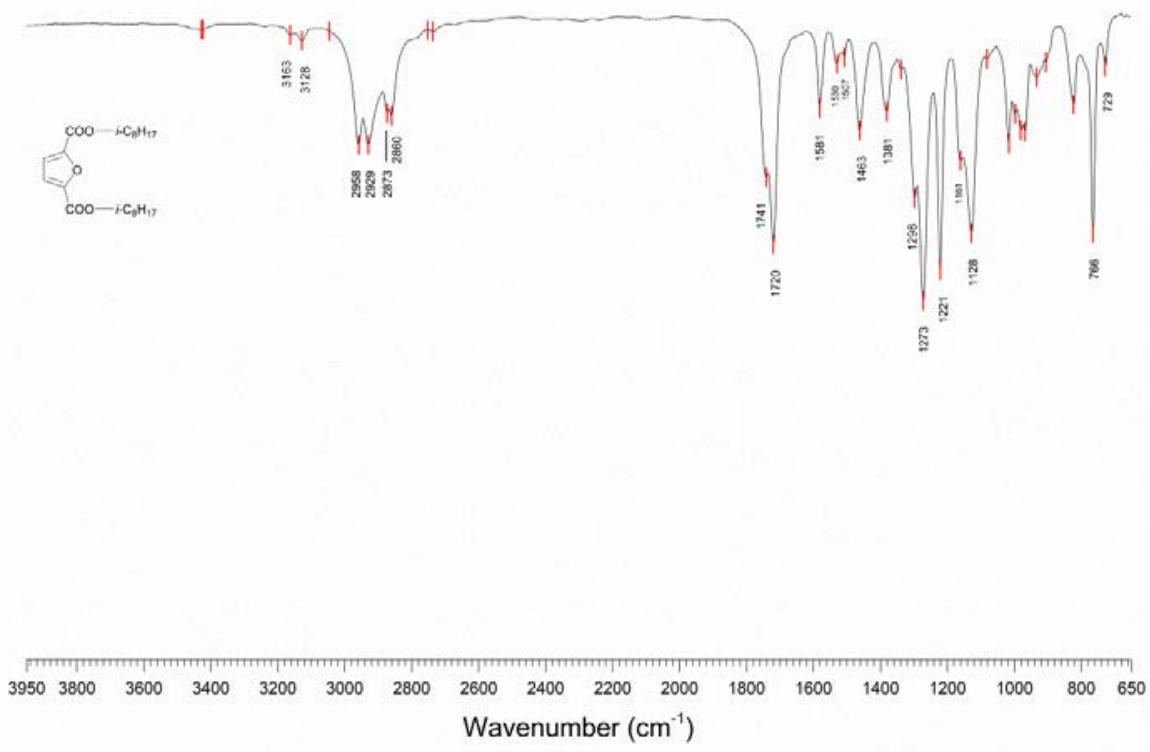

Figure S32. Infrared spectrum of di(2-ethylhexyl) furan-2,5-dicarboxylate

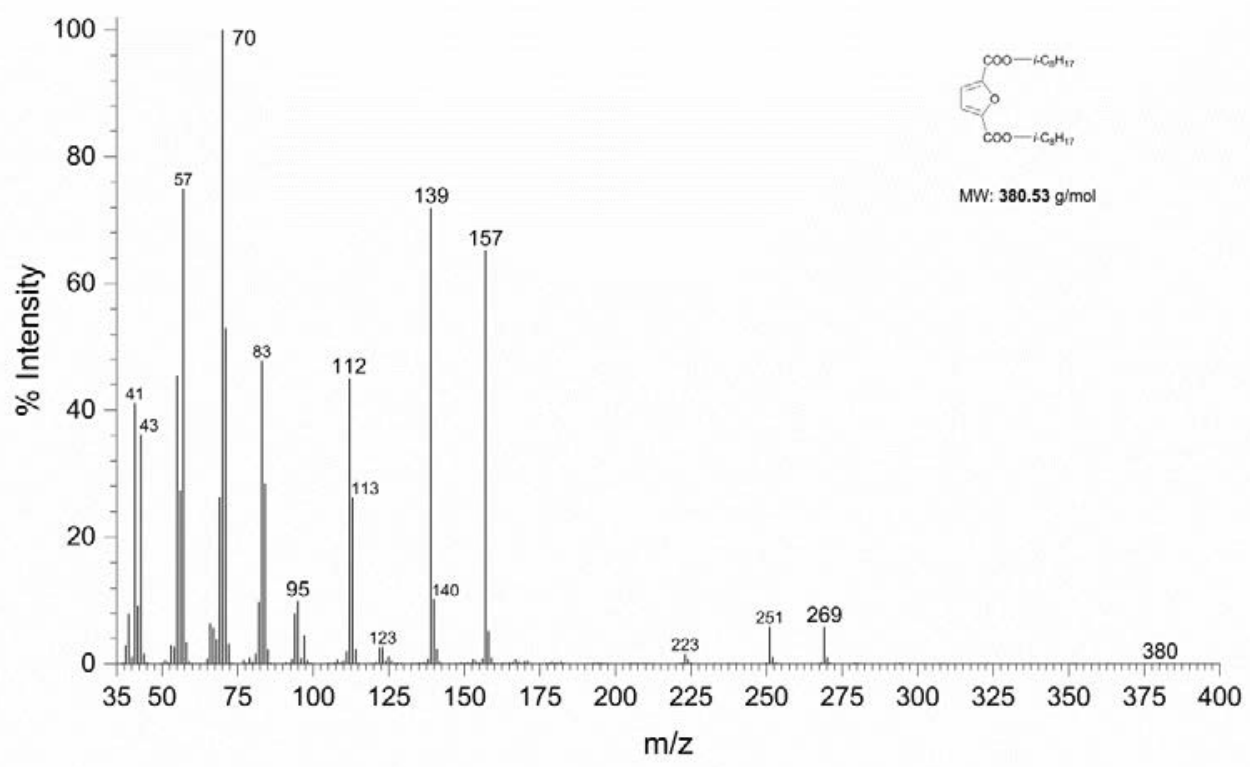

Figure S33. Mass spectrum of di(2-ethylhexyl) furan-2,5-dicarboxylate 


\section{Dialkyl furan-2,3-dicarboxylates (2,3-DAFs)}

A - DIBUTYL FURAN-2,3-DICARBOXYLATE (2,3-DBF)
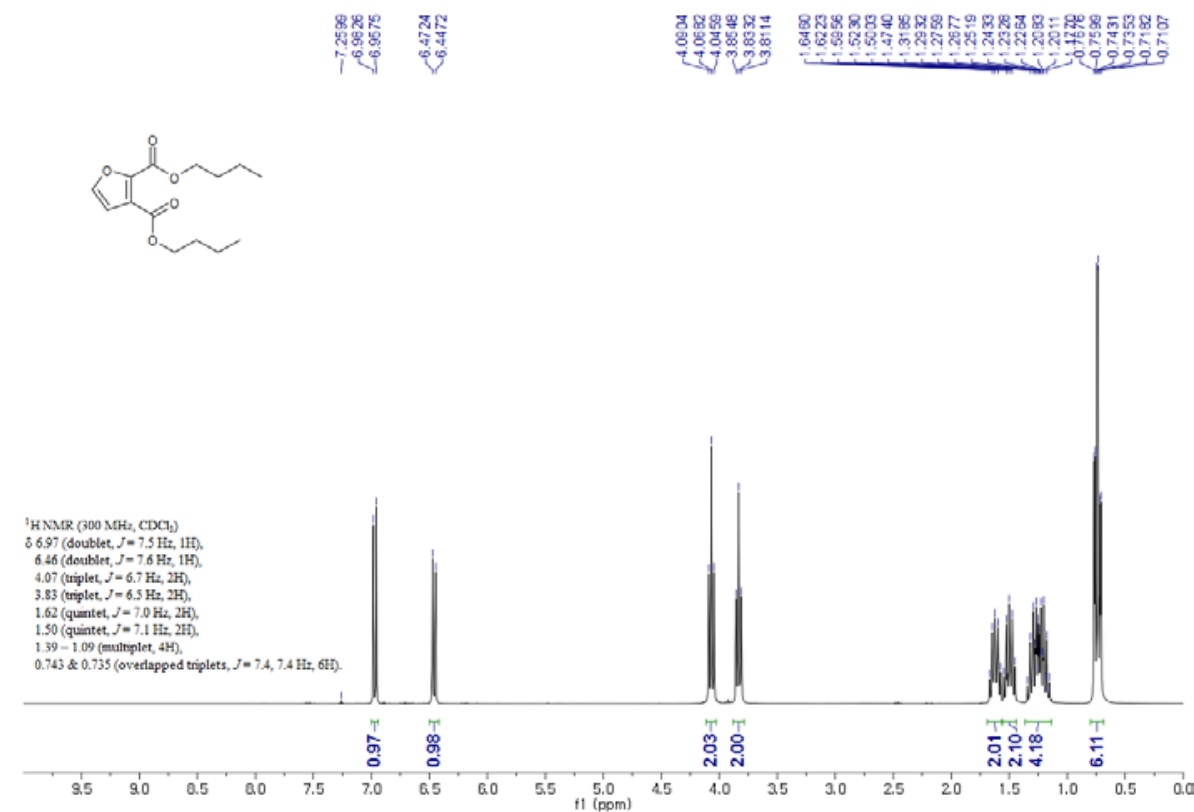

Figure S34. ${ }^{1} \mathrm{H}-\mathrm{NMR}$ spectrum of dibutyl furan-2,3-dicarboxylate (2,3-DBF)

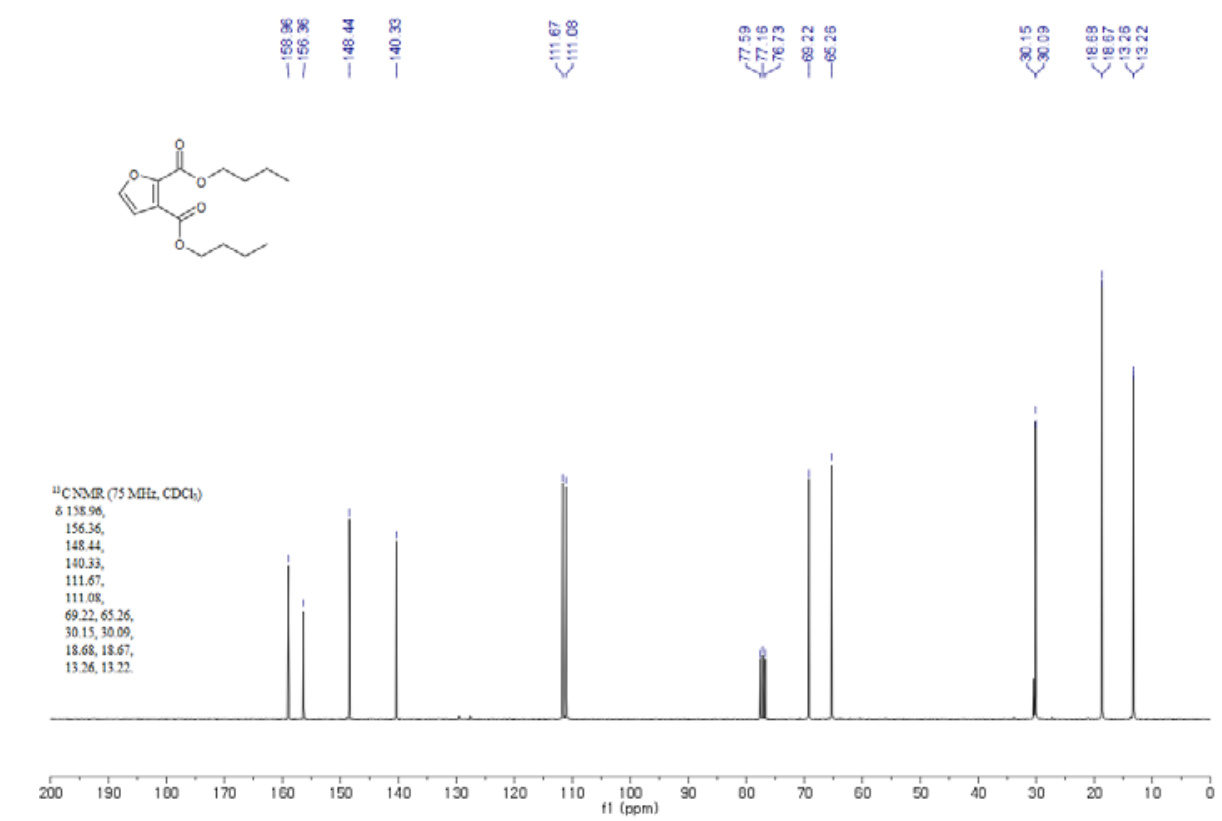

Figure S35. ${ }^{13} \mathrm{C}-\mathrm{NMR}$ spectrum of dibutyl furan-2,3-dicarboxylate (2,3-DBF) 

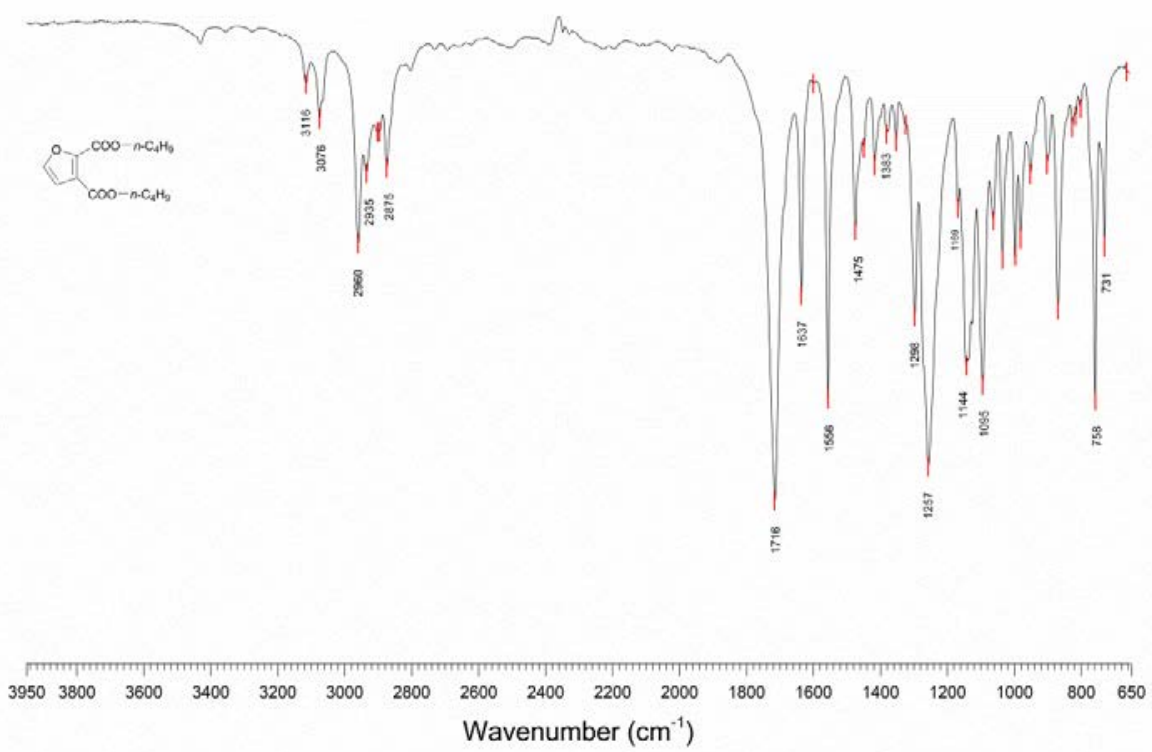

Figure S36. Infrared spectrum of dibutyl furan-2,3-dicarboxylate (2,3-DBF)

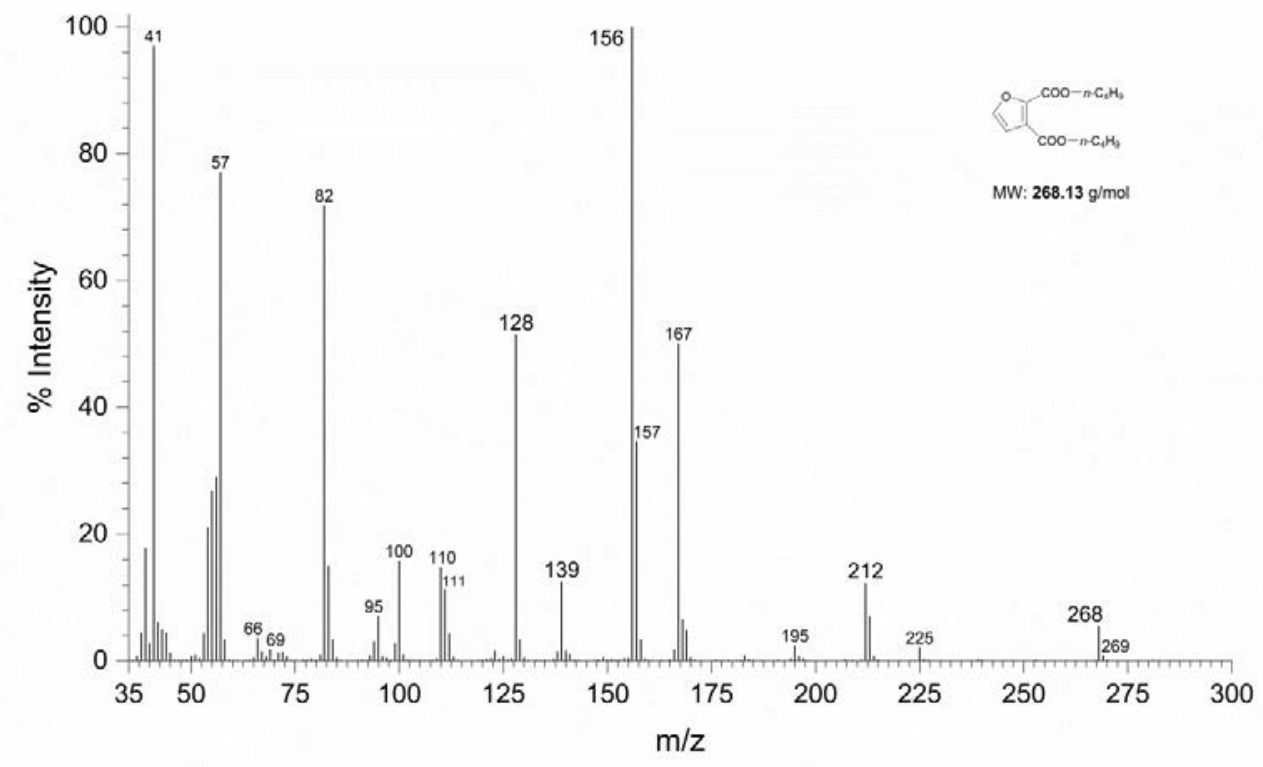

Figure S37. Mass spectrum of dibutyl furan-2,3-dicarboxylate (2,3-DBF) 
B - DIPENTYL FURAN-2,3-DICARBOXYLATE
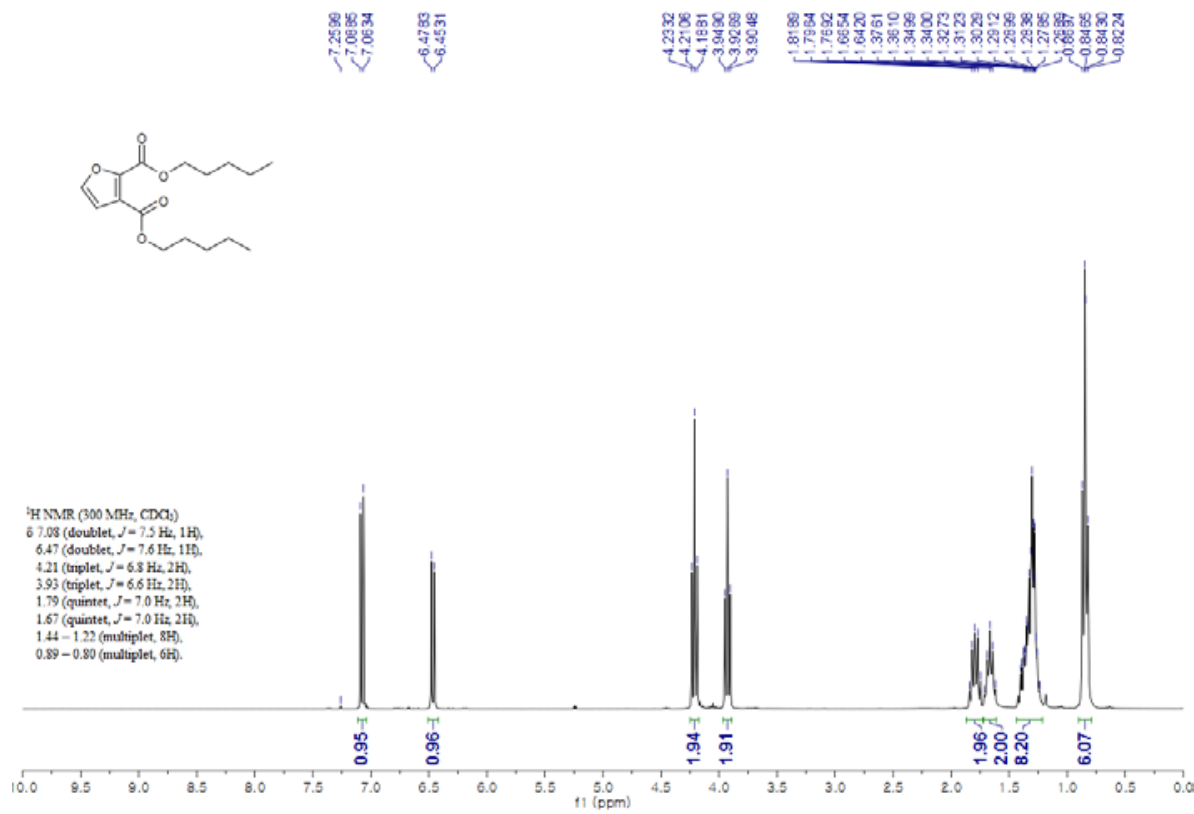

Figure S38. ${ }^{1} \mathrm{H}-\mathrm{NMR}$ spectrum of dipentyl furan-2,3-dicarboxylate

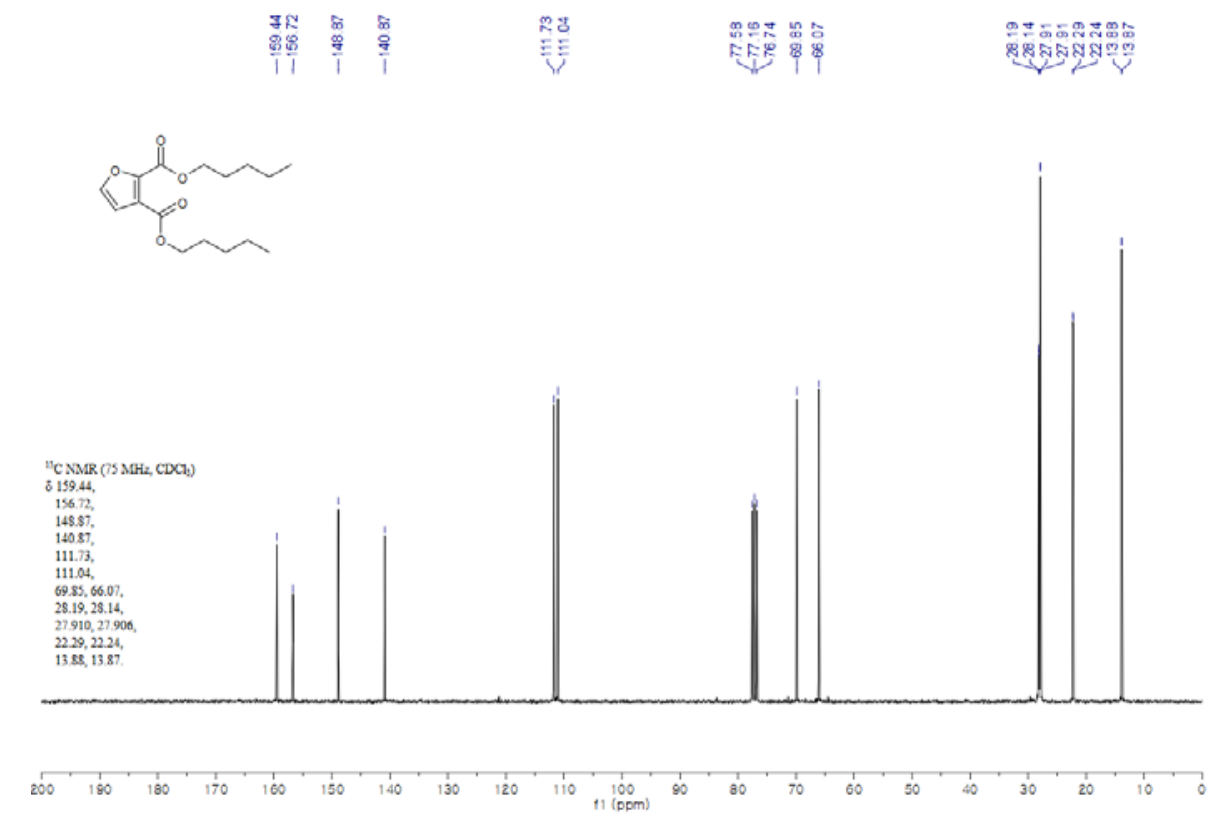

Figure S39. ${ }^{13}$ C-NMR spectrum of dipentyl furan-2,3-dicarboxylate 


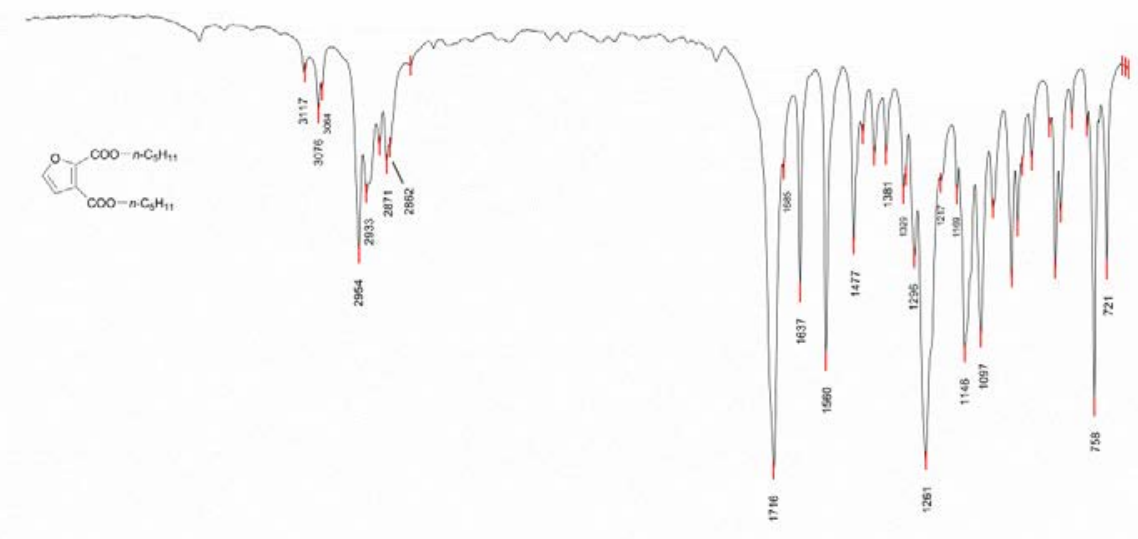

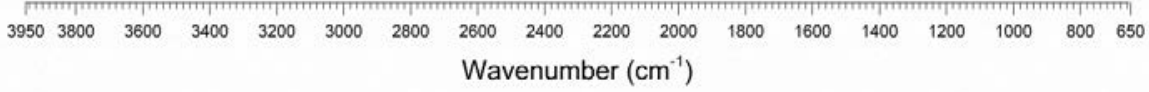

Figure S40. Infrared spectrum of dipentyl furan-2,3-dicarboxylate

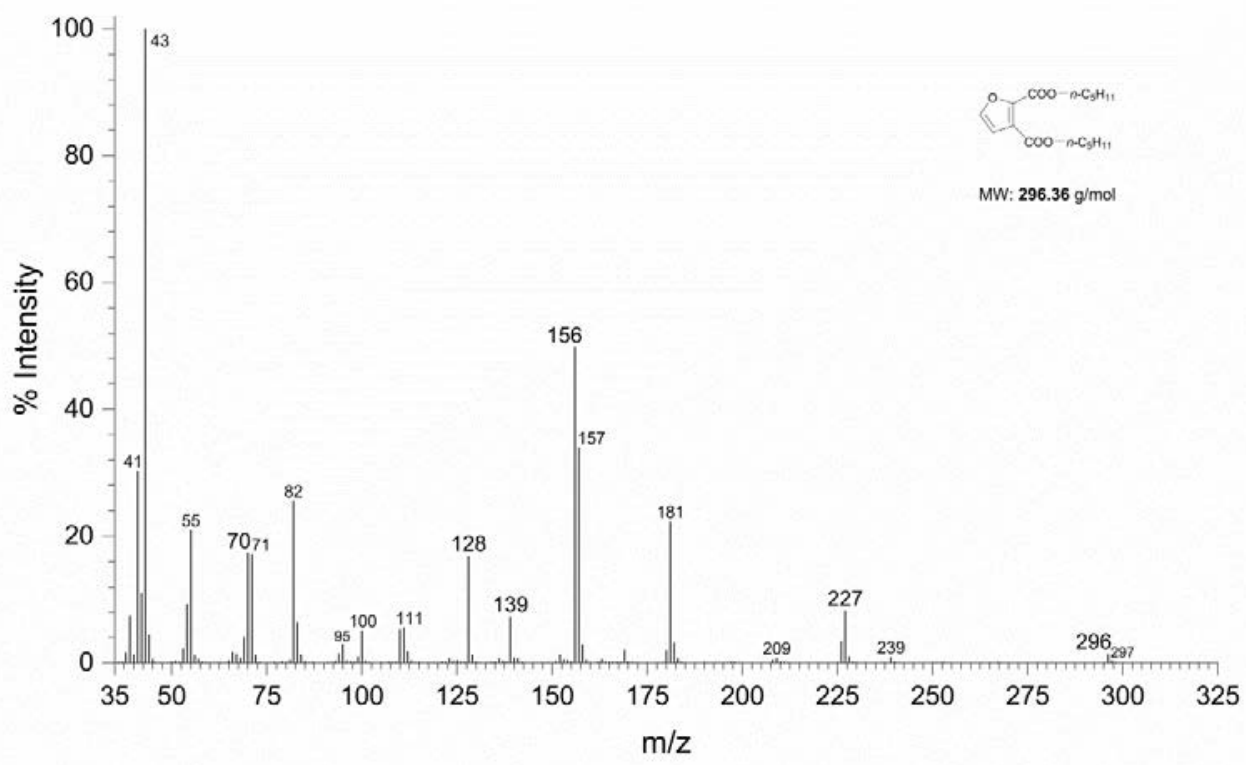

Figure S41. Mass spectrum of dipentyl furan-2,3-dicarboxylate 


\section{C - DIHEXYL FURAN-2,3-DICARBOXYLATE}
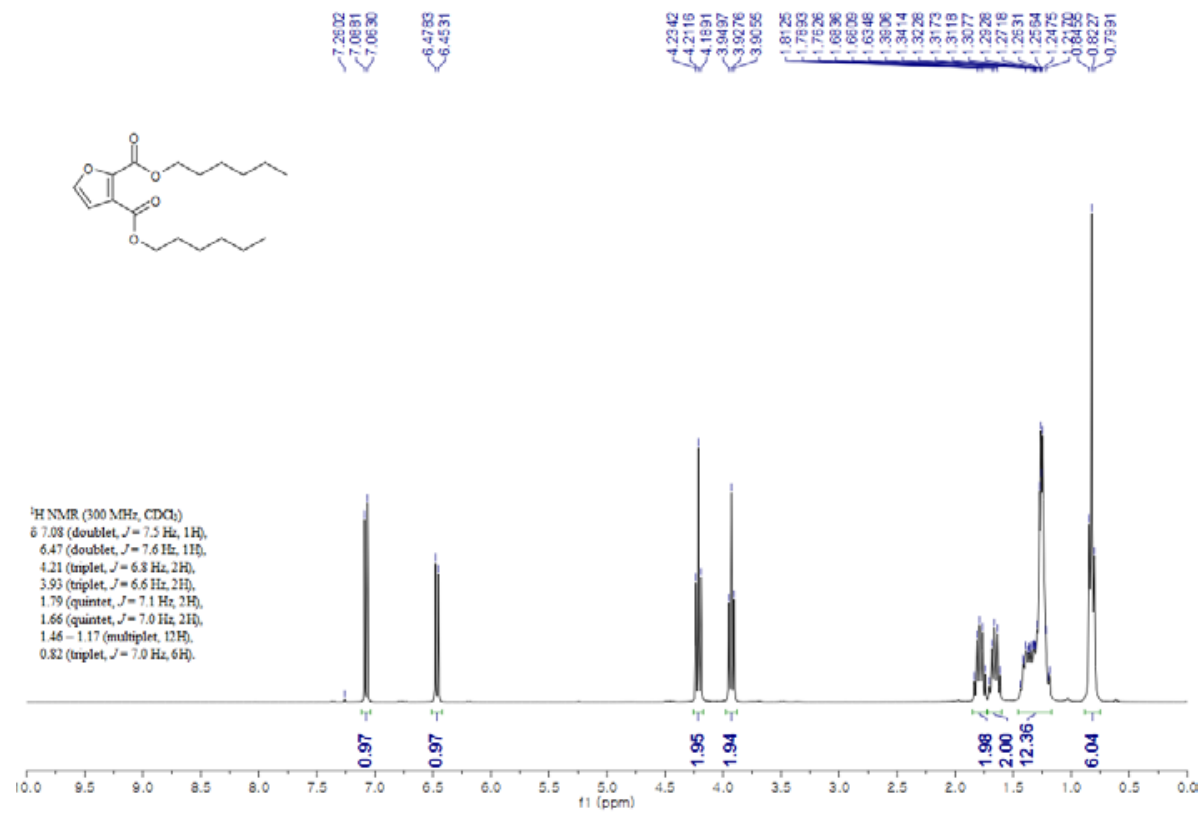

Figure S42. ${ }^{1} \mathrm{H}-\mathrm{NMR}$ spectrum of dihexyl furan-2,3-dicarboxylate

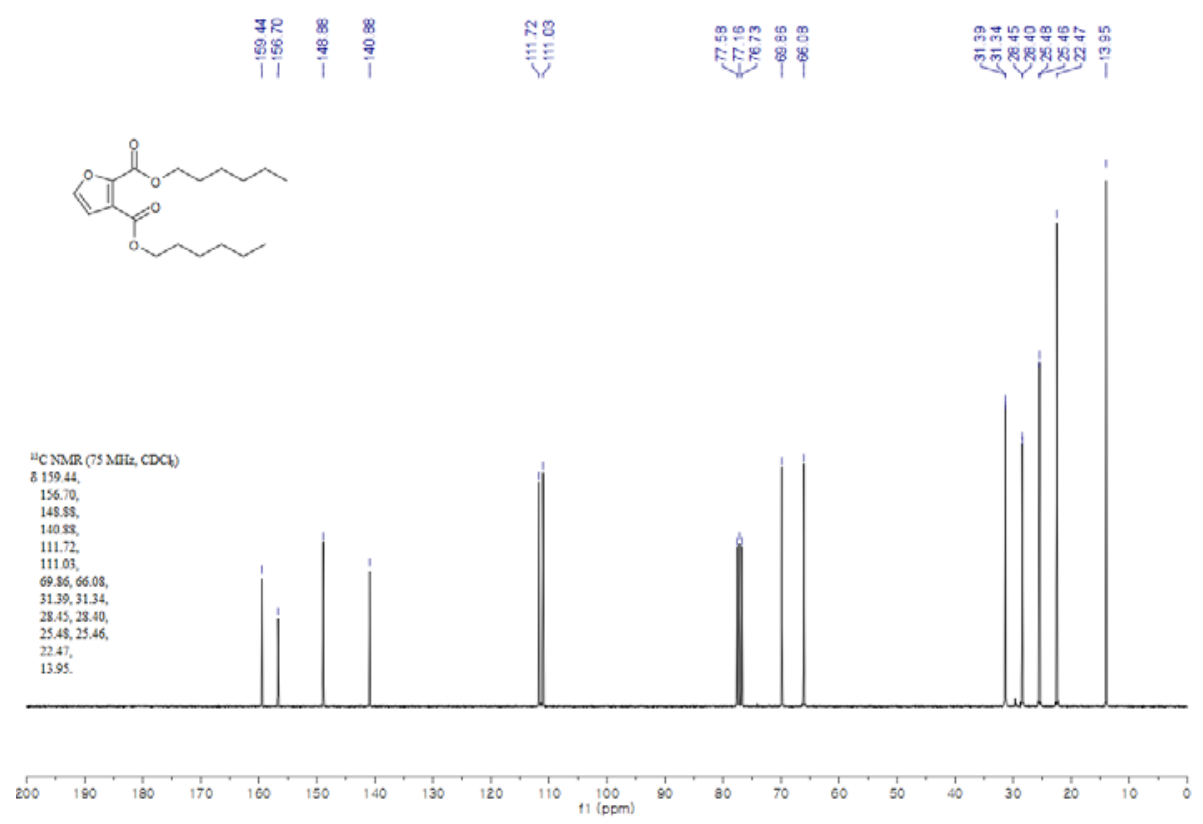

Figure S43. ${ }^{13} \mathrm{C}$-NMR spectrum of dihexyl furan-2,3-dicarboxylate 

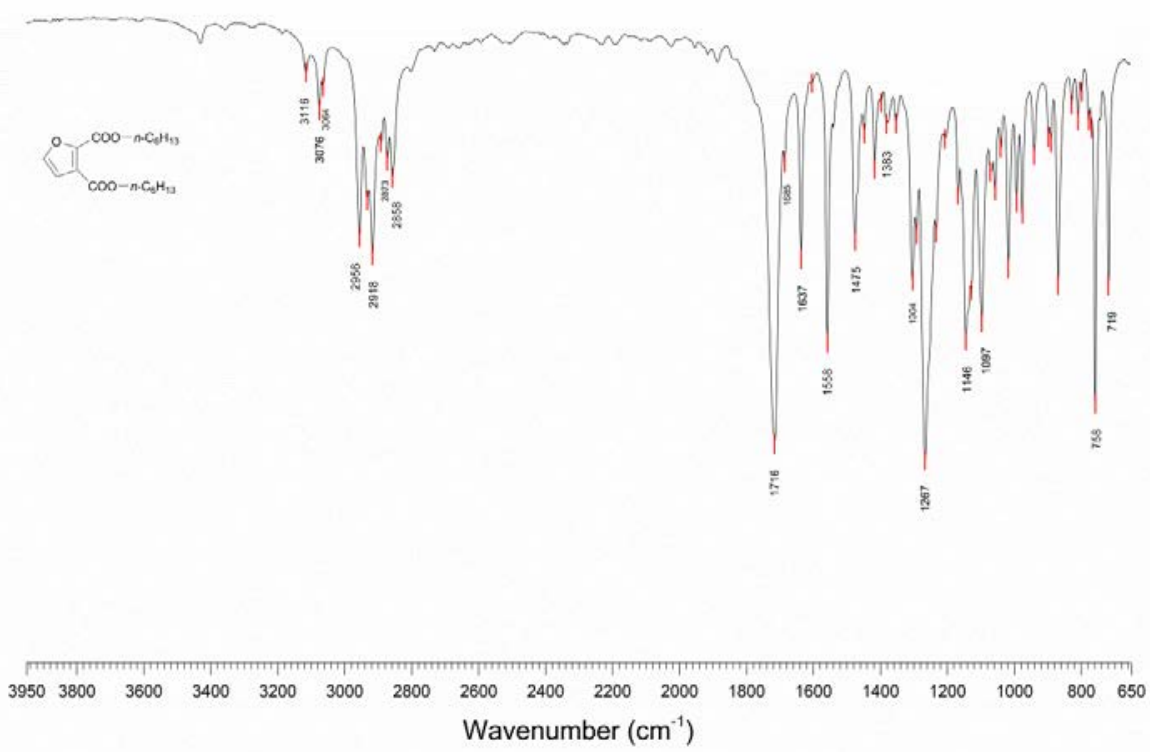

Figure S44. Infrared spectrum of dihexyl furan-2,3-dicarboxylate

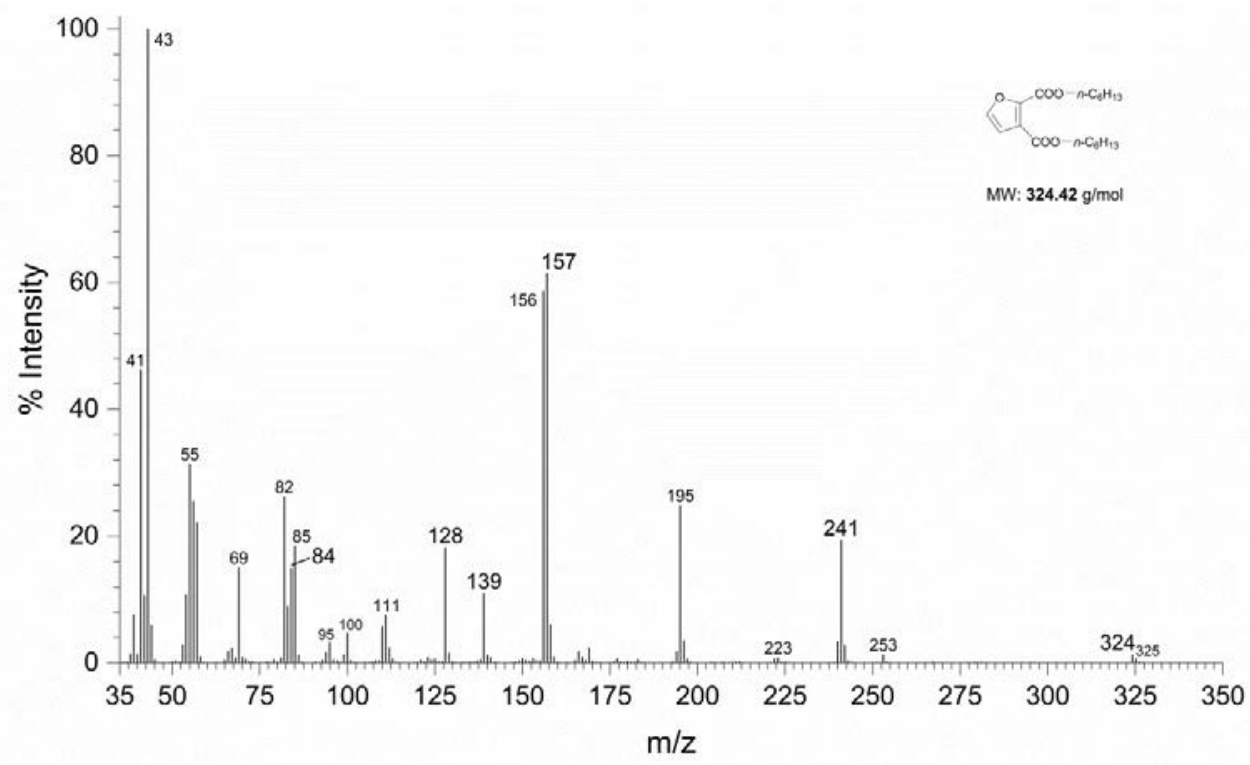

Figure S45. Mass spectrum of dihexyl furan-2,3-dicarboxylate 
D - DIHEPTYL FURAN-2,3-DICARBOXYLATE

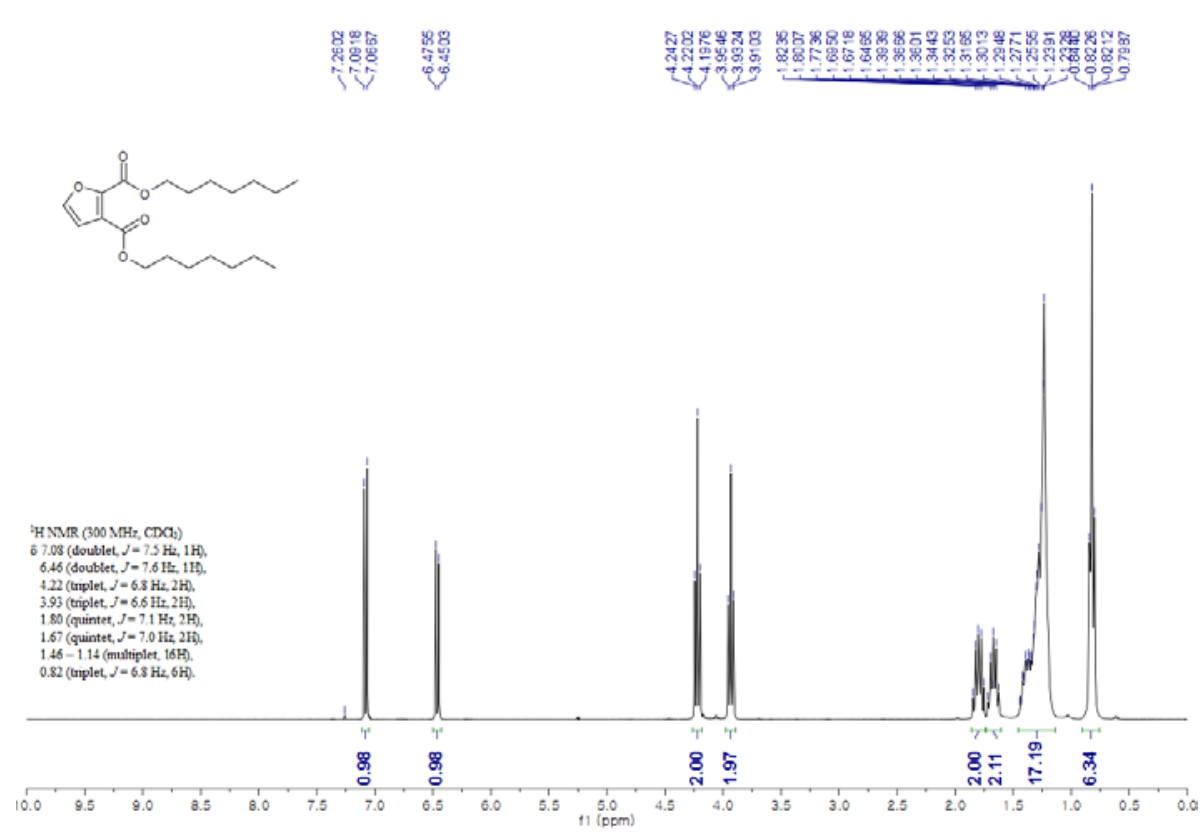

Figure S46. ${ }^{1} \mathrm{H}$-NMR spectrum of diheptyl furan-2,3-dicarboxylate

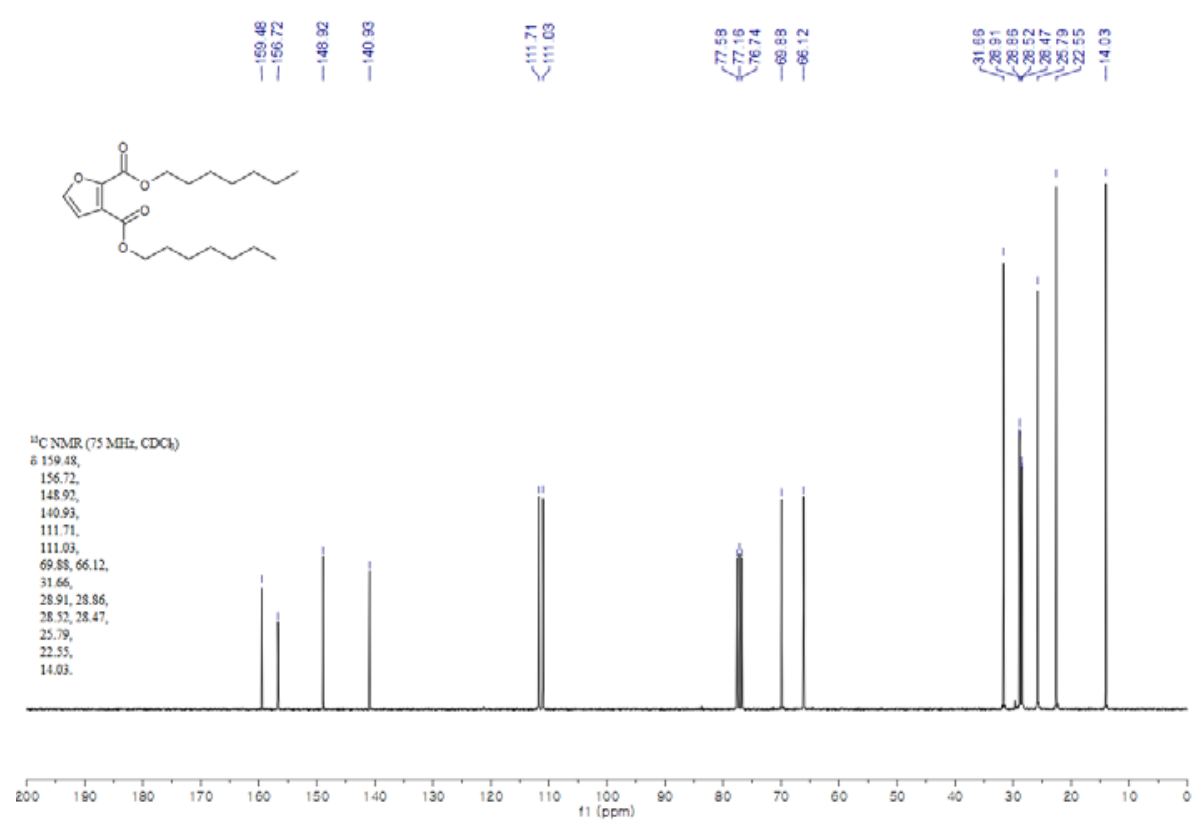

Figure S47. ${ }^{13} \mathrm{C}$-NMR spectrum of diheptyl furan-2,3-dicarboxylate 


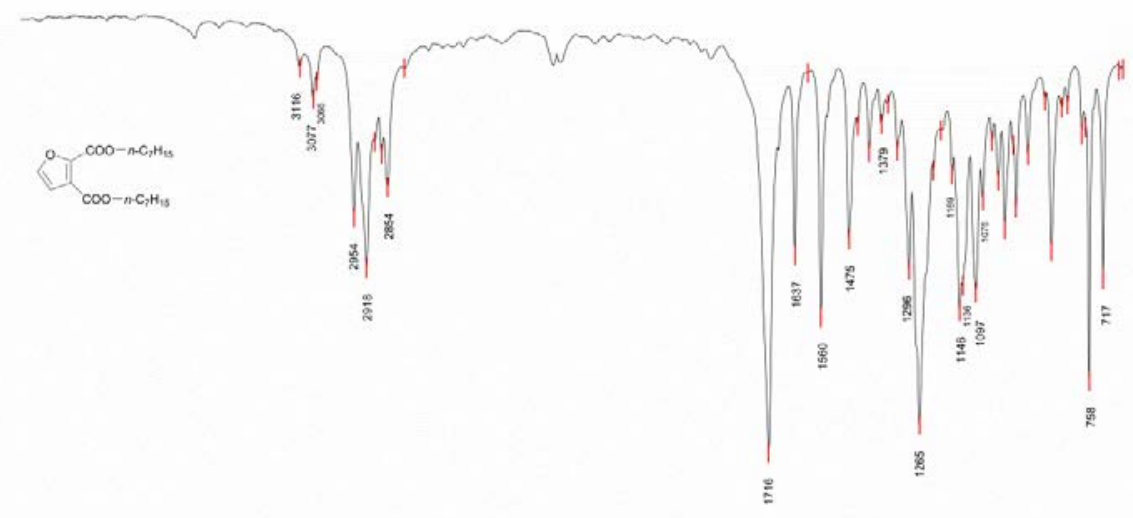

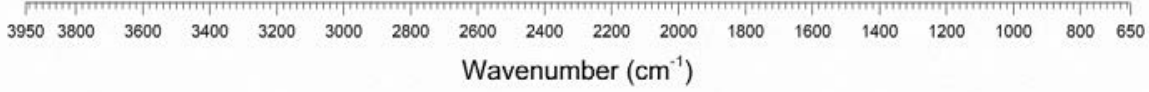

Figure S48. Infrared spectrum of diheptyl furan-2,3-dicarboxylate

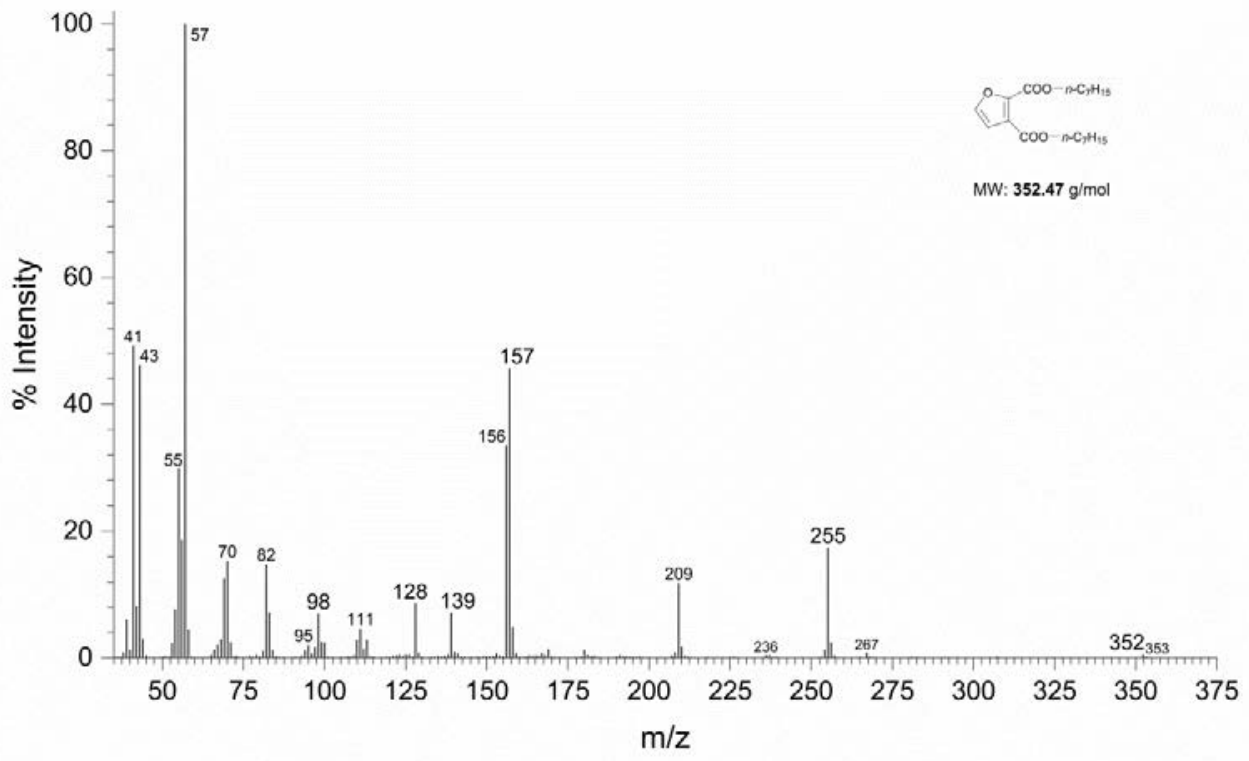

Figure S49. Mass spectrum of diheptyl furan-2,3-dicarboxylate 
E - DIOCTYL FURAN-2,3-DICARBOXYLATE
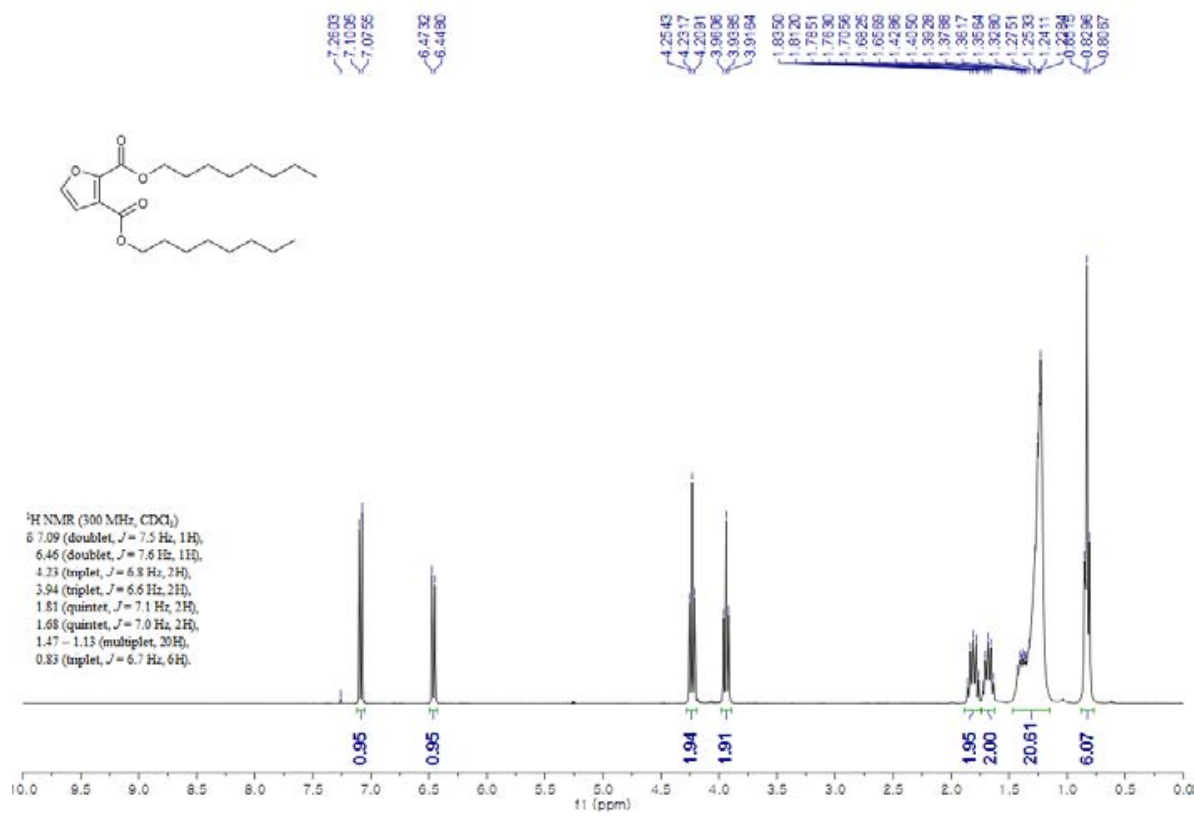

Figure S50. ${ }^{1} \mathrm{H}-\mathrm{NMR}$ spectrum of dioctyl furan-2,3-dicarboxylate

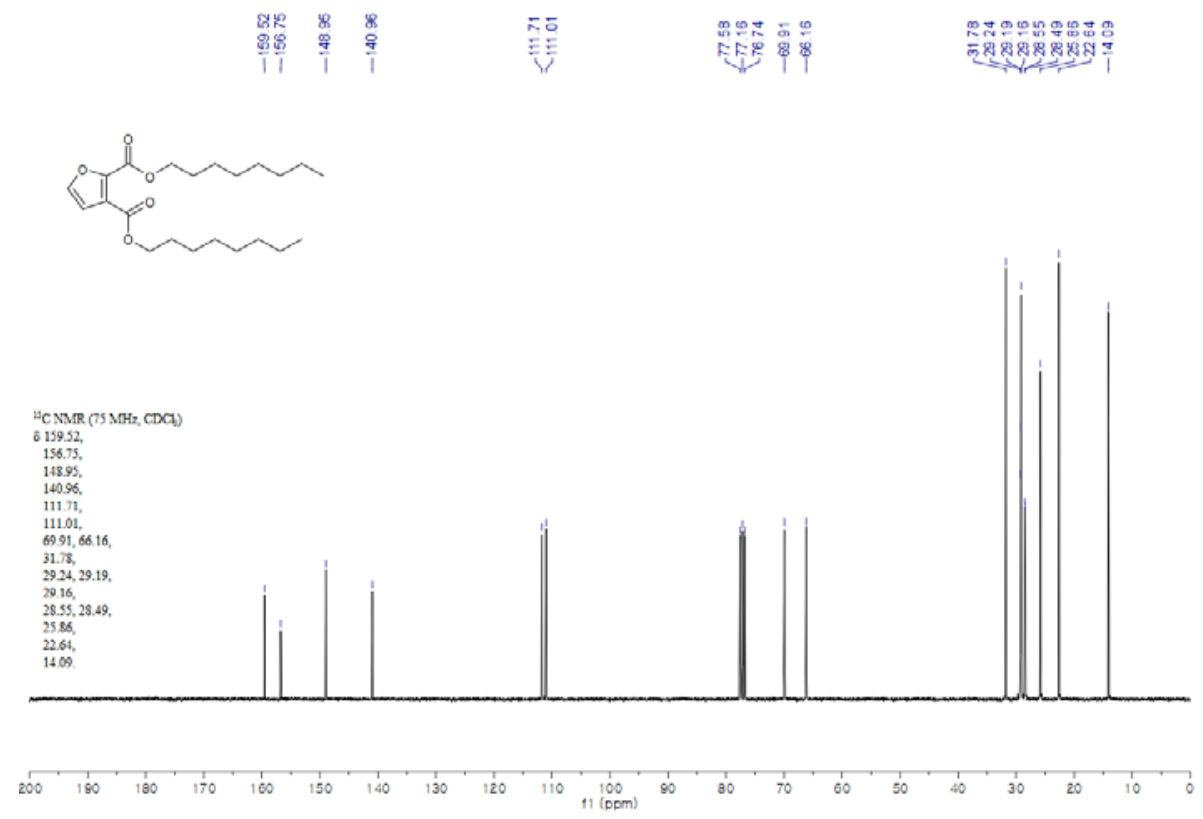

Figure S51. ${ }^{13} \mathrm{C}-\mathrm{NMR}$ spectrum of dioctyl furan-2,3-dicarboxylate 

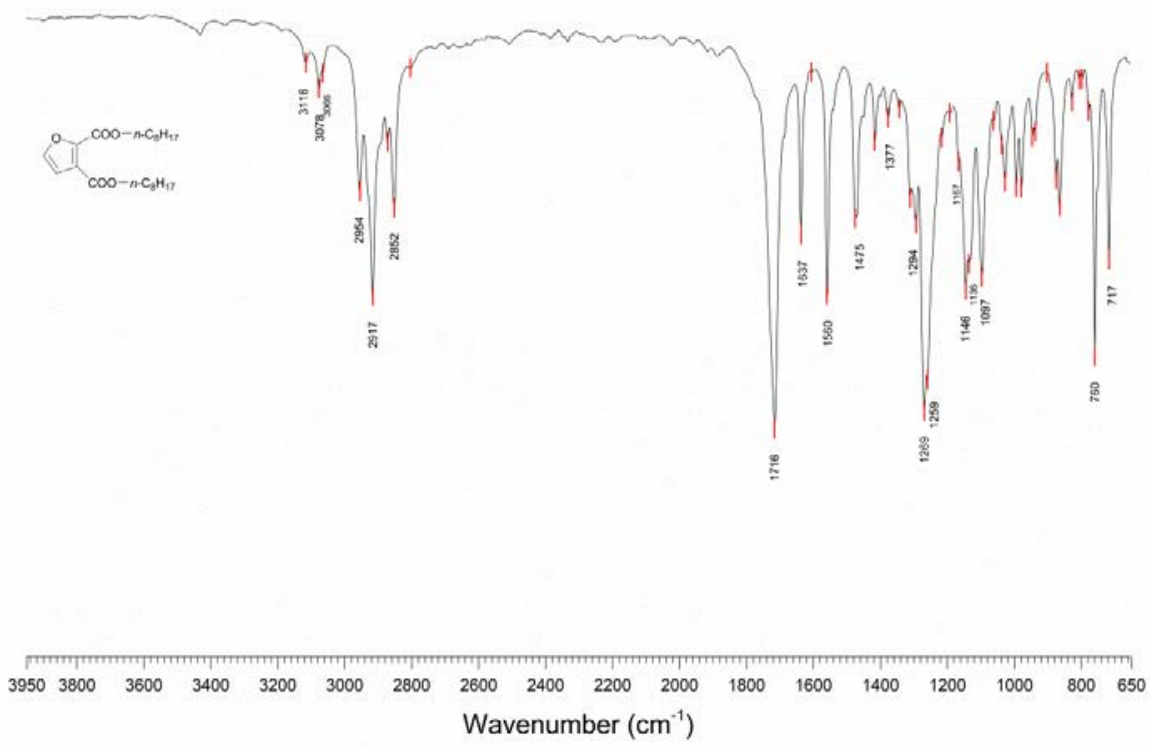

Figure S52. Infrared spectrum of dioctyl furan-2,3-dicarboxylate

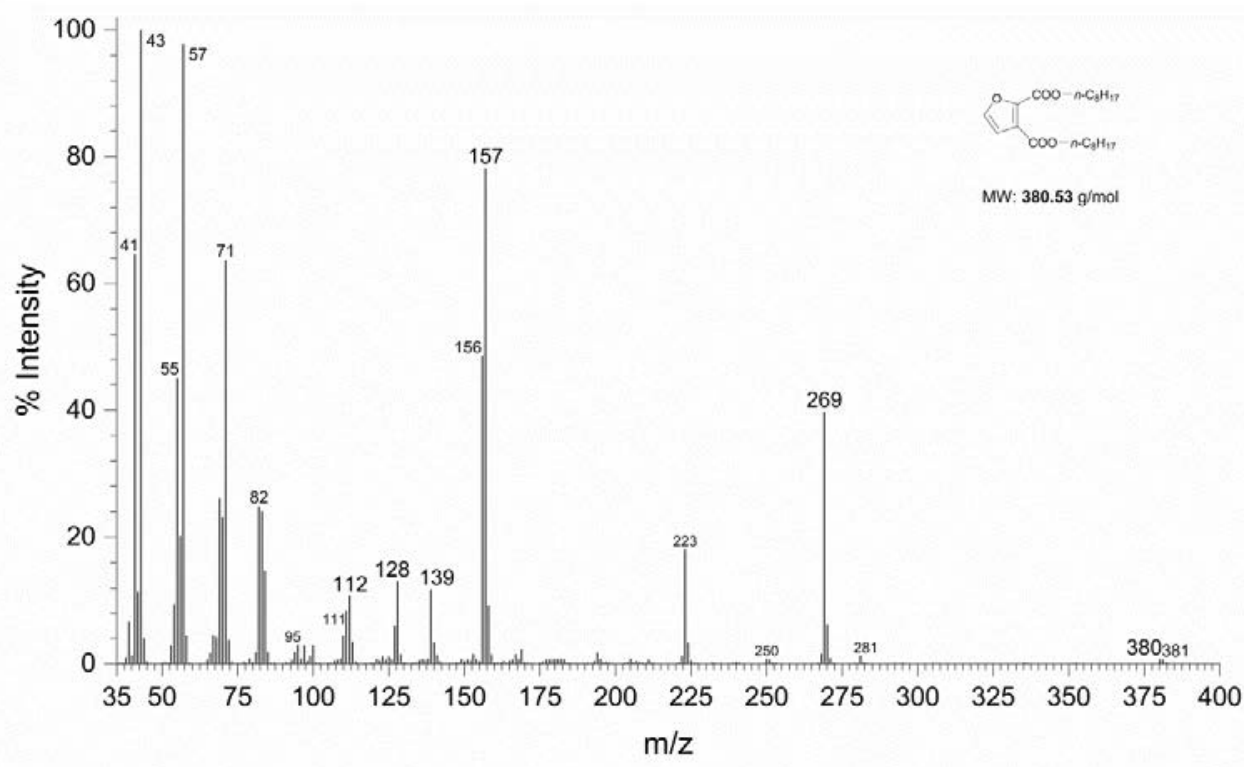

Figure S53. Mass spectrum of dioctyl furan-2,3-dicarboxylate 


\section{F - DIISOAMYL FURAN-2,3-DICARBOXYLATE (2,3-DIAF)}
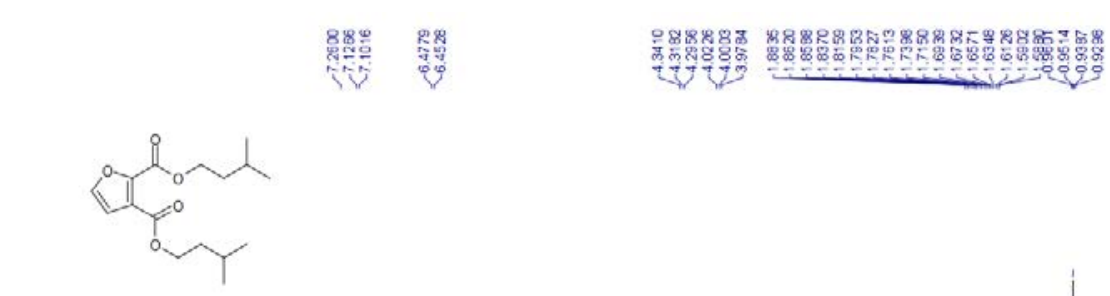

57.

67.11 (doutlet,,$=7.5 \mathrm{~Hz}$, Lit)

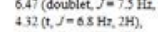

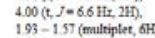

$193-157$ (miltiplet, 6H),
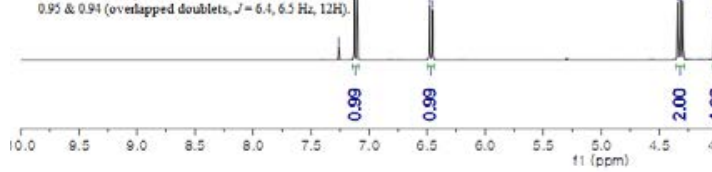

8 \&

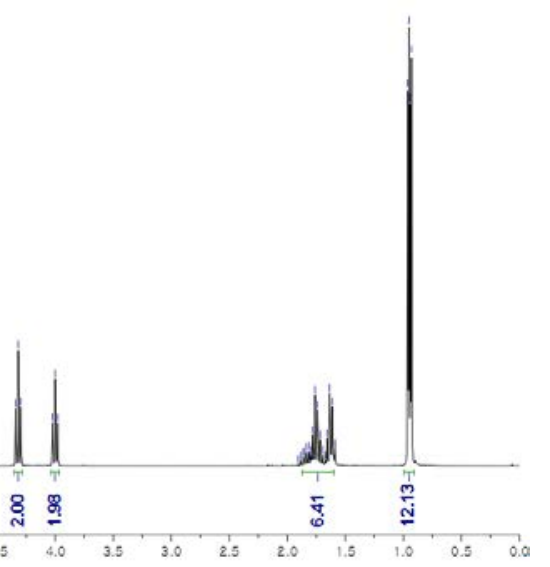

Figure S54. ${ }^{1} \mathrm{H}-\mathrm{NMR}$ spectrum of diisoamyl furan-2,3-dicarboxylate (2,3-DIAF)

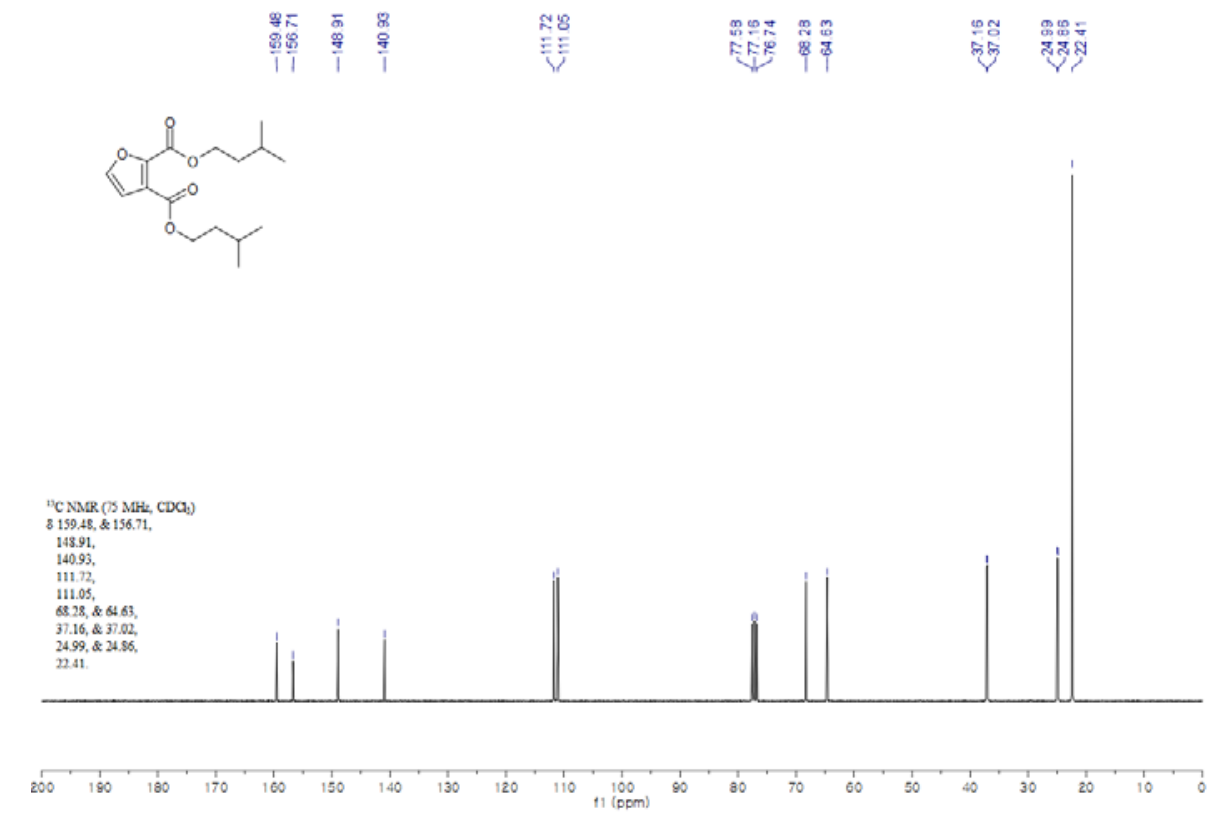

Figure S55. ${ }^{13} \mathrm{C}$-NMR spectrum of diisoamyl furan-2,3-dicarboxylate (2,3-DIAF) 

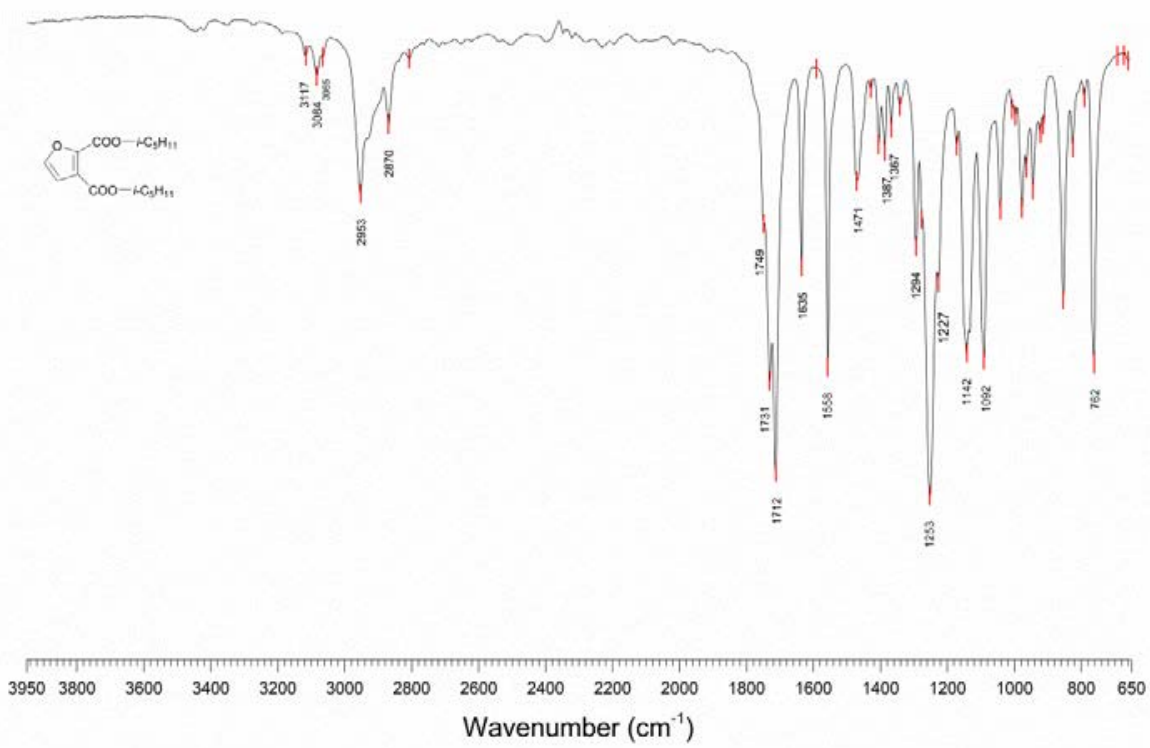

Figure S56. Infrared spectrum of diisoamyl furan-2,3-dicarboxylate (2,3-DIAF)

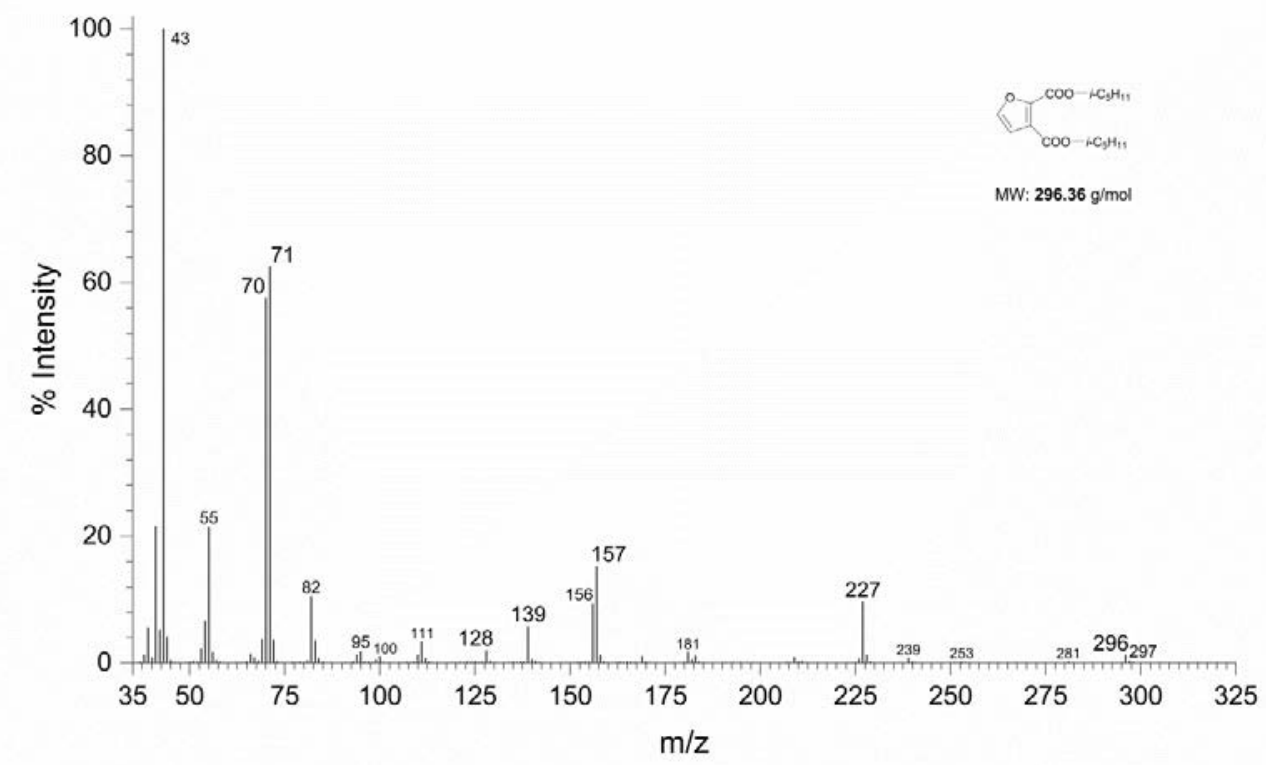

Figure S57. Mass spectrum of diisoamyl furan-2,3-dicarboxylate (2,3-DIAF) 


\section{G - DI(2-ETHYLHEXYL) FURAN-2,3-DICARBOXYLATE}

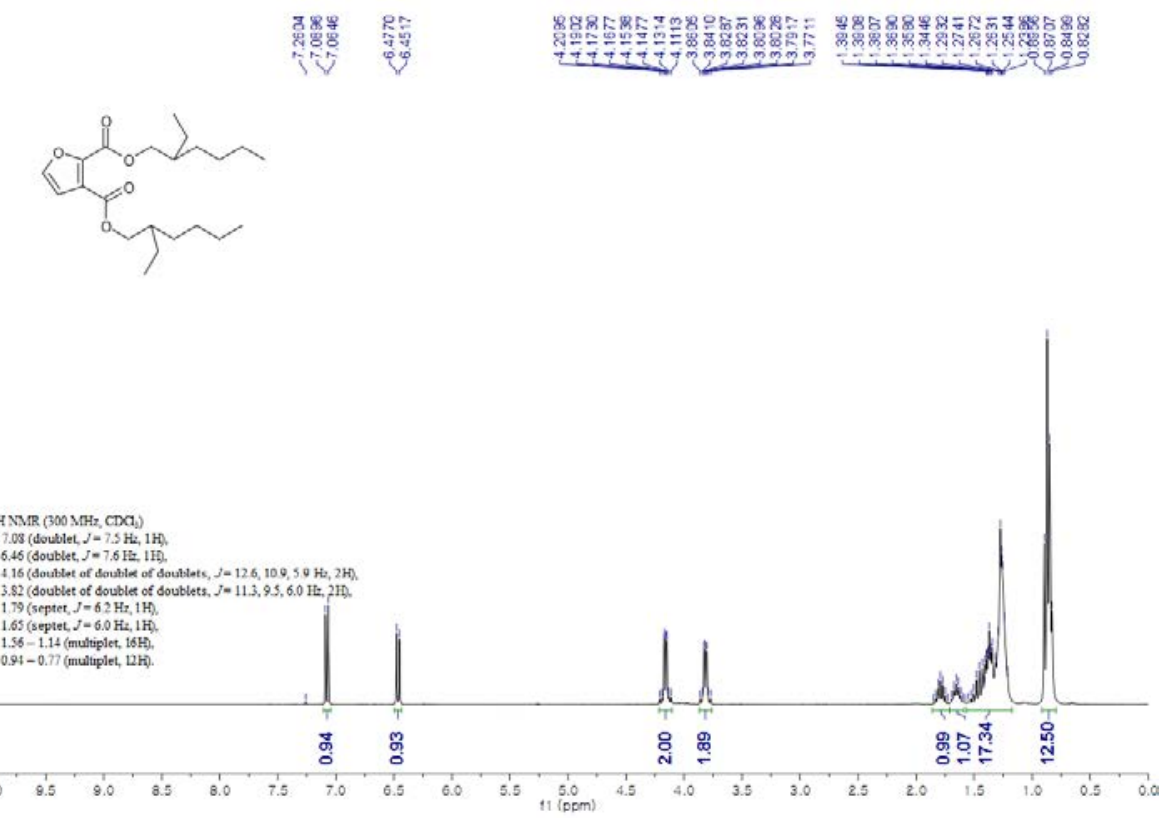

Figure S58. ${ }^{1} \mathrm{H}$-NMR spectrum of di(2-ethylhexyl) furan-2,3-dicarboxylate

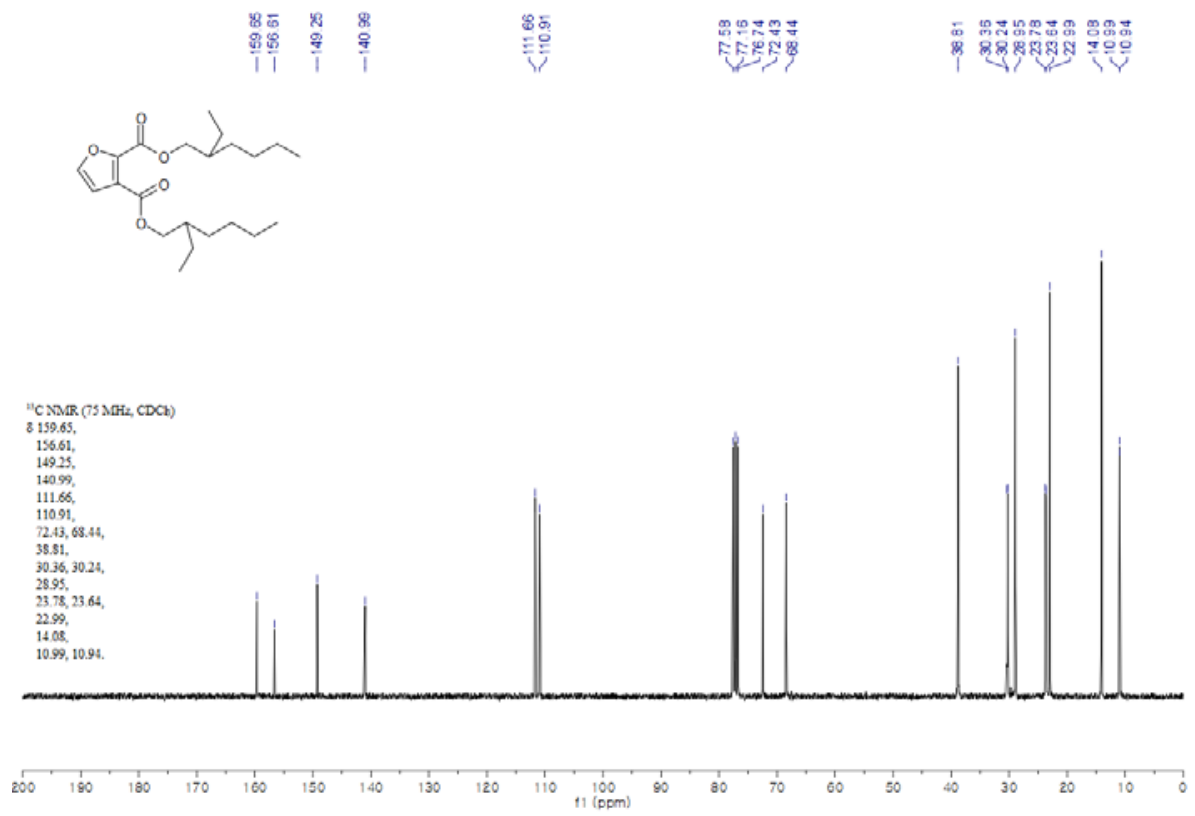

Figure S59. ${ }^{13}$ C-NMR spectrum of di(2-ethylhexyl) furan-2,3-dicarboxylate 

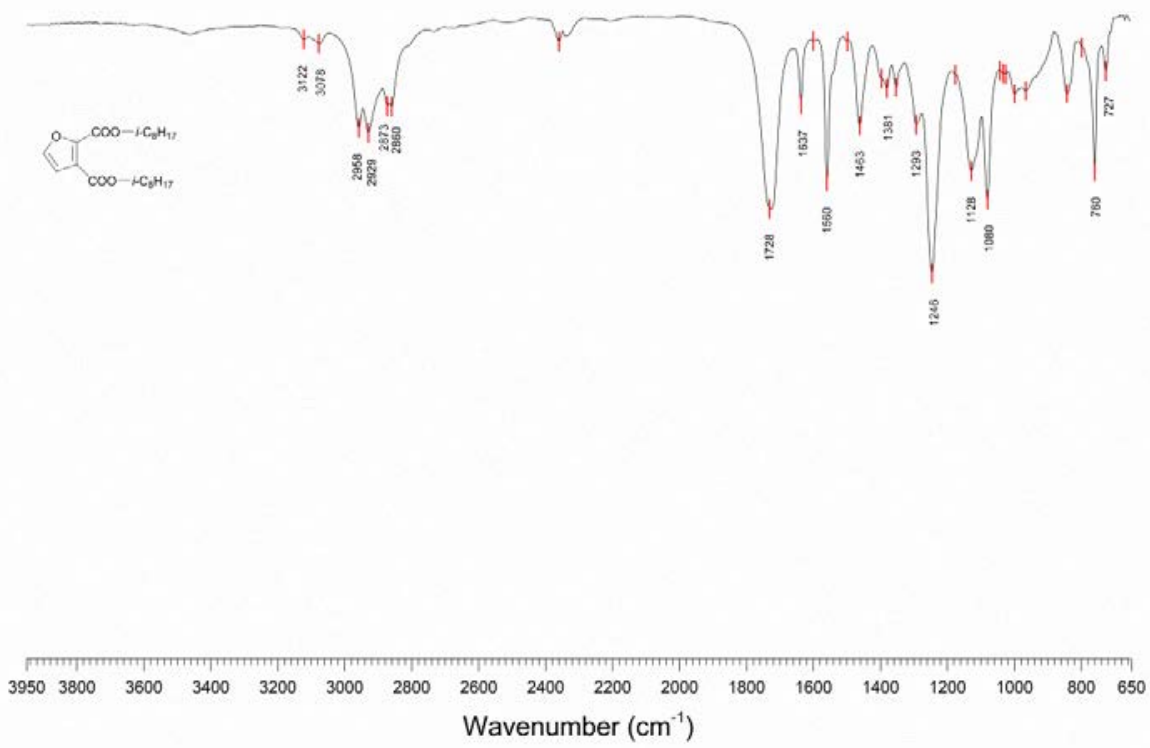

Figure S60. Infrared spectrum of di(2-ethylhexyl) furan-2,3-dicarboxylate

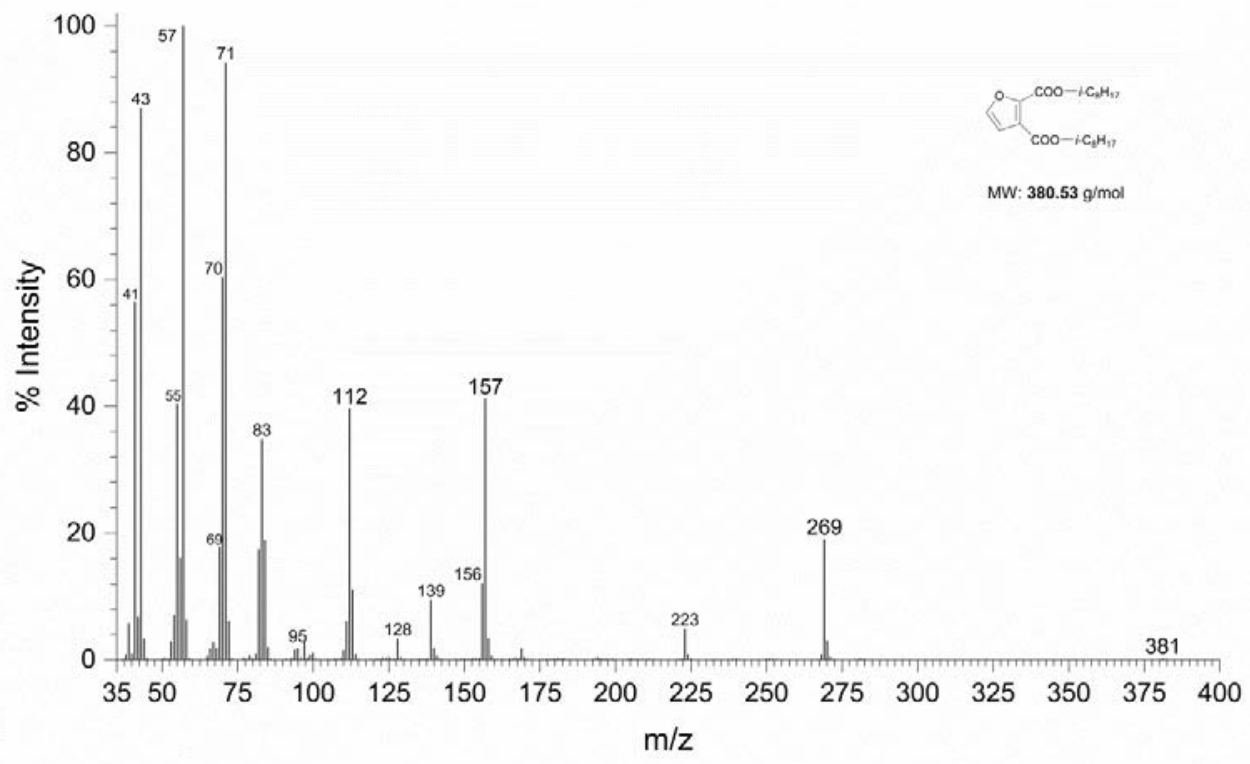

Figure S61. Mass spectrum of di(2-ethylhexyl) furan-2,3-dicarboxylate 


\section{Others}

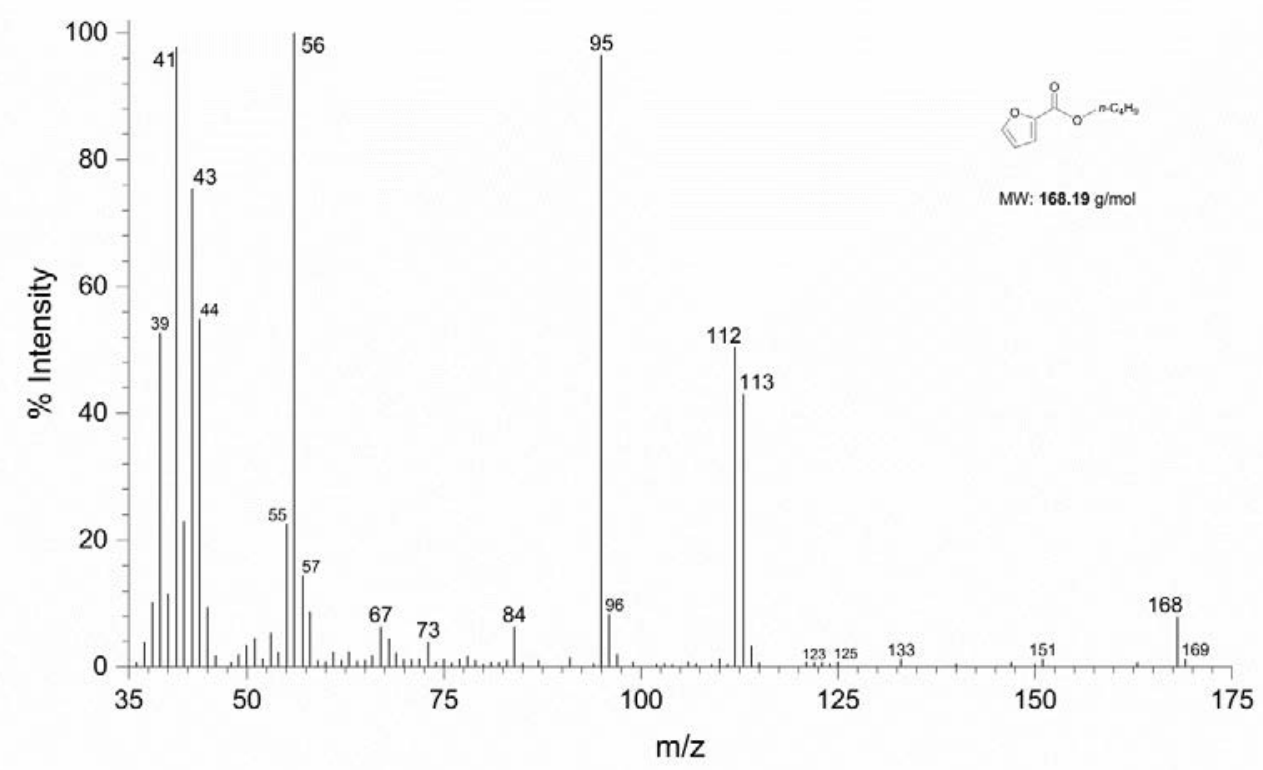

Figure S62. Mass spectrum of butyl 2-furoate

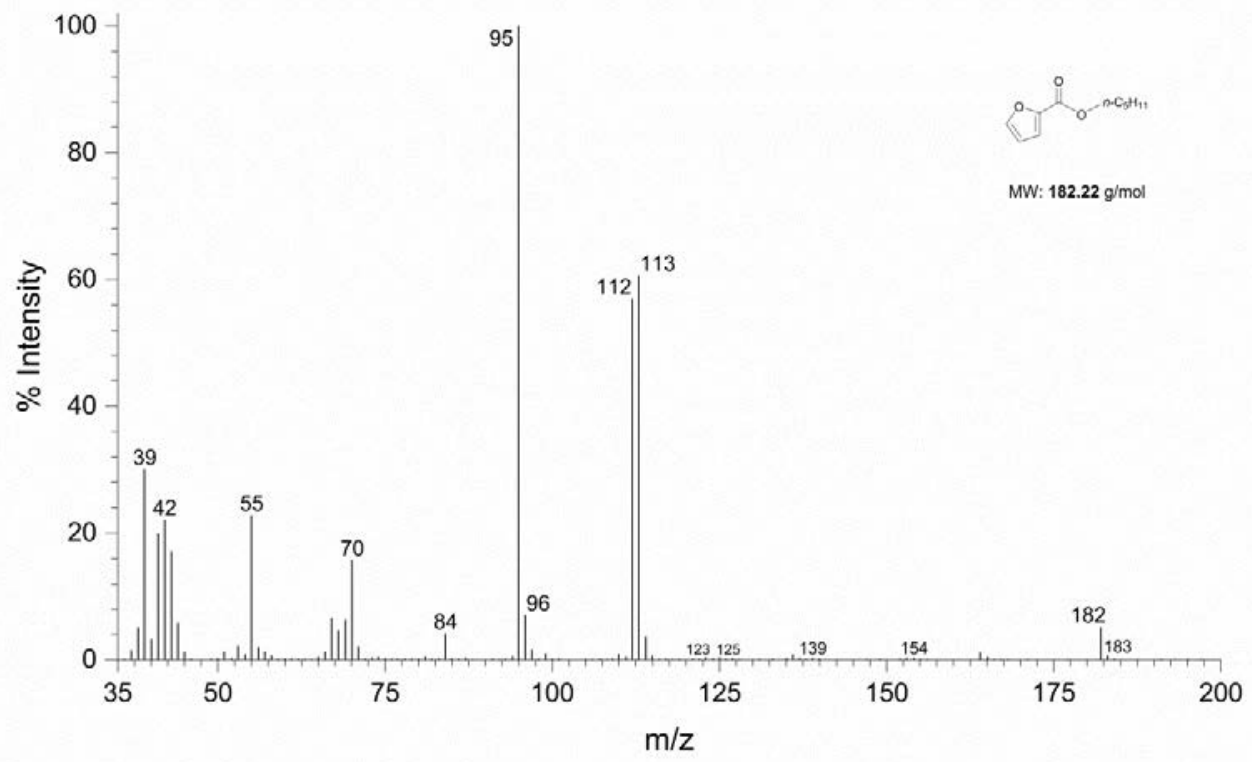

Figure S63. Mass spectrum of pentyl 2-furoate 


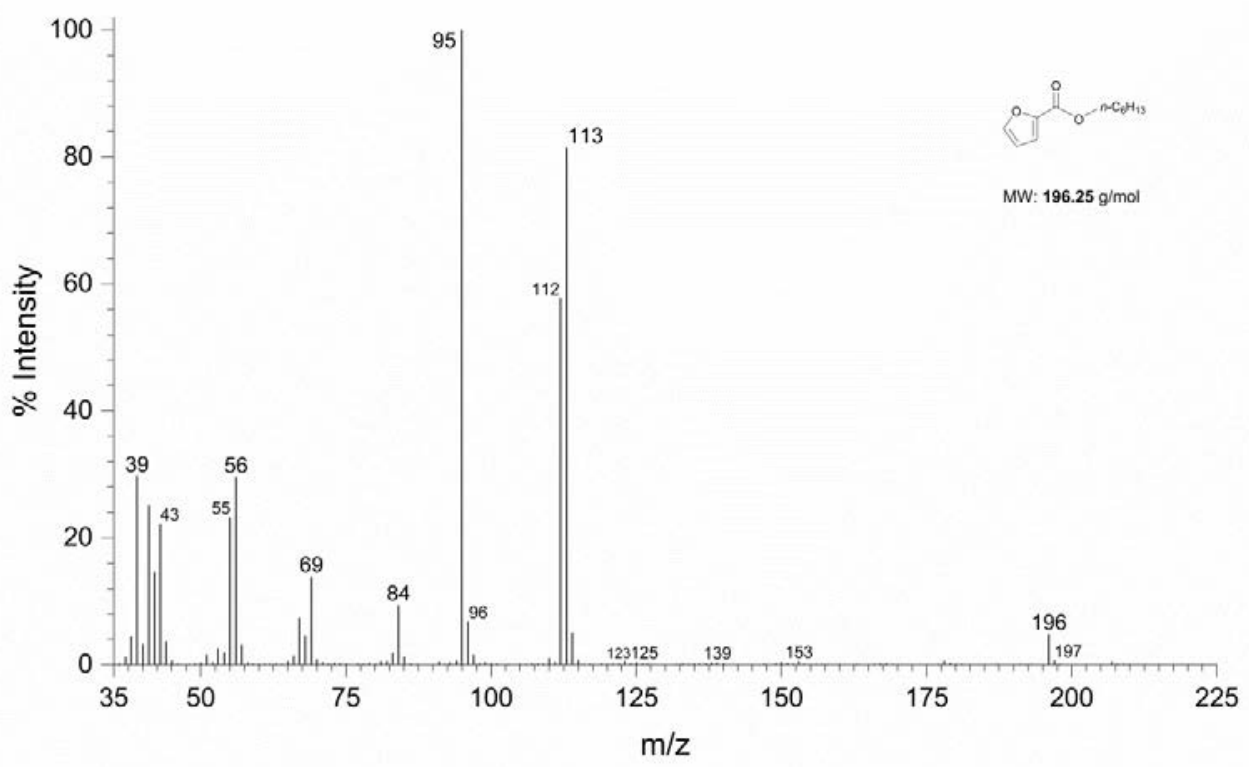

Figure S64. Mass spectrum of hexyl 2-furoate

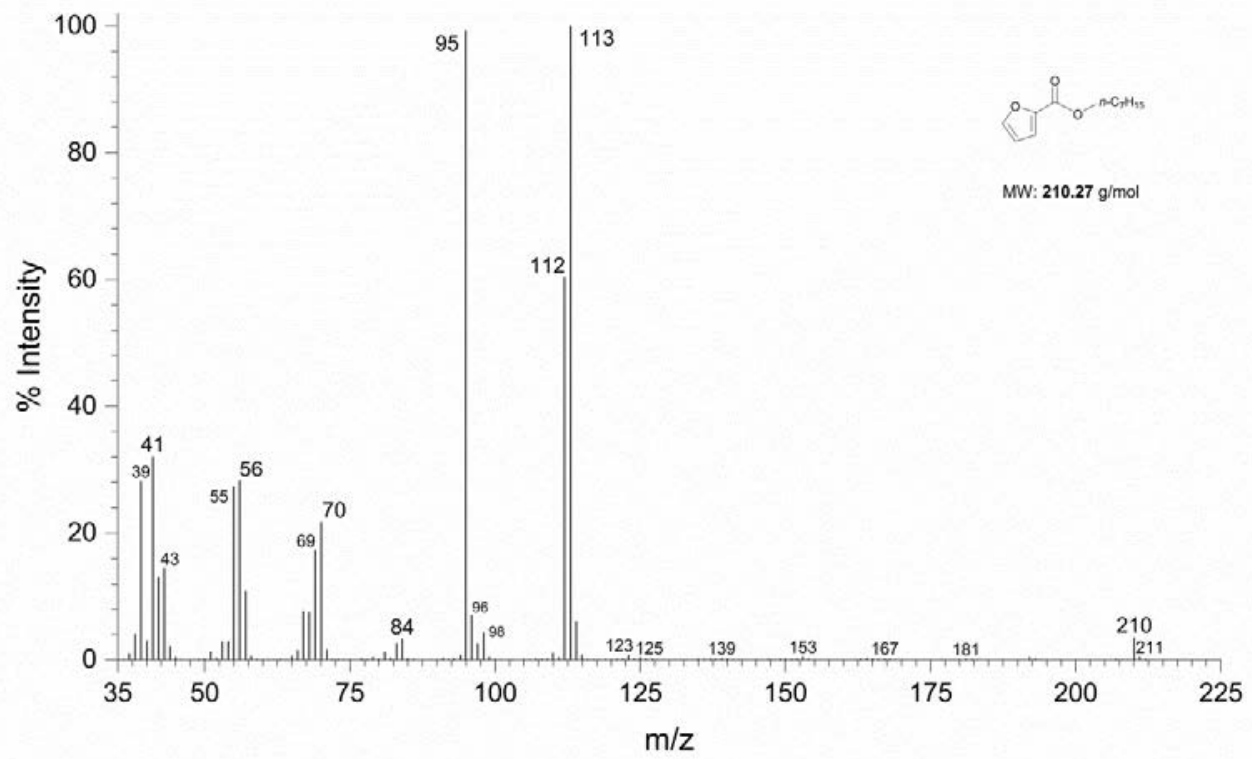

Figure S65. Mass spectrum of heptyl 2-furoate 


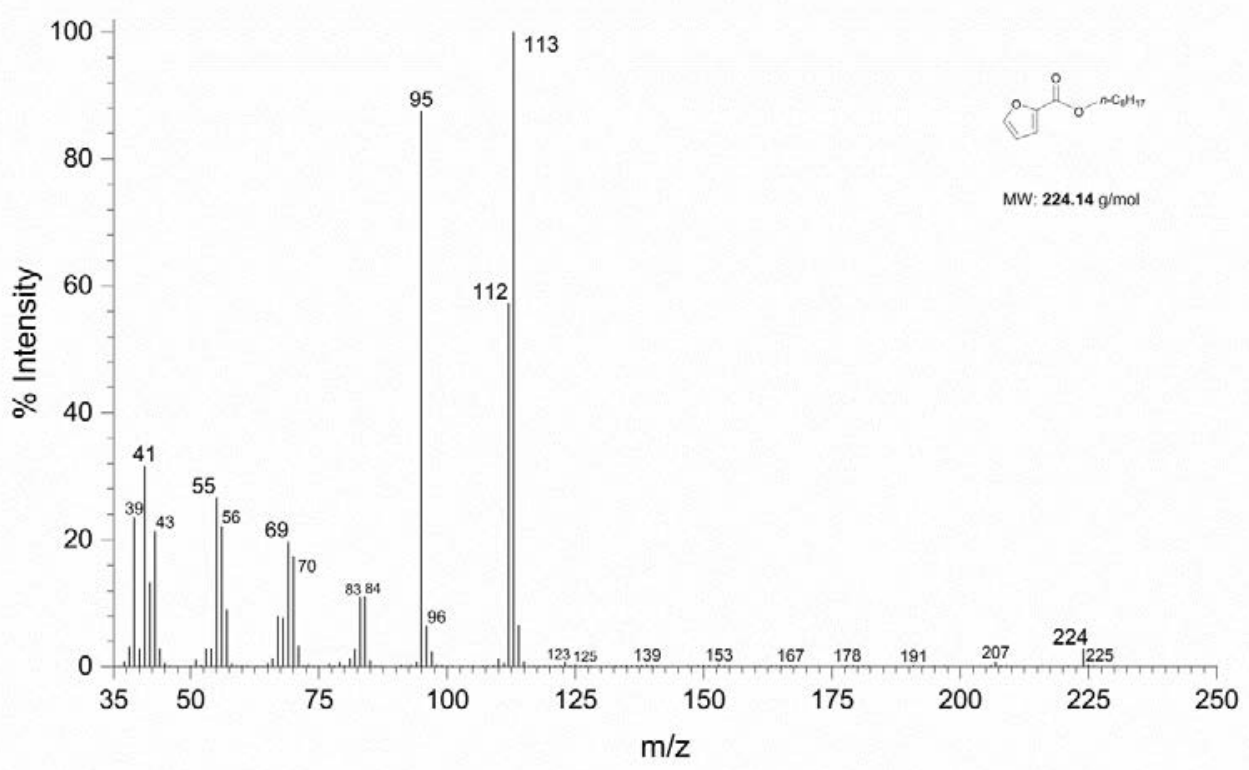

Figure S66. Mass spectrum of octyl 2-furoate

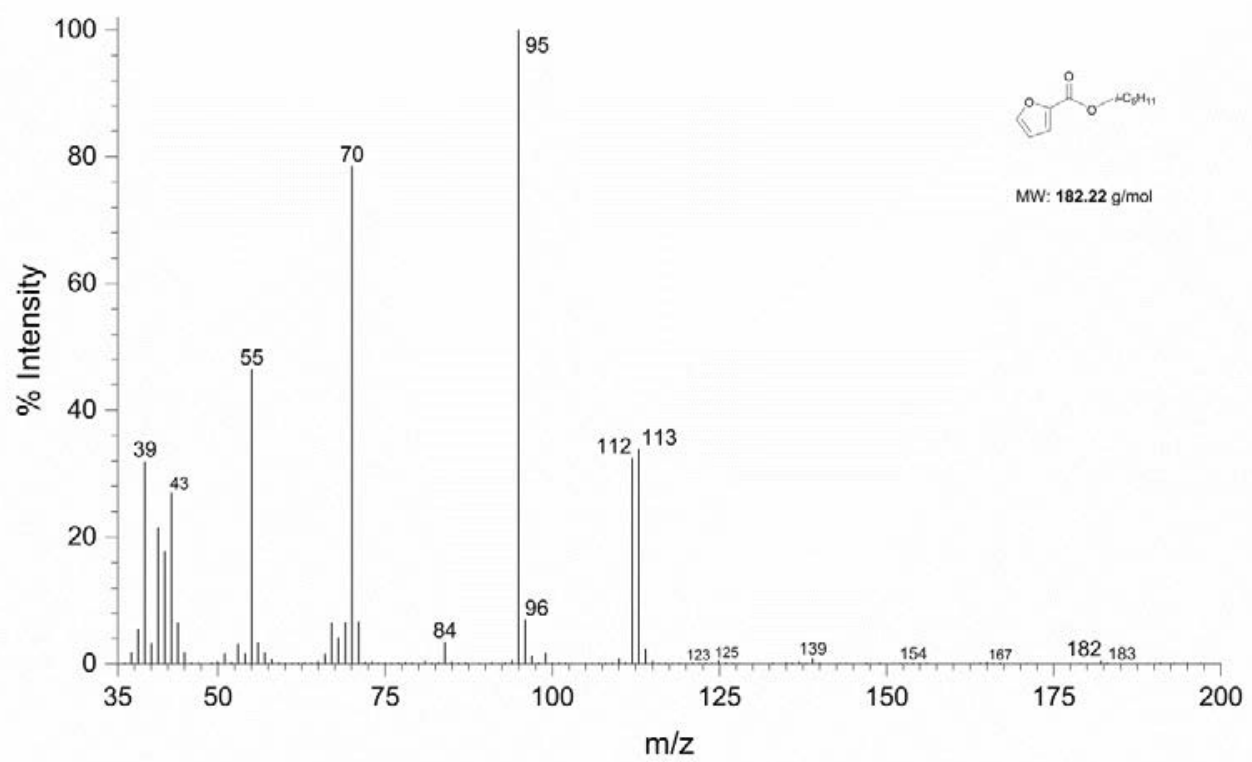

Figure S67. Mass spectrum of isoamyl 2-furoate 


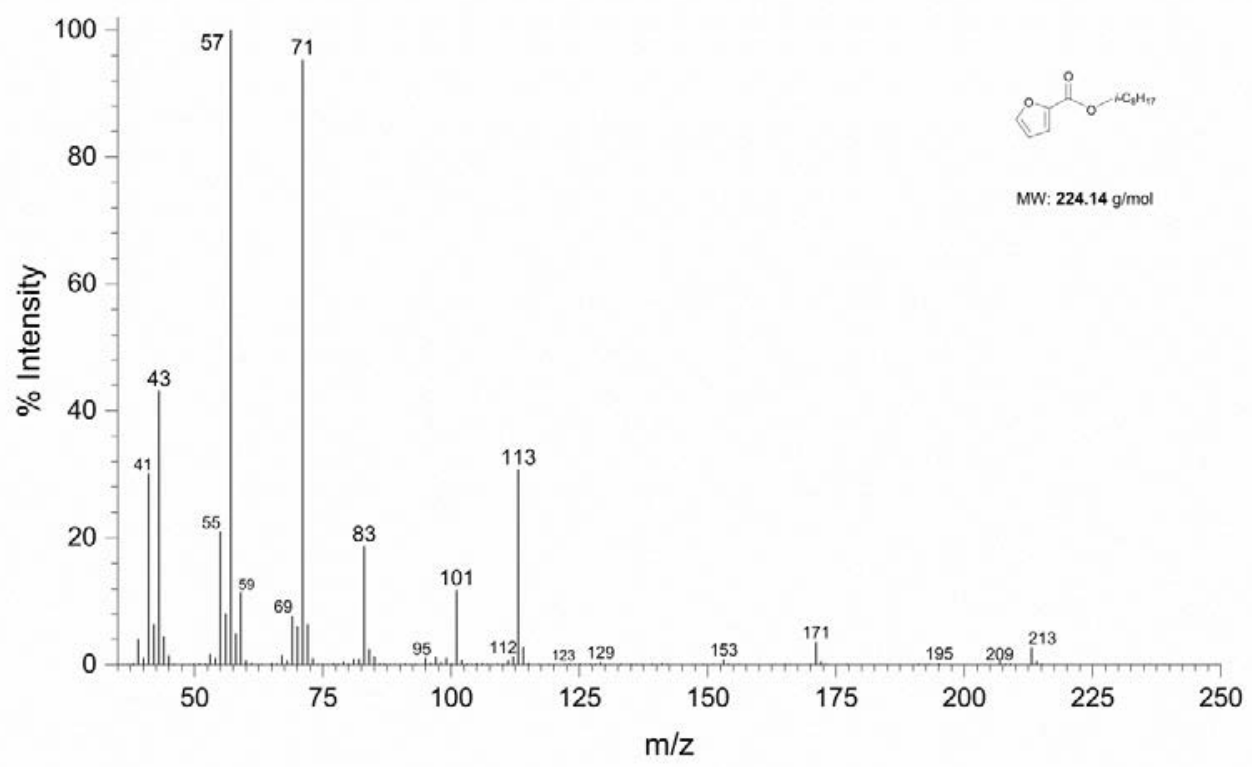

Figure S68. Mass spectrum of 2-ethylhexyl 2-furoate 


\section{PREPARATION OF PLASTICIZED FILMS}

Together with DOP, four lab-synthetic furanic compounds, namely di-n-butyl furan-2,5dicarboxylate (2,5-DBF), di-n-butyl furan-2,3-dicarboxylate (2,3-DBF), diisoamyl furan-2,5dicarboxylate (2,5-DIAF), and diisoamyl furan-2,3-dicarboxylate (2,3-DIAF) acted as plasticizer and were combined with poly(vinyl chloride) (PVC) matrix into plastic films. Tetrahydrofuran (THF, anhydrous and inhibitor-free, over 99.9\%), PVC (powder, average $\mathrm{M}_{\mathrm{w}} \sim 80,000$, average $\left.\mathrm{M}_{\mathrm{n}} \sim 47,000\right)$ were purchased from Sigma-Aldrich and were used directly when received. Bis(2ethylhexyl) phthalate (DOP, over 98 \%) was purchased from TCI Chemicals (Japan), which was used as reference plasticizer chemical.

PVC powder (2.5 g) was dissolved together with plasticizers (PLS) in ca. $55 \mathrm{~mL}$ of THF, and was stirred for at least $3 \mathrm{~h}$. Homogenous solution was then cast on a clean, dustless petri dish. Solvent was allowed to free evaporate overnight to obtain flexible films. The flexible films continued to be put in a vacuum oven at $35^{\circ} \mathrm{C}$ for $24 \mathrm{~h}$ more. The terms of "PLS xx-phr" represent xx parts by weight PLS per 100 parts by weight of PVC powder. 
Table S8. Formulations for preparation of PVC-PLS films

\begin{tabular}{|c|c|c|c|c|}
\hline & $\begin{array}{l}\text { Weight } \\
\text { of PLS (g) }\end{array}$ & $\begin{array}{l}\text { Weight } \\
\text { of PVC (g) }\end{array}$ & $\begin{array}{l}\text { Volume } \\
\text { of THF }\end{array}$ & $\begin{array}{c}\text { Plasticized films } \\
\text { designated as }\end{array}$ \\
\hline \multirow{6}{*}{ 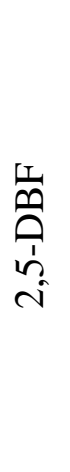 } & $0.1250 \pm 0.0005$ & \multirow{6}{*}{$\begin{array}{c}2.5000 \\
\pm 0.0005\end{array}$} & \multirow{6}{*}{$55 \mathrm{~mL}$} & 2,5-DBF 5.3phr \\
\hline & $0.2500 \pm 0.0005$ & & & 2,5-DBF 11.1phr \\
\hline & $0.5000 \pm 0.0005$ & & & 2,5-DBF 25phr \\
\hline & $0.7500 \pm 0.0005$ & & & 2,5-DBF 42.9phr \\
\hline & $1.0000 \pm 0.0005$ & & & 2,5-DBF 66.7phr \\
\hline & $1.2500 \pm 0.0005$ & & & 2,5-DBF 100phr \\
\hline \multirow{6}{*}{ 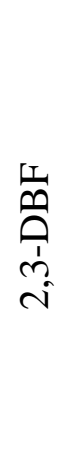 } & $0.1250 \pm 0.0005$ & \multirow{6}{*}{$\begin{array}{c}2.5000 \\
\pm 0.0005\end{array}$} & \multirow{6}{*}{$55 \mathrm{~mL}$} & 2,3-DBF 5.3phr \\
\hline & $0.2500 \pm 0.0005$ & & & 2,3-DBF 11.1phr \\
\hline & $0.5000 \pm 0.0005$ & & & 2,3-DBF 25phr \\
\hline & $0.7500 \pm 0.0005$ & & & 2,3-DBF 42.9phr \\
\hline & $1.0000 \pm 0.0005$ & & & 2,3-DBF 66.7phr \\
\hline & $1.2500 \pm 0.0005$ & & & 2,3-DBF 100phr \\
\hline \multirow{6}{*}{ 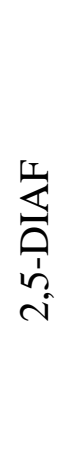 } & $0.1250 \pm 0.0005$ & \multirow{6}{*}{$\begin{array}{c}2.5000 \\
\pm 0.0005\end{array}$} & \multirow{6}{*}{$55 \mathrm{~mL}$} & 2,5-DIAF 5.3phr \\
\hline & $0.2500 \pm 0.0005$ & & & 2,5-DIAF 11.1phI \\
\hline & $0.5000 \pm 0.0005$ & & & 2,5-DIAF 25phr \\
\hline & $0.7500 \pm 0.0005$ & & & 2,5-DIAF 42.9phI \\
\hline & $1.0000 \pm 0.0005$ & & & 2,5-DIAF 66.7phr \\
\hline & $1.2500 \pm 0.0005$ & & & 2,5-DIAF 100phr \\
\hline \multirow{6}{*}{ 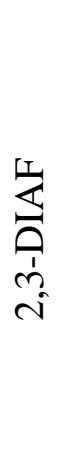 } & $0.1250 \pm 0.0005$ & \multirow{6}{*}{$\begin{array}{c}2.5000 \\
\pm 0.0005\end{array}$} & \multirow{6}{*}{$55 \mathrm{~mL}$} & 2,3-DIAF 5.3phr \\
\hline & $0.2500 \pm 0.0005$ & & & 2,3-DIAF 11.1phr \\
\hline & $0.5000 \pm 0.0005$ & & & 2,3-DIAF 25phr \\
\hline & $0.7500 \pm 0.0005$ & & & 2,3-DIAF 42.9phı \\
\hline & $1.0000 \pm 0.0005$ & & & 2,3-DIAF 66.7phr \\
\hline & $1.2500 \pm 0.0005$ & & & 2,3-DIAF 100phr \\
\hline
\end{tabular}




\begin{tabular}{|c|c|c|c|c|}
\hline \multirow{6}{*}{ ڤิ } & $0.1250 \pm 0.0005$ & \multirow{6}{*}{$\begin{array}{r}2.5000 \\
\pm 0.0005\end{array}$} & \multirow{6}{*}{$55 \mathrm{~mL}$} & DOP 5.3phr \\
\hline & $0.2500 \pm 0.0005$ & & & DOP 11.1phr \\
\hline & $0.5000 \pm 0.0005$ & & & DOP 25phr \\
\hline & $0.7500 \pm 0.0005$ & & & DOP 42.9phr \\
\hline & $1.0000 \pm 0.0005$ & & & DOP 66.7phr \\
\hline & $1.2500 \pm 0.0005$ & & & DOP 100phr \\
\hline
\end{tabular}




\section{SURFACE MORPHOLOGY}

\section{Di(2-ethylhexyl) phthalate (DOP)}

Images were captured at $2 \mathrm{kV}$, x3000 magnification. There was no obvious phase separation between plasticizer and the matrix.

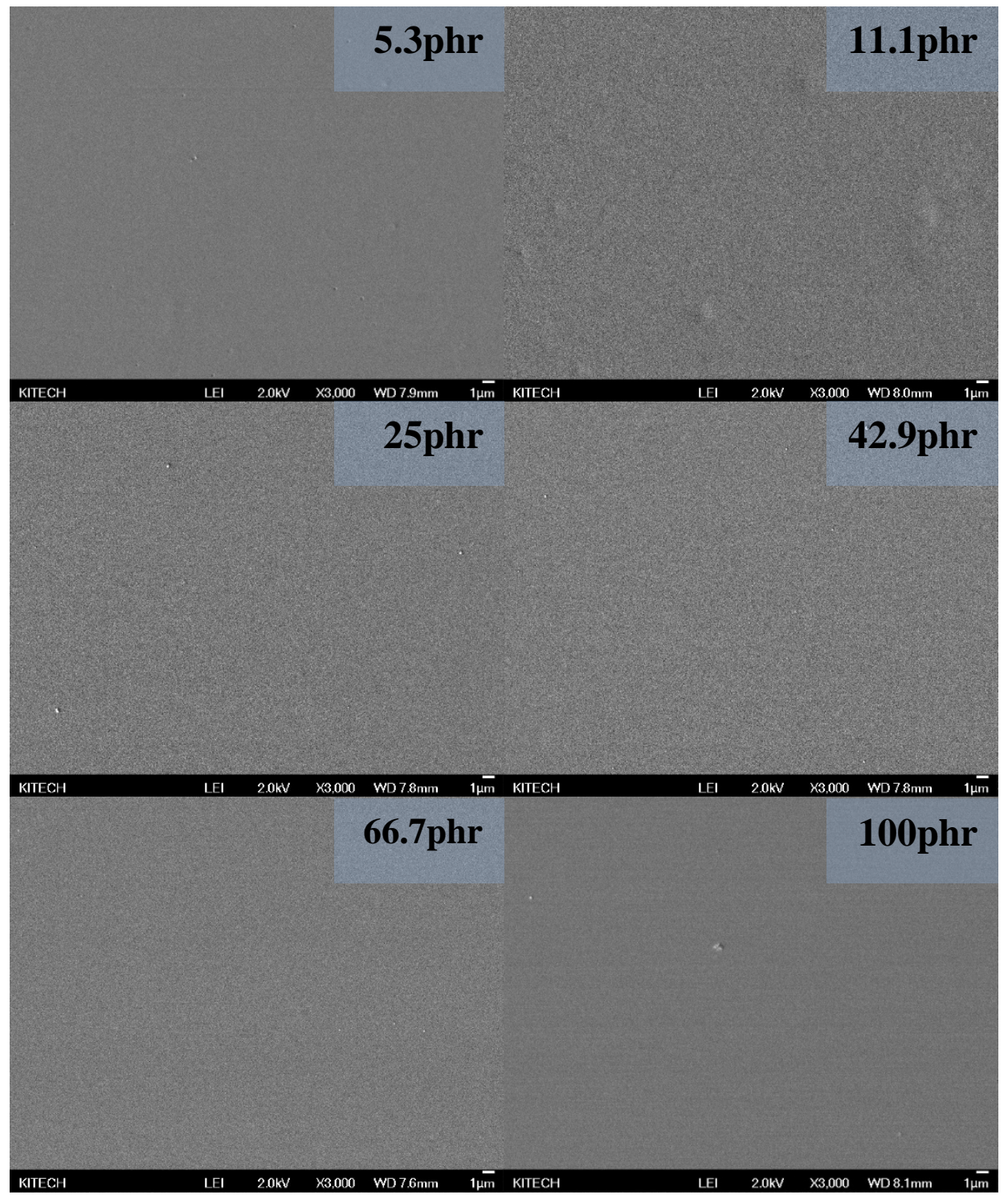

Figure S69. SEM image set of PVC-DOP films 


\section{Di-n-butyl furan-2,5-dicarboxylate (2,5-DBF)}

Images were captured at $2 \mathrm{kV}$, x3000 magnification. There was no obvious phase separation between plasticizer and the matrix.

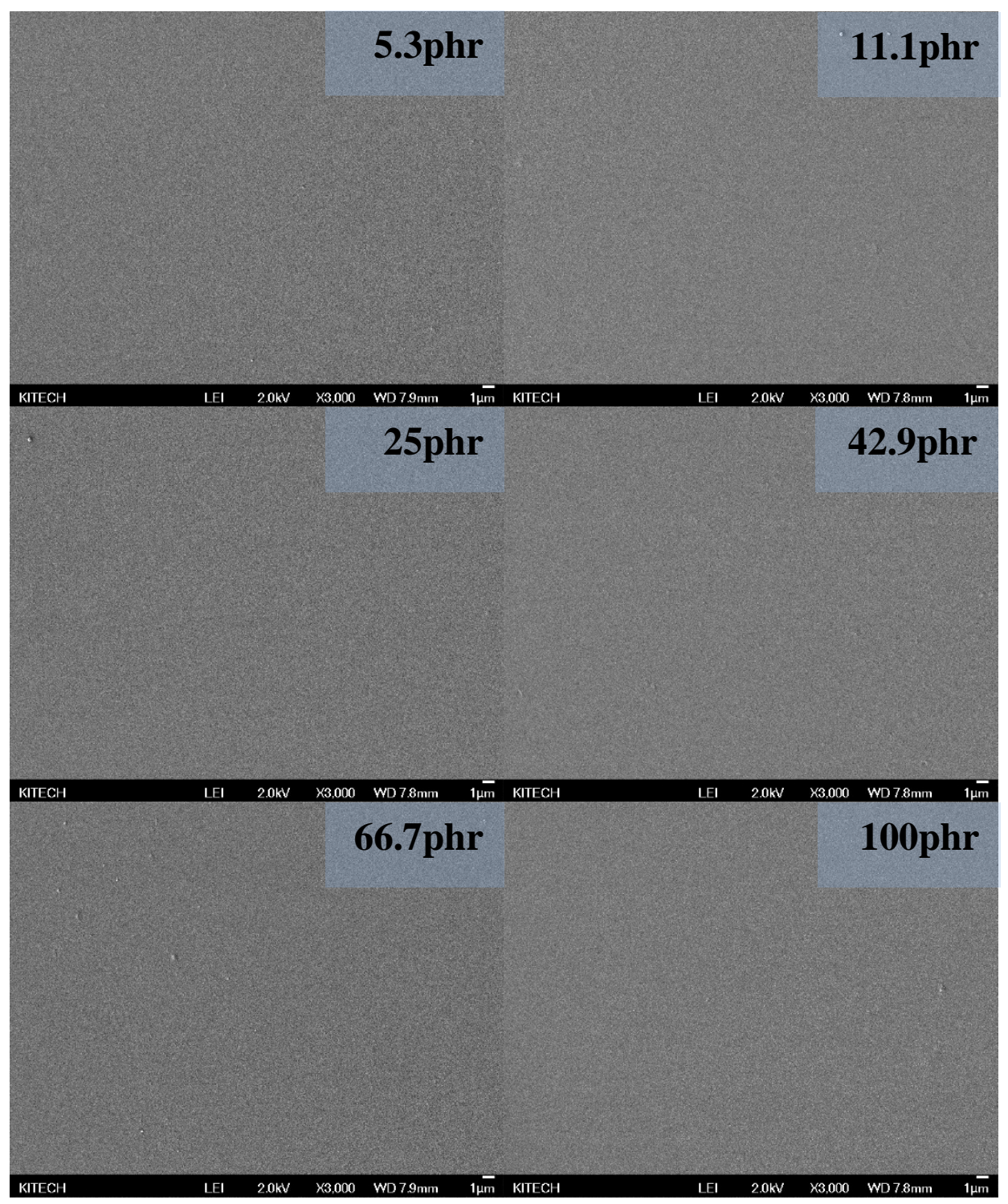

Figure S70. SEM image set of PVC-2,5-DBF films 


\section{Diisoamyl furan-2,5-dicarboxylate (2,5-DIAF)}

Images were captured at $2 \mathrm{kV}$, x3000 magnification. There was no obvious phase separation between plasticizer and the matrix.

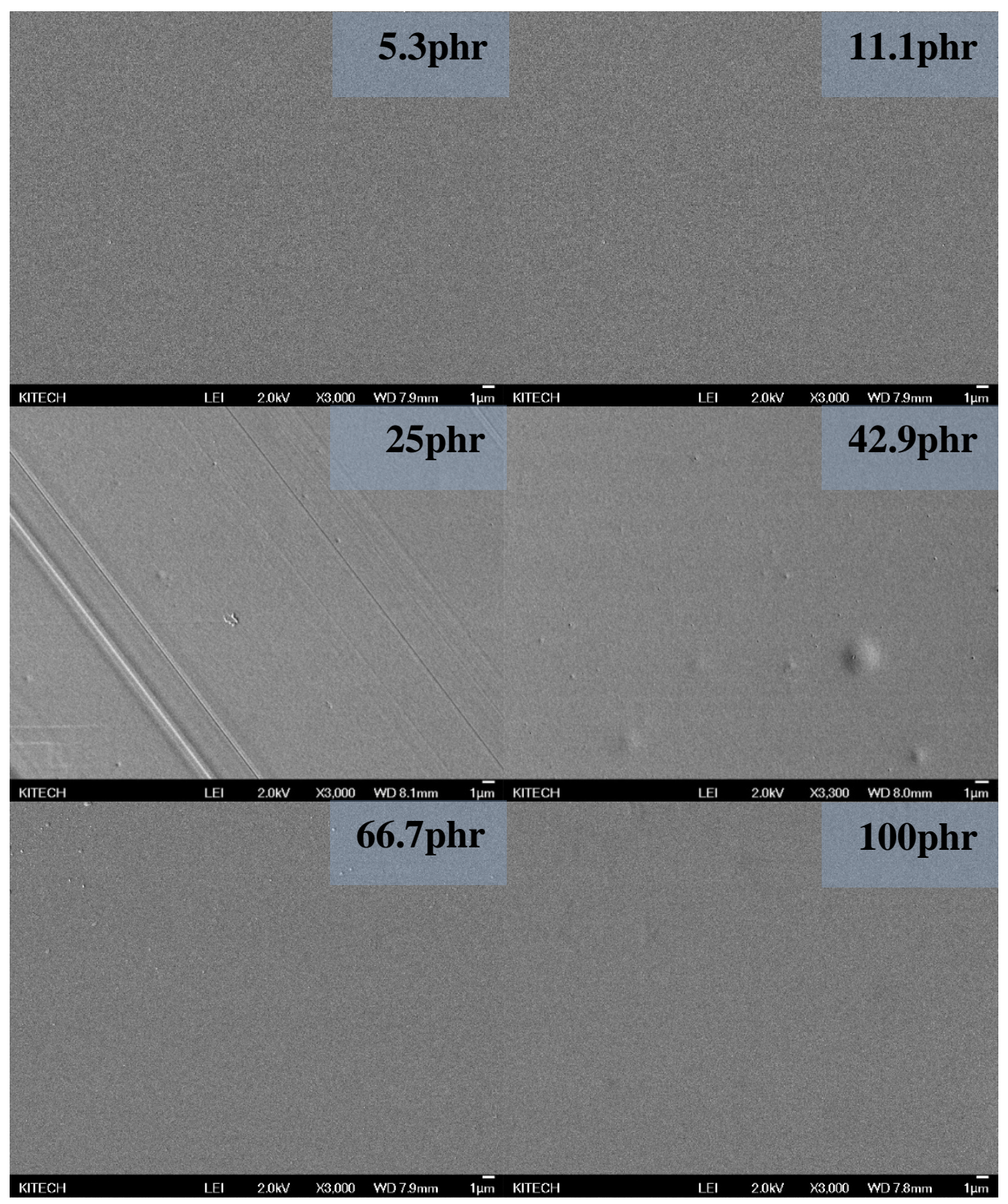

Figure S71. SEM image set of PVC-2,5-DIAF films 


\section{Di-n-butyl furan-2,3-dicarboxylate (2,3-DBF)}

Images were captured at $2 \mathrm{kV}$, x3000 magnification. There was no obvious phase separation between plasticizer and the matrix.

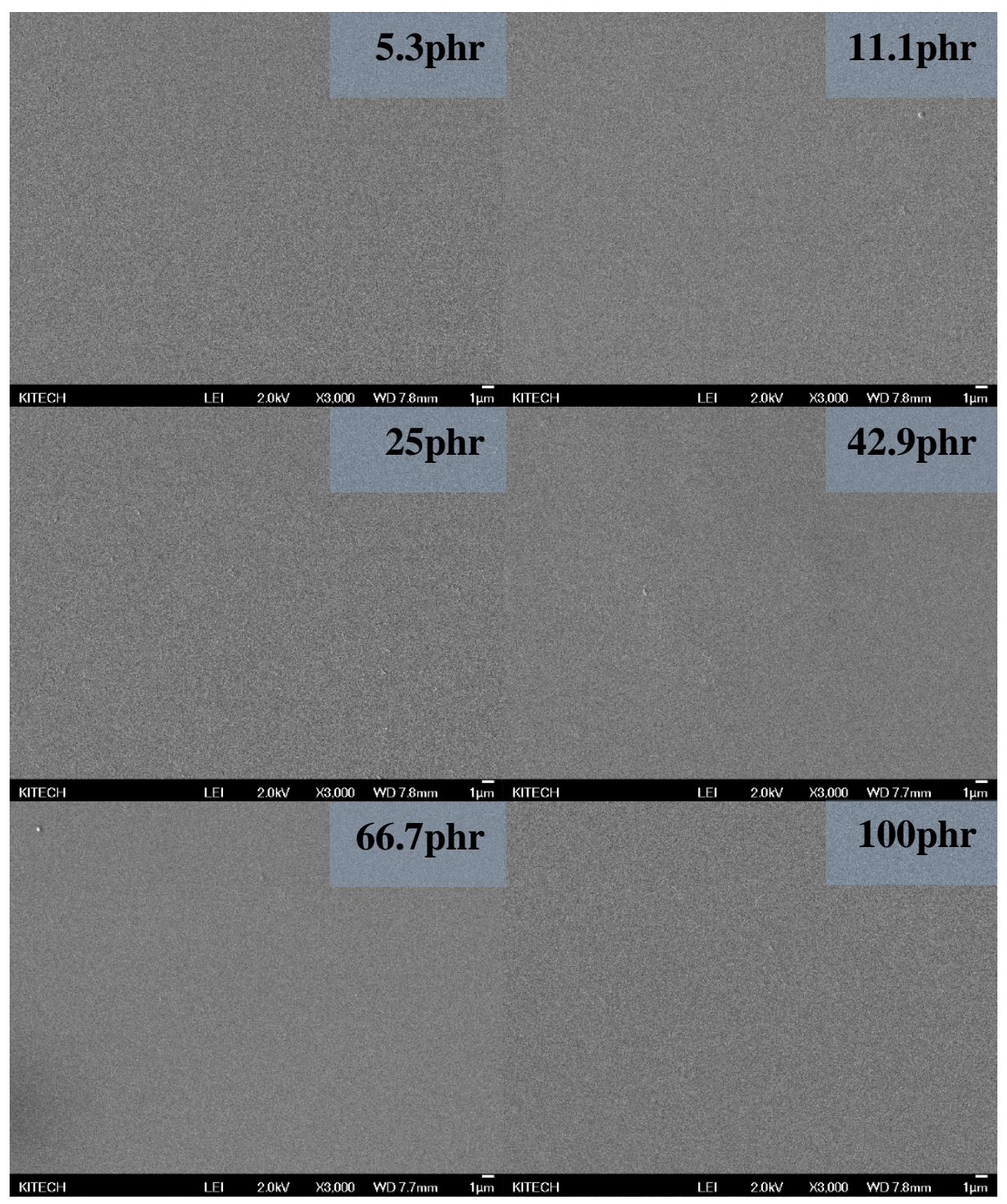

Figure S72. SEM image set of PVC-2,3-DBF films 


\section{Diisoamyl furan-2,3-dicarboxylate (2,3-DIAF)}

Images were captured at $2 \mathrm{kV}$, x3000 magnification. There was no obvious phase separation between plasticizer and the matrix.

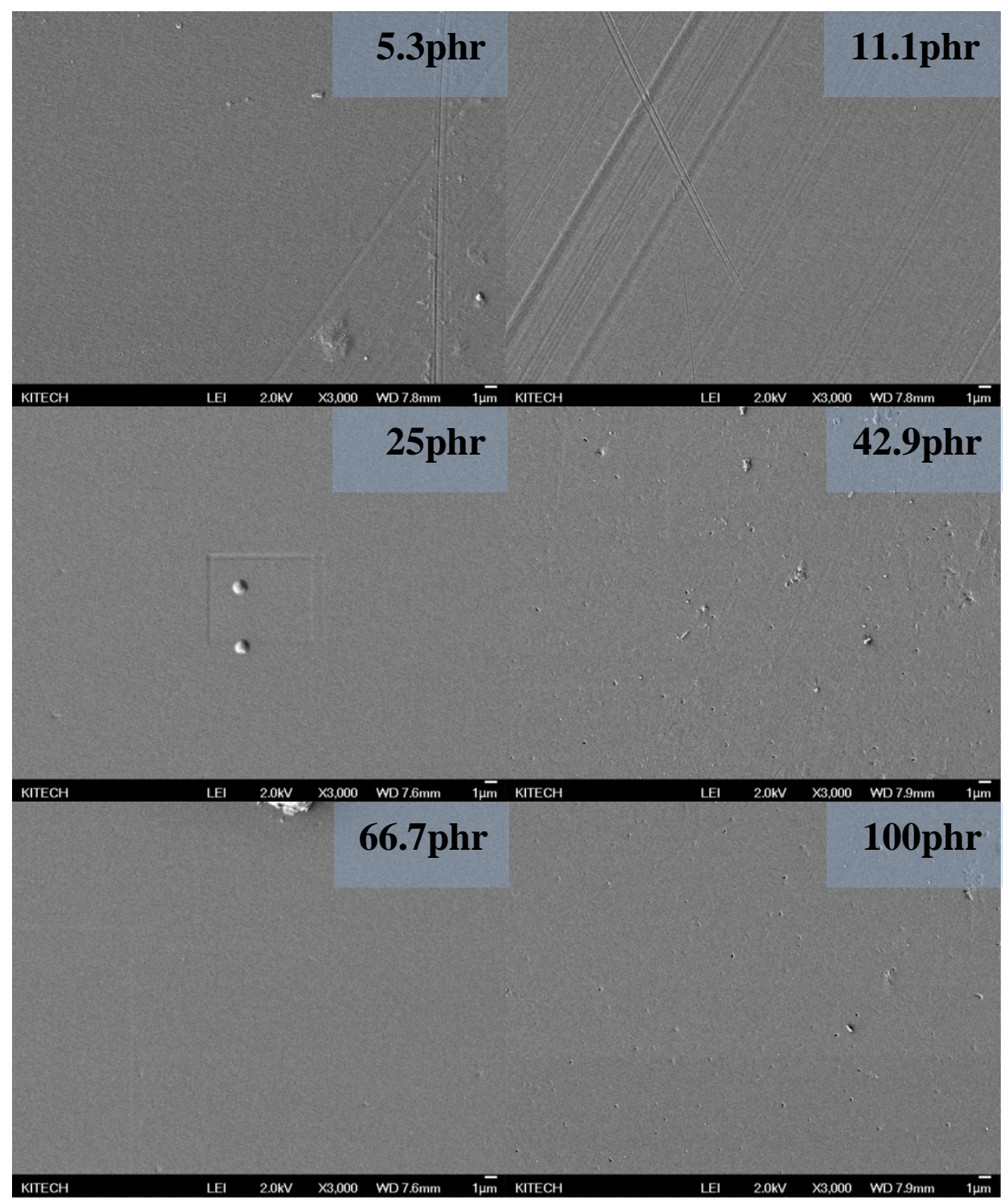

Figure S73. SEM image set of PVC-2,3-DIAF films 


\section{BOND INTERACTION}

\section{Di(2-ethylhexyl) phthalate (DOP)}

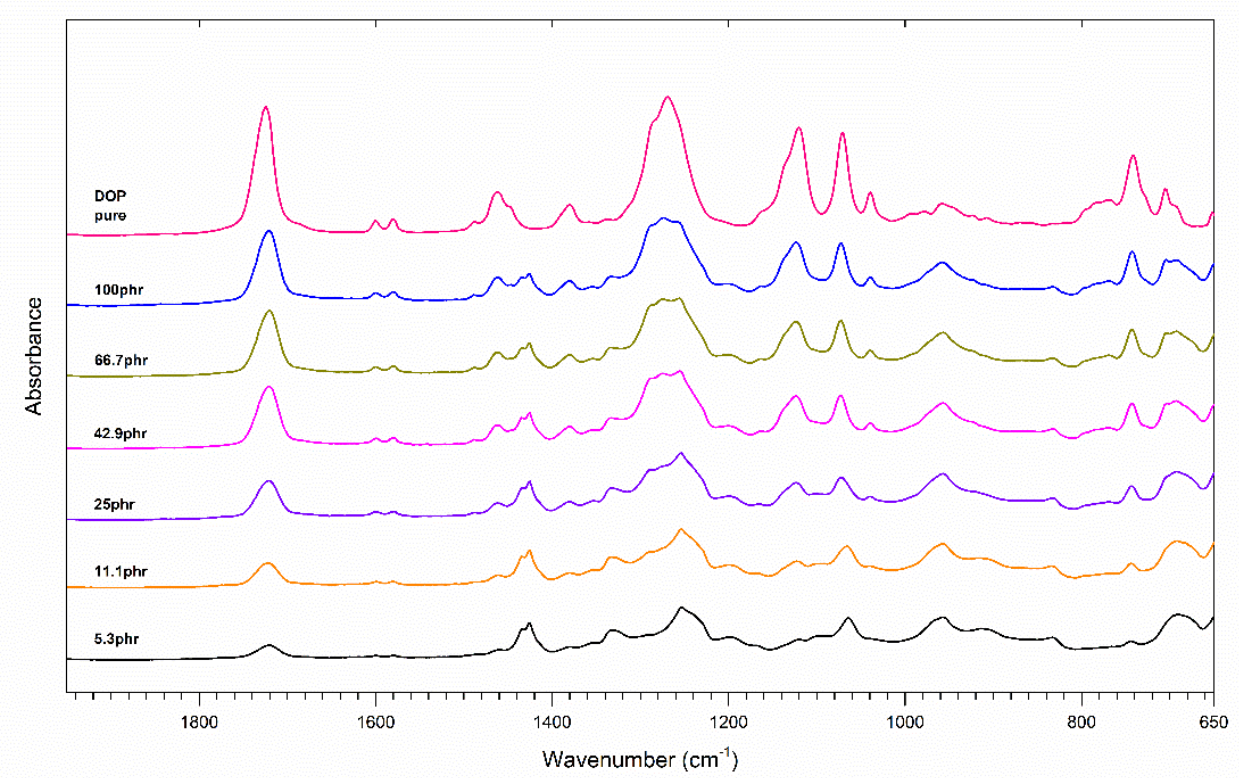

Figure S74. IR absorption bands of pure DOP, and PVC-DOP films

Table S9. Differences in wavenumber of PVC-DOP films compared to pure DOP

\begin{tabular}{l|c|c|c|c|c|c}
\hline \multicolumn{1}{c|}{ Pure DOP } & \multicolumn{5}{|c}{ DOP content (phr) in PVC } \\
\hline $1724 \mathrm{~cm}^{-1}, \mathrm{vC}=\mathrm{O}$ & $-4 \mathrm{~cm}^{-1}$ & $-4 \mathrm{~cm}^{-1}$ & $-4 \mathrm{~cm}^{-1}$ & $-4 \mathrm{~cm}^{-1}$ & $-4 \mathrm{~cm}^{-1}$ & $-4 \mathrm{~cm}^{-1}$ \\
$1600 \mathrm{~cm}^{-1}, \mathrm{vC}=\mathrm{C}$ & $0 \mathrm{~cm}^{-1}$ & $0 \mathrm{~cm}^{-1}$ & $0 \mathrm{~cm}^{-1}$ & $0 \mathrm{~cm}^{-1}$ & $0 \mathrm{~cm}^{-1}$ & $0 \mathrm{~cm}^{-1}$ \\
$1579 \mathrm{~cm}^{-1}, \mathrm{VC}=\mathrm{C}$ & $0 \mathrm{~cm}^{-1}$ & $0 \mathrm{~cm}^{-1}$ & $0 \mathrm{~cm}^{-1}$ & $0 \mathrm{~cm}^{-1}$ & $0 \mathrm{~cm}^{-1}$ & $0 \mathrm{~cm}^{-1}$ \\
$1269 \mathrm{~cm}^{-1}, \mathrm{VC}-\mathrm{O}$ & $-8 \mathrm{~cm}^{-1}$ & $-13 \mathrm{~cm}^{-1}$ & $-14 \mathrm{~cm}^{-1}$ & $-15 \mathrm{~cm}^{-1}$ & $-15 \mathrm{~cm}^{-1}$ & $-16 \mathrm{~cm}^{-1}$ \\
$1178 \mathrm{~cm}^{-1}, \mathrm{VC}-\mathrm{O}$ & $+2 \mathrm{~cm}^{-1}$ & $+2 \mathrm{~cm}^{-1}$ & $+2 \mathrm{~cm}^{-1}$ & $+2 \mathrm{~cm}^{-1}$ & $+1 \mathrm{~cm}^{-1}$ & $+1 \mathrm{~cm}^{-1}$ \\
$1064 \mathrm{~cm}^{-1}$ & $+1 \mathrm{~cm}^{-1}$ & $+1 \mathrm{~cm}^{-1}$ & $+1 \mathrm{~cm}^{-1}$ & $0 \mathrm{~cm}^{-1}$ & $-3 \mathrm{~cm}^{-1}$ & $-4 \mathrm{~cm}^{-1}$ \\
$741 \mathrm{~cm}^{-1}$ & $+2 \mathrm{~cm}^{-1}$ & $+2 \mathrm{~cm}^{-1}$ & $+2 \mathrm{~cm}^{-1}$ & $+2 \mathrm{~cm}^{-1}$ & $+2 \mathrm{~cm}^{-1}$ & $+2 \mathrm{~cm}^{-1}$ \\
$690 \mathrm{~cm}^{-1}, \mathrm{C}-\mathrm{Cl}$ of PVC & $+1 \mathrm{~cm}^{-1}$ & $+1 \mathrm{~cm}^{-1}$ & $+1 \mathrm{~cm}^{-1}$ & $+1 \mathrm{~cm}^{-1}$ & $+1 \mathrm{~cm}^{-1}$ & $+1 \mathrm{~cm}^{-1}$ \\
\hline
\end{tabular}




\section{Di-n-butyl furan-2,5-dicarboxylate (2,5-DBF)}

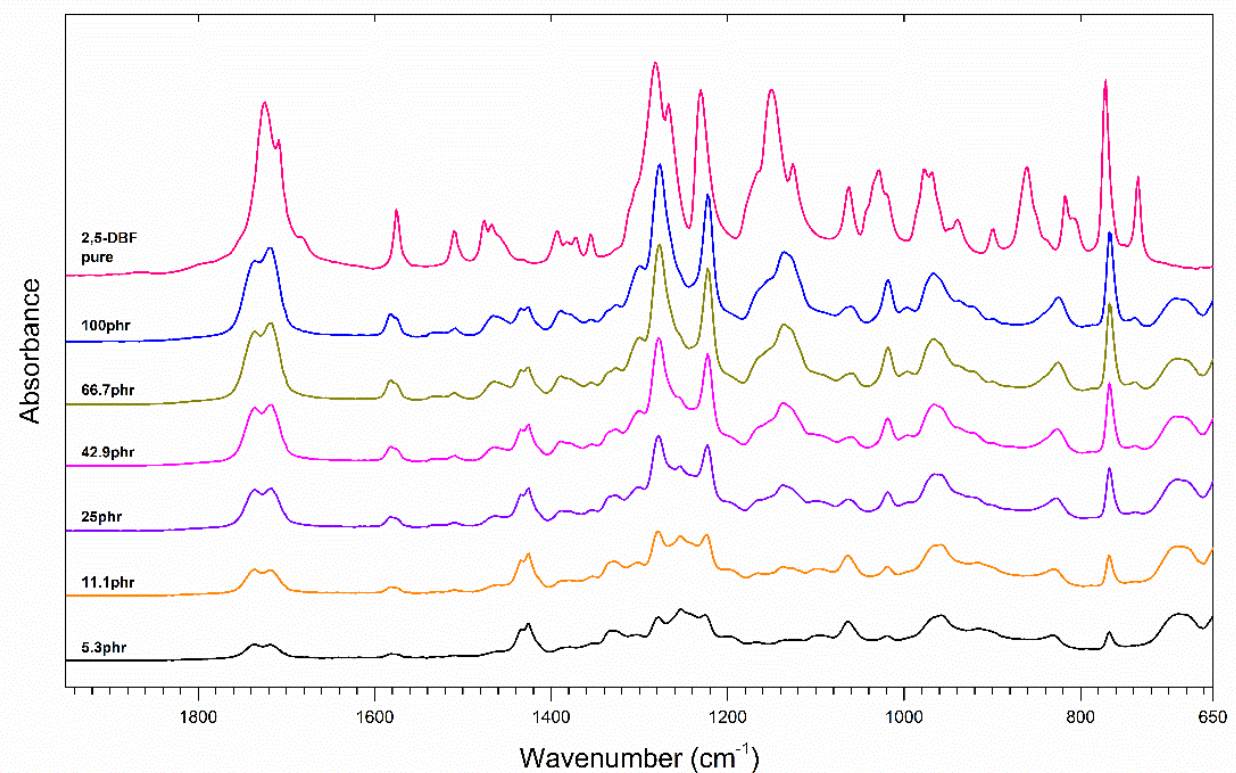

Figure S75. IR absorption bands of pure 2,5-DBF, and PVC-2,5-DBF films

Table S10. Differences in wavenumber of PVC-2,5-DBF films compared to pure 2,5-DBF

\begin{tabular}{l|c|c|c|c|c|c}
\hline \multicolumn{1}{c|}{ Pure 2,5-DBF } & \multicolumn{5}{|c}{$2,5-\mathrm{DBF}$ content (phr) in PVC } \\
\hline $1724 \mathrm{~cm}^{-1}, v \mathrm{C}=\mathrm{O}$ & 100 & 66.7 & 42.9 & 25.0 & 11.1 & 5.3 \\
\hline $1709 \mathrm{~cm}^{-1}, v \mathrm{C}=\mathrm{O}$ & $+12 \mathrm{~cm}^{-1}$ & $+12 \mathrm{~cm}^{-1}$ & $+12 \mathrm{~cm}^{-1}$ & $+12 \mathrm{~cm}^{-1}$ & $+12 \mathrm{~cm}^{-1}$ & $+12 \mathrm{~cm}^{-1}$ \\
$1576 \mathrm{~cm}^{-1}, v \mathrm{C}=\mathrm{C}$ & $+8 \mathrm{~cm}^{-1}$ & $+8 \mathrm{~cm}^{-1}$ & $+8 \mathrm{~cm}^{-1}$ & $+8 \mathrm{~cm}^{-1}$ & $+8 \mathrm{~cm}^{-1}$ & $+8 \mathrm{~cm}^{-1}$ \\
$1510 \mathrm{~cm}^{-1}, v \mathrm{C}=\mathrm{C}$ & $0 \mathrm{~cm}^{-1}$ & $+6 \mathrm{~cm}^{-1}$ & $+6 \mathrm{~cm}^{-1}$ & $+6 \mathrm{~cm}^{-1}$ & $+6 \mathrm{~cm}^{-1}$ & $+6 \mathrm{~cm}^{-1}$ \\
$1282 \mathrm{~cm}^{-1}, v \mathrm{C}-\mathrm{O}$ & $-4 \mathrm{~cm}^{-1}$ & $-4 \mathrm{~cm}^{-1}$ & $-4 \mathrm{~cm}^{-1}$ & $-3 \mathrm{~cm}^{-1}$ & $-3 \mathrm{~cm}^{-1}$ & $-3 \mathrm{~cm}^{-1}$ \\
$1230 \mathrm{~cm}^{-1}, v \mathrm{C}-\mathrm{O}$ & $-7 \mathrm{~cm}^{-1}$ & $-7 \mathrm{~cm}^{-1}$ & $-7 \mathrm{~cm}^{-1}$ & $-7 \mathrm{~cm}^{-1}$ & $-6 \mathrm{~cm}^{-1}$ & $-6 \mathrm{~cm}^{-1}$ \\
$1151 \mathrm{~cm}^{-1}, v \mathrm{C}-\mathrm{O}$ & $-14 \mathrm{~cm}^{-1}$ & $-14 \mathrm{~cm}^{-1}$ & $-14 \mathrm{~cm}^{-1}$ & $-14 \mathrm{~cm}^{-1}$ & $-14 \mathrm{~cm}^{-1}$ & $-14 \mathrm{~cm}^{-1}$ \\
$771 \mathrm{~cm}^{-1}$, cis oop & $-3 \mathrm{~cm}^{-1}$ & $-3 \mathrm{~cm}^{-1}$ & $-3 \mathrm{~cm}^{-1}$ & $-3 \mathrm{~cm}^{-1}$ & $-3 \mathrm{~cm}^{-1}$ & $-3 \mathrm{~cm}^{-1}$ \\
\hline $690 \mathrm{~cm}^{-1}, v \mathrm{C}-\mathrm{Cl}$ of PVC & $0 \mathrm{~cm}^{-1}$ & $0 \mathrm{~cm}^{-1}$ & $0 \mathrm{~cm}^{-1}$ & $0 \mathrm{~cm}^{-1}$ & $0 \mathrm{~cm}^{-1}$ & $0 \mathrm{~cm}^{-1}$ \\
\hline
\end{tabular}




\section{Diisoamyl furan-2,5-dicarboxylate (2,5-DIAF)}

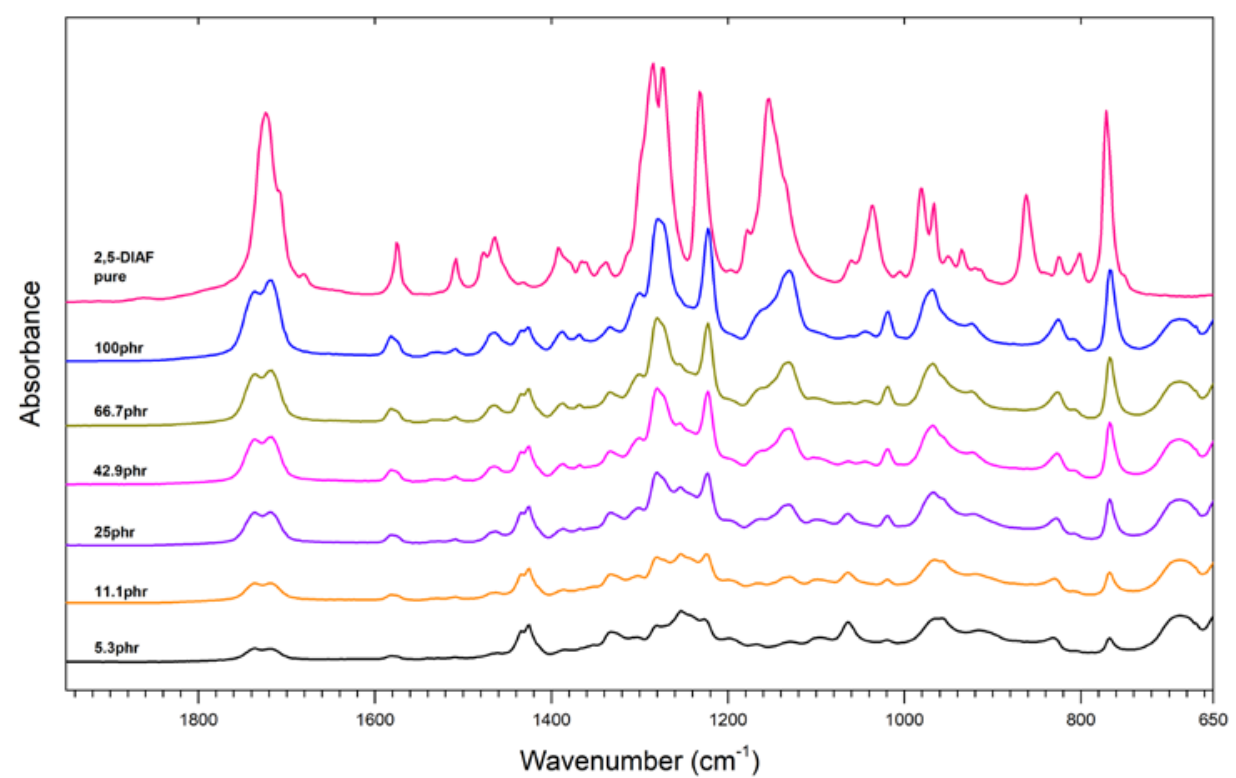

Figure S76. IR absorption bands of pure 2,5-DIAF, and PVC-2,5-DIAF films

Table S11. Differences in wavenumber of PVC-2,5-DIAF films compared to pure 2,5-DIAF

\begin{tabular}{|c|c|c|c|c|c|c|}
\hline \multirow[t]{2}{*}{ Pure 2,5-DIAF } & \multicolumn{6}{|c|}{ 2,5-DIAF content (phr) in PVC } \\
\hline & 100 & 66.7 & 42.9 & 25.0 & 11.1 & 5.3 \\
\hline $1724 \mathrm{~cm}^{-1}, \mathrm{vC}=\mathrm{O}$ & $+12 \mathrm{~cm}^{-1}$ & $+12 \mathrm{~cm}^{-1}$ & $+12 \mathrm{~cm}^{-1}$ & $+12 \mathrm{~cm}^{-1}$ & $+12 \mathrm{~cm}^{-1}$ & $+12 \mathrm{~cm}^{-1}$ \\
\hline $1709 \mathrm{~cm}^{-1}, \mathrm{vC}=\mathrm{O}$ & $+9 \mathrm{~cm}^{-1}$ & $+9 \mathrm{~cm}^{-1}$ & $+9 \mathrm{~cm}^{-1}$ & $+9 \mathrm{~cm}^{-1}$ & $+9 \mathrm{~cm}^{-1}$ & $+9 \mathrm{~cm}^{-1}$ \\
\hline $1576 \mathrm{~cm}^{-1}, v \mathrm{C}=\mathrm{C}$ & $+6 \mathrm{~cm}^{-1}$ & $+6 \mathrm{~cm}^{-1}$ & $+6 \mathrm{~cm}^{-1}$ & $+6 \mathrm{~cm}^{-1}$ & $+6 \mathrm{~cm}^{-1}$ & $+6 \mathrm{~cm}^{-1}$ \\
\hline $1508 \mathrm{~cm}^{-1}, v \mathrm{C}=\mathrm{C}$ & $0 \mathrm{~cm}^{-1}$ & $0 \mathrm{~cm}^{-1}$ & $0 \mathrm{~cm}^{-1}$ & $0 \mathrm{~cm}^{-1}$ & $0 \mathrm{~cm}^{-1}$ & $0 \mathrm{~cm}^{-1}$ \\
\hline $1284 \mathrm{~cm}^{-1}, v \mathrm{C}-\mathrm{O}$ & $-4 \mathrm{~cm}^{-1}$ & $-4 \mathrm{~cm}^{-1}$ & $-4 \mathrm{~cm}^{-1}$ & $-4 \mathrm{~cm}^{-1}$ & $-4 \mathrm{~cm}^{-1}$ & $-4 \mathrm{~cm}^{-1}$ \\
\hline $1232 \mathrm{~cm}^{-1}, \mathrm{vC}-\mathrm{O}$ & $-8 \mathrm{~cm}^{-1}$ & $-8 \mathrm{~cm}^{-1}$ & $-8 \mathrm{~cm}^{-1}$ & $-8 \mathrm{~cm}^{-1}$ & $-8 \mathrm{~cm}^{-1}$ & $-8 \mathrm{~cm}^{-1}$ \\
\hline $1153 \mathrm{~cm}^{-1}, v \mathrm{C}-\mathrm{O}$ & $-23 \mathrm{~cm}^{-1}$ & $-23 \mathrm{~cm}^{-1}$ & $-23 \mathrm{~cm}^{-1}$ & $-23 \mathrm{~cm}^{-1}$ & $-23 \mathrm{~cm}^{-1}$ & $-23 \mathrm{~cm}^{-1}$ \\
\hline $771 \mathrm{~cm}^{-1}$, cis oop & $-3 \mathrm{~cm}^{-1}$ & $-2 \mathrm{~cm}^{-1}$ & $-3 \mathrm{~cm}^{-1}$ & $-3 \mathrm{~cm}^{-1}$ & $-3 \mathrm{~cm}^{-1}$ & $-3 \mathrm{~cm}^{-1}$ \\
\hline $690 \mathrm{~cm}^{-1}, v \mathrm{C}-\mathrm{Cl}$ of PVC & $0 \mathrm{~cm}^{-1}$ & $0 \mathrm{~cm}^{-1}$ & $0 \mathrm{~cm}^{-1}$ & $0 \mathrm{~cm}^{-1}$ & $0 \mathrm{~cm}^{-1}$ & $0 \mathrm{~cm}^{-1}$ \\
\hline
\end{tabular}




\section{Di-n-butyl furan-2,3-dicarboxylate (2,3-DBF)}

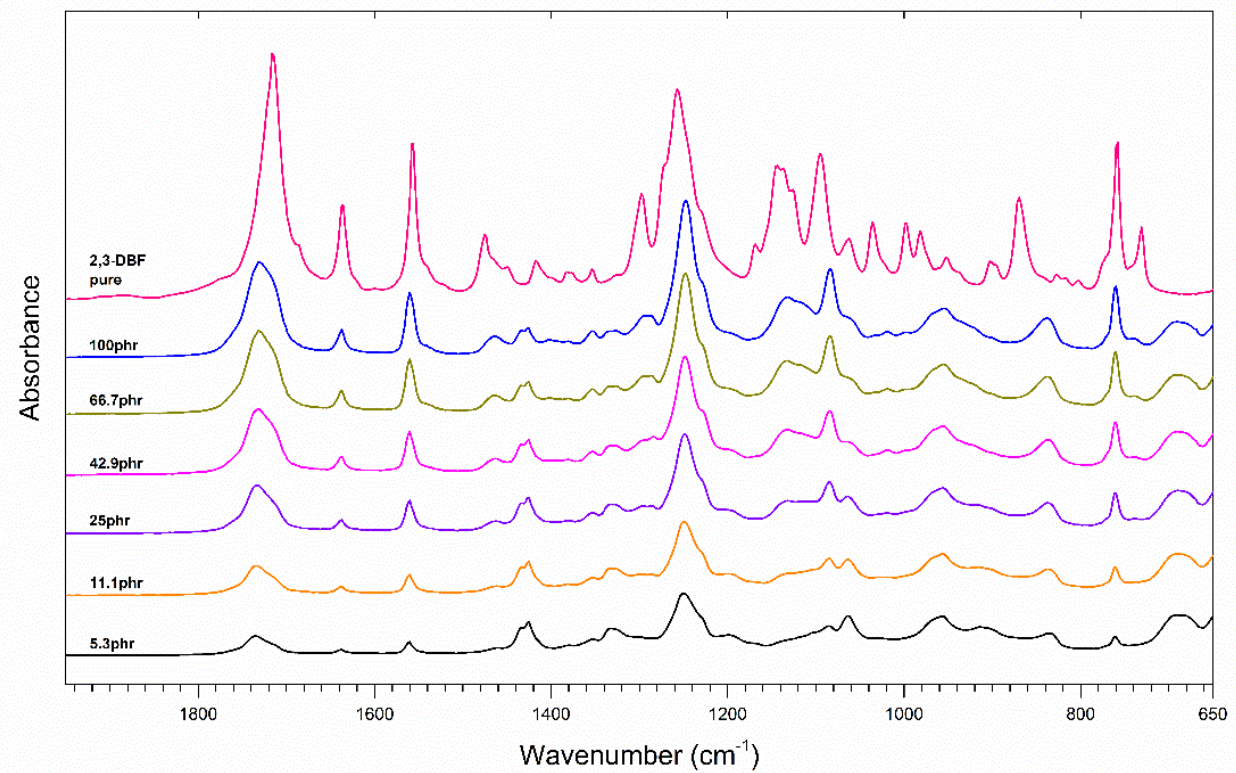

Figure S77. IR absorption bands of pure 2,3-DBF, and PVC-2,3-DBF films

Table S12. Differences in wavenumber of PVC-2,3-DBF films compared to pure 2,3-DBF

\begin{tabular}{|c|c|c|c|c|c|c|}
\hline \multirow[t]{2}{*}{ Pure 2,3-DBF } & \multicolumn{6}{|c|}{ 2,3-DBF content (phr) in PVC } \\
\hline & 100 & 66.7 & 42.9 & 25.0 & 11.1 & 5.3 \\
\hline $1716 \mathrm{~cm}^{-1}, v \mathrm{C}=\mathrm{O}$ & $+14 \mathrm{~cm}^{-1}$ & $+14 \mathrm{~cm}^{-1}$ & $+15 \mathrm{~cm}^{-1}$ & $+17 \mathrm{~cm}^{-1}$ & $+18 \mathrm{~cm}^{-1}$ & $+18 \mathrm{~cm}^{-1}$ \\
\hline $1637 \mathrm{~cm}^{-1}$ & $+1 \mathrm{~cm}^{-1}$ & $+1 \mathrm{~cm}^{-1}$ & $+1 \mathrm{~cm}^{-1}$ & $+1 \mathrm{~cm}^{-1}$ & $+1 \mathrm{~cm}^{-1}$ & $+1 \mathrm{~cm}^{-1}$ \\
\hline $1556 \mathrm{~cm}^{-1}$ & $+5 \mathrm{~cm}^{-1}$ & $+5 \mathrm{~cm}^{-1}$ & $+5 \mathrm{~cm}^{-1}$ & $+5 \mathrm{~cm}^{-1}$ & $+5 \mathrm{~cm}^{-1}$ & $+5 \mathrm{~cm}^{-1}$ \\
\hline $1298 \mathrm{~cm}^{-1}, v \mathrm{C}-\mathrm{O}$ & $-2 \mathrm{~cm}^{-1}$ & $-2 \mathrm{~cm}^{-1}$ & $-2 \mathrm{~cm}^{-1}$ & $-2 \mathrm{~cm}^{-1}$ & - & - \\
\hline $1257 \mathrm{~cm}^{-1}, v \mathrm{C}-\mathrm{O}$ & $0 \mathrm{~cm}^{-1}$ & $0 \mathrm{~cm}^{-1}$ & $0 \mathrm{~cm}^{-1}$ & $0 \mathrm{~cm}^{-1}$ & $0 \mathrm{~cm}^{-1}$ & $0 \mathrm{~cm}^{-1}$ \\
\hline $1144 \mathrm{~cm}^{-1}, v \mathrm{C}-\mathrm{O}$ & $-2 \mathrm{~cm}^{-1}$ & $-2 \mathrm{~cm}^{-1}$ & $-2 \mathrm{~cm}^{-1}$ & $-2 \mathrm{~cm}^{-1}$ & $-1 \mathrm{~cm}^{-1}$ & - \\
\hline $1095 \mathrm{~cm}^{-1}, v \mathrm{C}-\mathrm{O}$ & $-11 \mathrm{~cm}^{-1}$ & $-11 \mathrm{~cm}^{-1}$ & $-11 \mathrm{~cm}^{-1}$ & $-10 \mathrm{~cm}^{-1}$ & $-10 \mathrm{~cm}^{-1}$ & $-10 \mathrm{~cm}^{-1}$ \\
\hline $758 \mathrm{~cm}^{-1}$, cis oop & $+1 \mathrm{~cm}^{-1}$ & $+1 \mathrm{~cm}^{-1}$ & $+1 \mathrm{~cm}^{-1}$ & $+1 \mathrm{~cm}^{-1}$ & $+1 \mathrm{~cm}^{-1}$ & $+1 \mathrm{~cm}^{-1}$ \\
\hline $690 \mathrm{~cm}^{-1}, v \mathrm{C}-\mathrm{Cl}$ of PVC & $0 \mathrm{~cm}^{-1}$ & $0 \mathrm{~cm}^{-1}$ & $0 \mathrm{~cm}^{-1}$ & $0 \mathrm{~cm}^{-1}$ & $0 \mathrm{~cm}^{-1}$ & $0 \mathrm{~cm}^{-1}$ \\
\hline
\end{tabular}




\section{Diisoamyl furan-2,3-dicarboxylate (2,3-DIAF)}

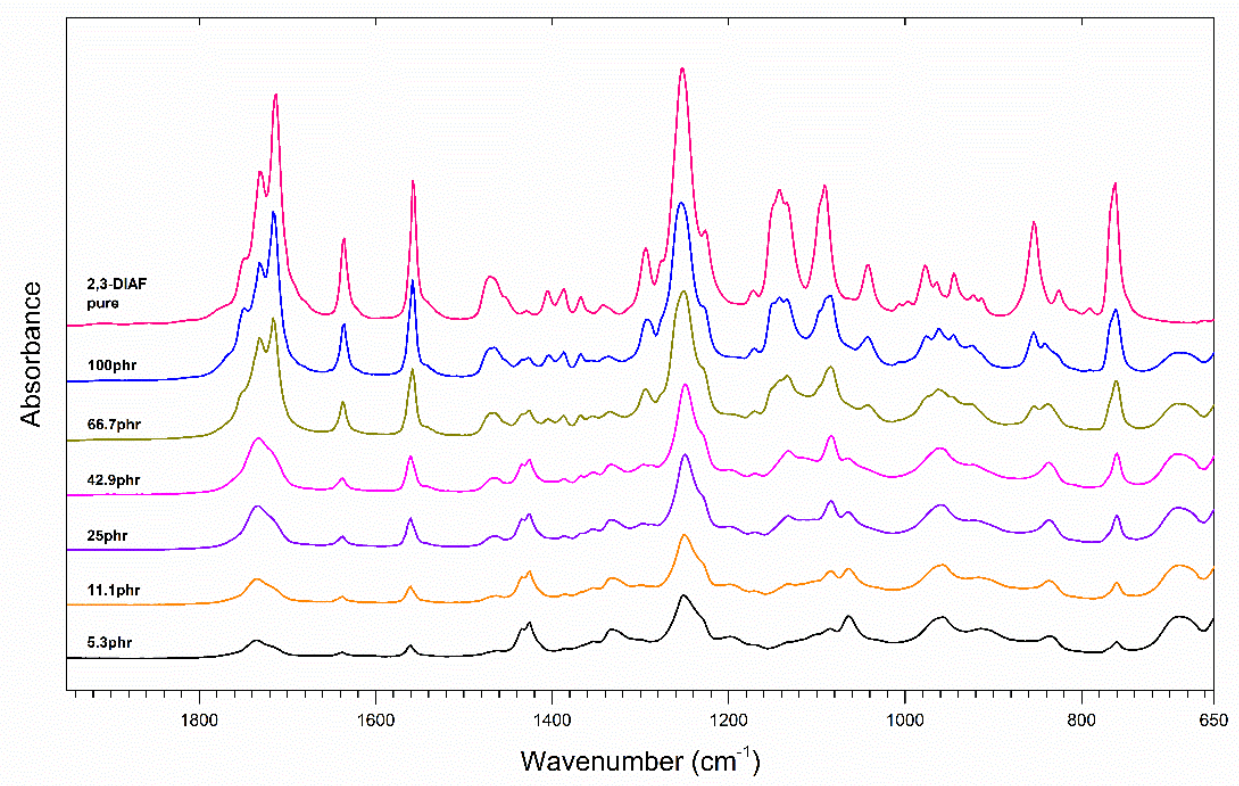

Figure S78. IR absorption bands of pure 2,3-DIAF, and PVC-2,3-DIAF films

Table S13. Differences in wavenumber of PVC-2,3-DIAF films compared to pure 2,3-DIAF

\begin{tabular}{l|c|c|c|c|c|c}
\hline \multicolumn{1}{c|}{ Pure 2,3-DIAF } & \multicolumn{5}{|c}{2,3 -DIAF content (phr) in PVC } \\
\hline $1712 \mathrm{~cm}^{-1}, \mathrm{vC}=\mathrm{O}$ & $+10 \mathrm{~cm}^{-1}$ & $+10 \mathrm{~cm}^{-1}$ & $+18 \mathrm{~cm}^{-1}$ & $+19 \mathrm{~cm}^{-1}$ & $+20 \mathrm{~cm}^{-1}$ & $+21 \mathrm{~cm}^{-1}$ \\
$1635 \mathrm{~cm}^{-1}$ & $+3 \mathrm{~cm}^{-1}$ & $+3 \mathrm{~cm}^{-1}$ & $+3 \mathrm{~cm}^{-1}$ & $+3 \mathrm{~cm}^{-1}$ & $+3 \mathrm{~cm}^{-1}$ & $+3 \mathrm{~cm}^{-1}$ \\
$1558 \mathrm{~cm}^{-1}$ & $+1 \mathrm{~cm}^{-1}$ & $+1 \mathrm{~cm}^{-1}$ & $+3 \mathrm{~cm}^{-1}$ & $+3 \mathrm{~cm}^{-1}$ & $+3 \mathrm{~cm}^{-1}$ & $+4 \mathrm{~cm}^{-1}$ \\
$1294 \mathrm{~cm}^{-1}, \mathrm{VC}-\mathrm{O}$ & $0 \mathrm{~cm}^{-1}$ & $0 \mathrm{~cm}^{-1}$ & $0 \mathrm{~cm}^{-1}$ & - & - & - \\
$1253 \mathrm{~cm}^{-1}, \mathrm{VC}-\mathrm{O}$ & $0 \mathrm{~cm}^{-1}$ & $0 \mathrm{~cm}^{-1}$ & $0 \mathrm{~cm}^{-1}$ & $-1 \mathrm{~cm}^{-1}$ & $0 \mathrm{~cm}^{-1}$ & $-1 \mathrm{~cm}^{-1}$ \\
$1142 \mathrm{~cm}^{-1}, \mathrm{VC}-\mathrm{O}$ & $-8 \mathrm{~cm}^{-1}$ & $-9 \mathrm{~cm}^{-1}$ & $-10 \mathrm{~cm}^{-1}$ & $-10 \mathrm{~cm}^{-1}$ & $-11 \mathrm{~cm}^{-1}$ & $-12 \mathrm{~cm}^{-1}$ \\
$1092 \mathrm{~cm}^{-1}, \mathrm{VC}-\mathrm{O}$ & $-6 \mathrm{~cm}^{-1}$ & $-7 \mathrm{~cm}^{-1}$ & $-7 \mathrm{~cm}^{-1}$ & $-7 \mathrm{~cm}^{-1}$ & $-7 \mathrm{~cm}^{-1}$ & $-6 \mathrm{~cm}^{-1}$ \\
$762 \mathrm{~cm}^{-1}$, cis oop & $0 \mathrm{~cm}^{-1}$ & $-1 \mathrm{~cm}^{-1}$ & $-2 \mathrm{~cm}^{-1}$ & $-2 \mathrm{~cm}^{-1}$ & $-2 \mathrm{~cm}^{-1}$ & $-2 \mathrm{~cm}^{-1}$ \\
$690 \mathrm{~cm}^{-1}, \mathrm{C}-\mathrm{Cl}$ of PVC & $0 \mathrm{~cm}^{-1}$ & $0 \mathrm{~cm}^{-1}$ & $0 \mathrm{~cm}^{-1}$ & $0 \mathrm{~cm}^{-1}$ & $0 \mathrm{~cm}^{-1}$ & $0 \mathrm{~cm}^{-1}$ \\
\hline
\end{tabular}




\section{GLASS TRANSITION TEMPERATURE}

Differential scanning calorimetry (DSC) was studied on a DSC 8000 (PerkinElmer, USA). Each sample weight was between 7 and $10 \mathrm{mg}$ (DOP samples weight ranged from 18 to $21 \mathrm{mg}$ ). The running program included six steps (shown below, all under nitrogen gas flow of $20 \mathrm{~mL} / \mathrm{min}$ ):

$$
\begin{aligned}
& 1 \text { - hold temperature at } 25^{\circ} \mathrm{C} \text { for one minute } \\
& 2 \text { - rise to } T_{\text {final }}{ }^{\circ} \mathrm{C} \text { at } 10{ }^{\circ} \mathrm{C} / \mathrm{min} \text { rate (for DOP, } T_{\text {final }} \text { is } 150{ }^{\circ} \mathrm{C} \text {; for all others } T_{\text {final }} \text { is } 200{ }^{\circ} \mathrm{C} \text { ) } \\
& 3 \text { - hold for two minutes } \\
& 4 \text { - cool from } T_{\text {final }}{ }^{\circ} \mathrm{C} \text { to }-50{ }^{\circ} \mathrm{C} \text { at } 50{ }^{\circ} \mathrm{C} / \text { min rate } \\
& 5 \text { - hold temperature at }-50{ }^{\circ} \mathrm{C} \text { for three minutes } \\
& 6 \text { - heat from }-50{ }^{\circ} \mathrm{C} \text { to } T_{\text {final }}{ }^{\circ} \mathrm{C} \text { at } 10^{\circ} \mathrm{C} / \text { min rate }
\end{aligned}
$$

$T_{\mathrm{g}}$ was extrapolated as the half-height midpoint of the change in heat capacity on the thermal curve of the last step (Step 6).

Table S14. Glass transition temperatures of PVC-PLS films

\begin{tabular}{c|ccccc}
\hline $\begin{array}{l}\text { Content } \\
(\mathrm{phr})\end{array}$ & \multicolumn{5}{|c}{ Glass Transition Temperature $\left({ }^{\circ} \mathrm{C}\right)$} \\
\hline 0 & DOP & $2,5-\mathrm{DBF}$ & $2,5-\mathrm{DIAF}$ & $2,3-\mathrm{DBF}$ & 2,3-DIAF \\
\hline 5.3 & 80.77 & 80.77 & 80.77 & 80.77 & 80.77 \\
11.1 & 61.53 & 59.83 & 62.44 & 62.74 & 66.72 \\
25.0 & 49.17 & 51.00 & 53.25 & 56.18 & 58.34 \\
42.9 & 26.08 & 29.20 & 35.13 & 41.11 & 44.65 \\
66.7 & 19.86 & 20.49 & 21.40 & 29.77 & 33.05 \\
100 & 9.52 & 7.67 & 8.46 & 20.38 & 21.65 \\
\hline
\end{tabular}



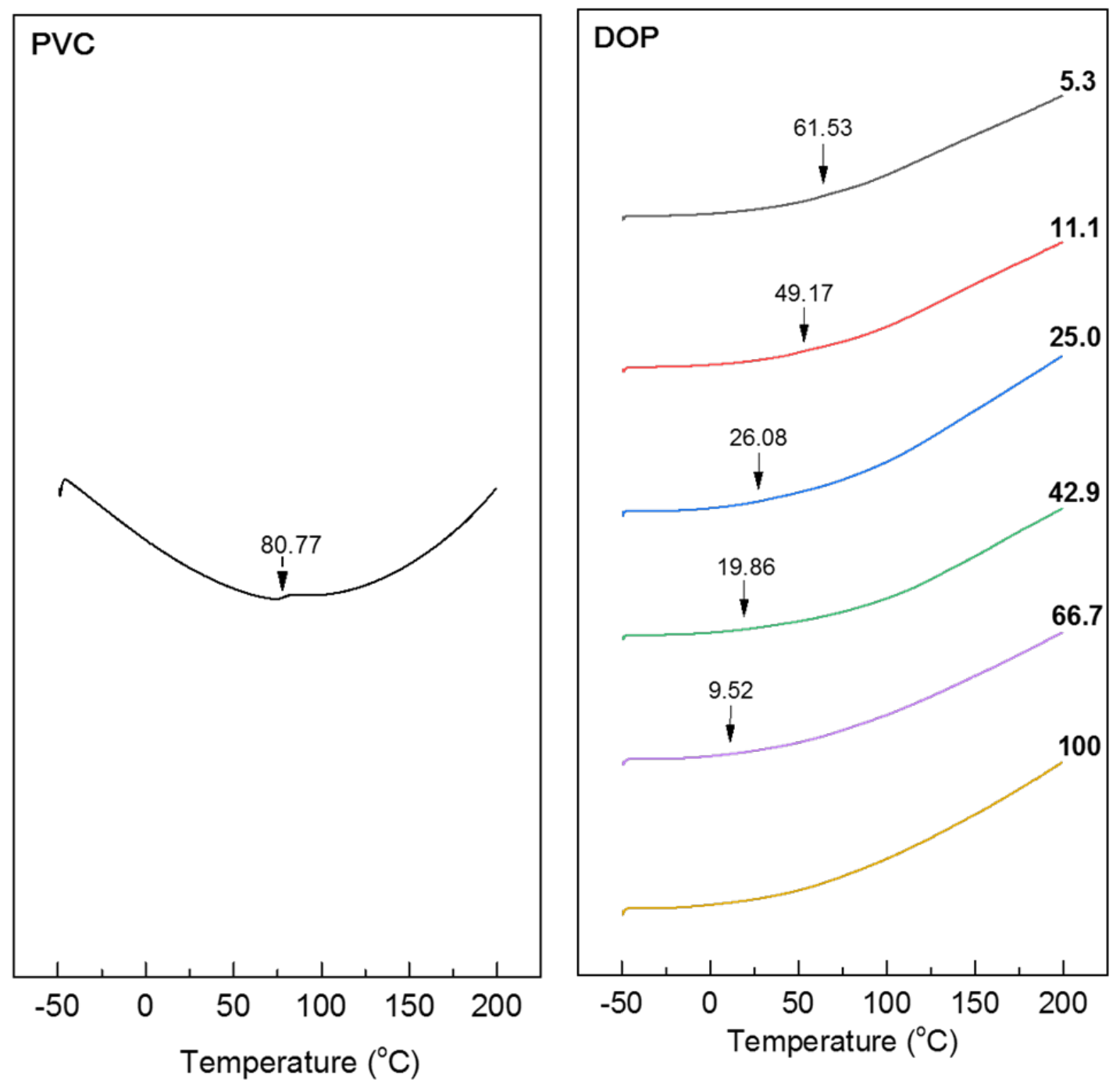

Figure S79. DSC thermograms of PVC and PVC-PLS films 


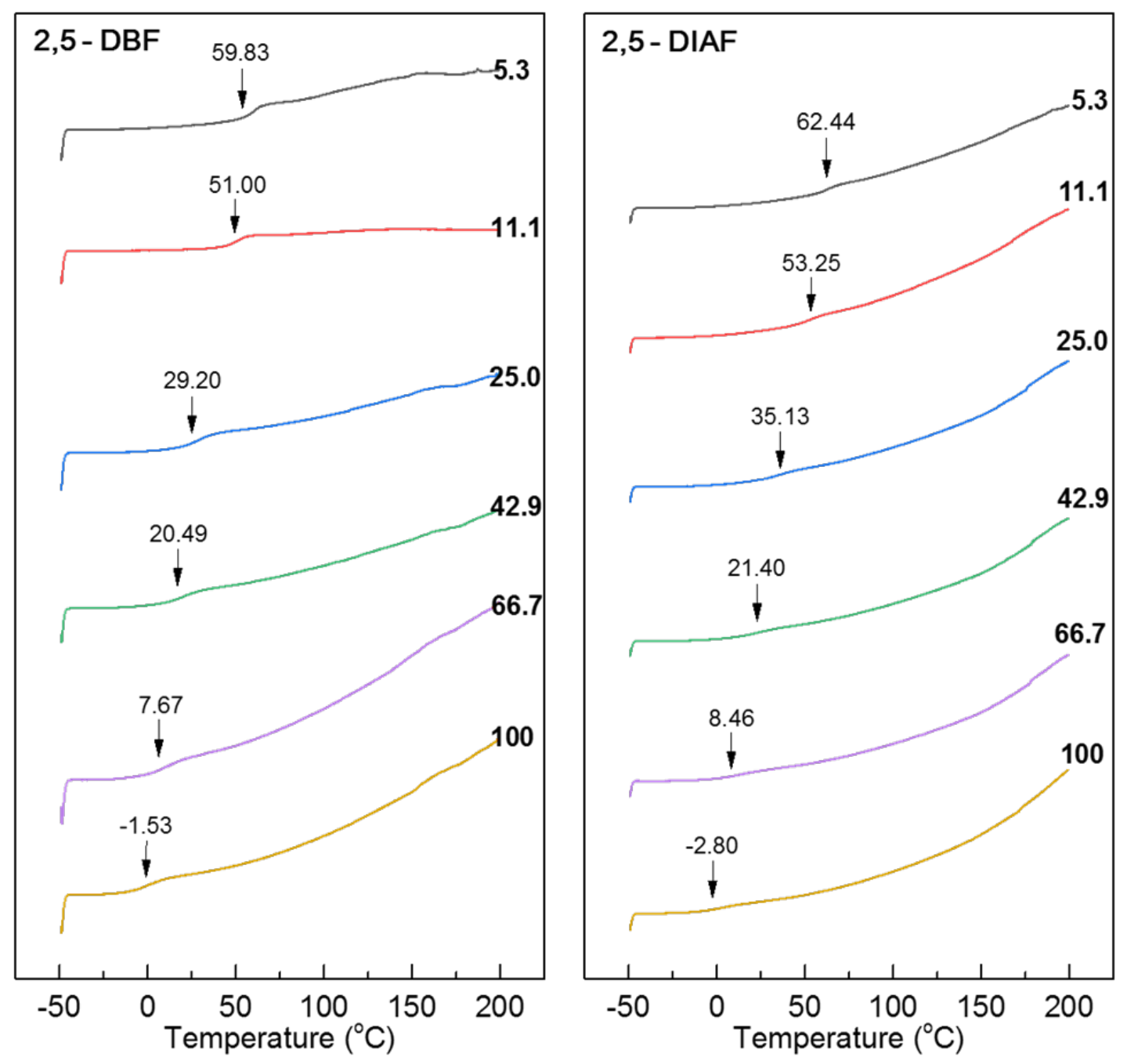

Figure S80. DSC thermograms of PVC and PVC-PLS films (continued) 

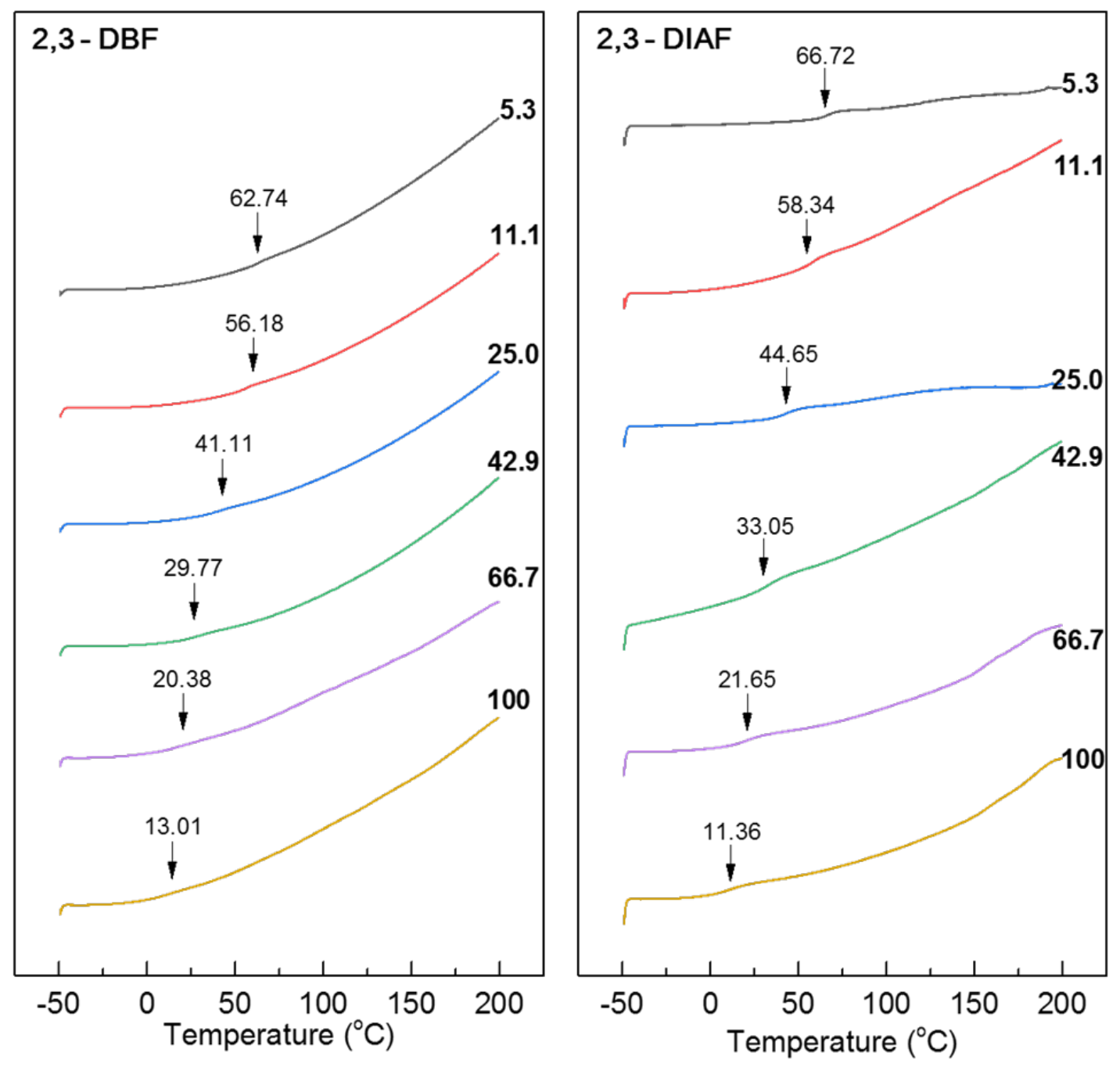

Figure S81. DSC thermograms of PVC and PVC-PLS films (continued) 


\section{TENSILE PROPERTIES}

Tensile properties of polymer-plasticizer materials were tested on a Universal Testing Machine (UTM) from Z005 model (Zwick Roell, Germany), based upon ASTM D882 - 12 standard. At least seven or more tensile specimens were prepared from each film. Specimens were preconditioned at $23 \pm 0.5{ }^{\circ} \mathrm{C}$, and $50 \pm 5 \%$ rh for not less than 48 hours. The standard distance between grips was $25 \mathrm{~mm}$; rate of grip separation was $2.5 \mathrm{~mm} / \mathrm{min}$; initial strain rate was $0.1 \mathrm{~min}^{-}$ 1; pre-load force was $0.1 \mathrm{MPa}$; thickness of specimen was measured by an electronic micrometer, right before the test started. For those curves having Hookean region, E-Modulus is (tangent) slope value of initial linear part of a curve. For those curves without Hookean region, E-Modulus is 0.50\% secant modulus (from the corrected zero-strain point). The standard deviation (estimated) is uniquely provided by the standard, as follows:

$$
s=\sqrt{\frac{\sum X^{2}-n \bar{X}^{2}}{n-1}}
$$

Table S15. Tensile properties of investigated PVC-PLS films

\begin{tabular}{lcccc}
\hline Sample & $n^{*}$ & $\begin{array}{c}\text { Elongation } \\
\text { at break (\%) }\end{array}$ & $\begin{array}{c}\text { Stress at break } \\
(\mathrm{MPa})\end{array}$ & $\begin{array}{c}\text { E-Modulus } \\
(\mathrm{MPa})\end{array}$ \\
\hline DOP 42.9phr & 7 & $166.95 \pm 3.95$ & $19.38 \pm 0.92$ & $314.2 \pm 6.6$ \\
DOP 66.7phr & 13 & $166.71 \pm 5.16$ & $14.78 \pm 0.48$ & $71.7 \pm 6.4$ \\
DOP 100phr & 7 & $169.93 \pm 7.54$ & $11.37 \pm 0.48$ & $28.5 \pm 1.5$ \\
\hline 2,5-DBF 42.9phr & 10 & $165.39 \pm 12.07$ & $17.81 \pm 0.76$ & $268.9 \pm 13.5$ \\
2,5-DBF 66.7phr & 10 & $150.22 \pm 5.61$ & $11.03 \pm 0.48$ & $68.3 \pm 6.5$ \\
2,5-DBF 100phr & 7 & $150.05 \pm 3.60$ & $8.46 \pm 0.20$ & $36.4 \pm 1.4$ \\
\hline
\end{tabular}




\begin{tabular}{lcccc}
\hline 2,5-DIAF 42.9phr & 7 & $185.57 \pm 8.69$ & $16.83 \pm 0.97$ & $222.5 \pm 20.4$ \\
2,5-DIAF 66.7phr & 9 & $165.16 \pm 3.85$ & $12.80 \pm 0.33$ & $93.6 \pm 7.8$ \\
2,5-DIAF 100phr & 7 & $163.86 \pm 4.19$ & $9.06 \pm 0.29$ & $27.6 \pm 1.4$ \\
\hline 2,3-DBF 42.9phr & 7 & $190.08 \pm 5.37$ & $14.68 \pm 0.60$ & $258.7 \pm 22.2$ \\
2,3-DBF 66.7phr & 9 & $169.76 \pm 3.43$ & $11.34 \pm 0.41$ & $77.1 \pm 3.4$ \\
2,3-DBF 100phr & 9 & $171.13 \pm 4.85$ & $9.58 \pm 0.87$ & $42.4 \pm 2.0$ \\
\hline 2,3-DIAF 42.9phr & 7 & $170.97 \pm 10.19$ & $18.22 \pm 0.69$ & $264.4 \pm 18.8$ \\
2,3-DIAF 66.7phr & 9 & $168.00 \pm 6.55$ & $12.60 \pm 0.51$ & $78.3 \pm 7.4$ \\
2,3-DIAF 100phr & 8 & $184.71 \pm 5.75$ & $11.62 \pm 0.32$ & $57.8 \pm 3.2$ \\
\hline
\end{tabular}

* where: $\quad n$ is number of test specimens 


\section{THERMAL DEGRADATION}

A TGA 4000 System (PerkinElmer, USA) was used to performed thermogravimetric analyses (TGA). About 12-15 mg of each film with no heat stabilizer was put into a ceramic cup, without lid. Heating sample from $25^{\circ} \mathrm{C}$ to $700{ }^{\circ} \mathrm{C}$ at $10{ }^{\circ} \mathrm{C} / \mathrm{min}$ under nitrogen gas flow (20 mL/min).

\section{Di-n-butyl furan-2,5-dicarboxylate (2,5-DBF)}

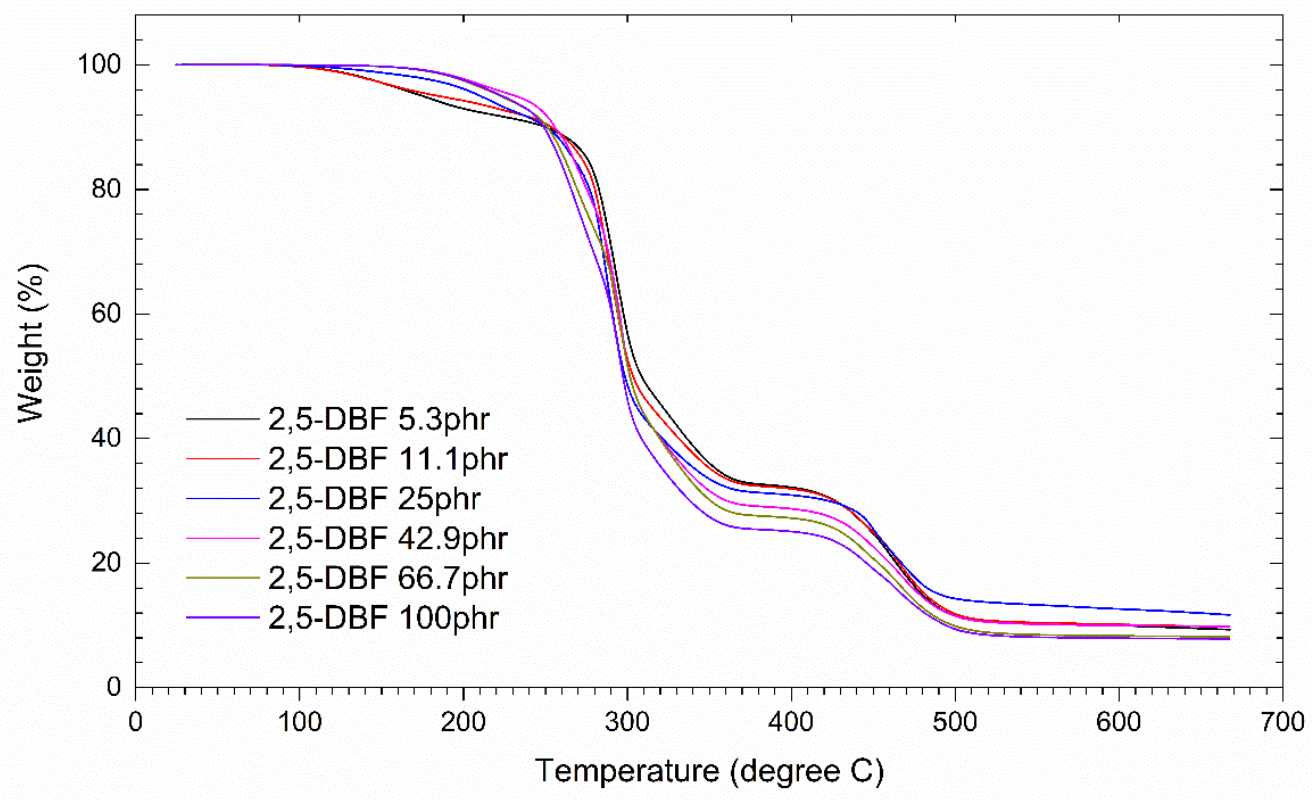

Figure S80. Thermal degradation curves of PVC-2,5-DBF films

Table S16. Thermal degradation data of PVC-2,5-DBF films

\begin{tabular}{lcccccc}
\hline Film & $\mathrm{T}_{5 \%}$ & $\mathrm{~T}_{10 \%}$ & $\mathrm{~T}_{25 \%}$ & $\mathrm{~T}_{50 \%}$ & $\mathrm{~T}_{75 \%}$ & \% Residue 650 ${ }^{\circ} \mathrm{C}$ \\
\hline 2,5-DBF 5.3phr & 175.18 & 250.14 & 286.96 & 308.56 & 449.08 & 9.503 \\
2,5-DBF 11.1phr & 184.94 & 253.74 & 284.19 & 303.44 & 449.84 & 9.871 \\
2,5-DBF 25phr & 210.45 & 250.61 & 281.83 & 298.47 & 451.32 & 11.979 \\
2,5-DBF 42.9phr & 231.74 & 255.77 & 283.06 & 301.53 & 439.85 & 9.871 \\
2,5-DBF 66.7phr & 222.70 & 251.28 & 277.47 & 301.53 & 430.18 & 8.216 \\
2,5-DBF 100phr & 223.68 & 249.05 & 272.40 & 297.23 & 402.60 & 7.866 \\
\hline
\end{tabular}




\section{Diisoamyl furan-2,5-dicarboxylate (2,5-DIAF)}

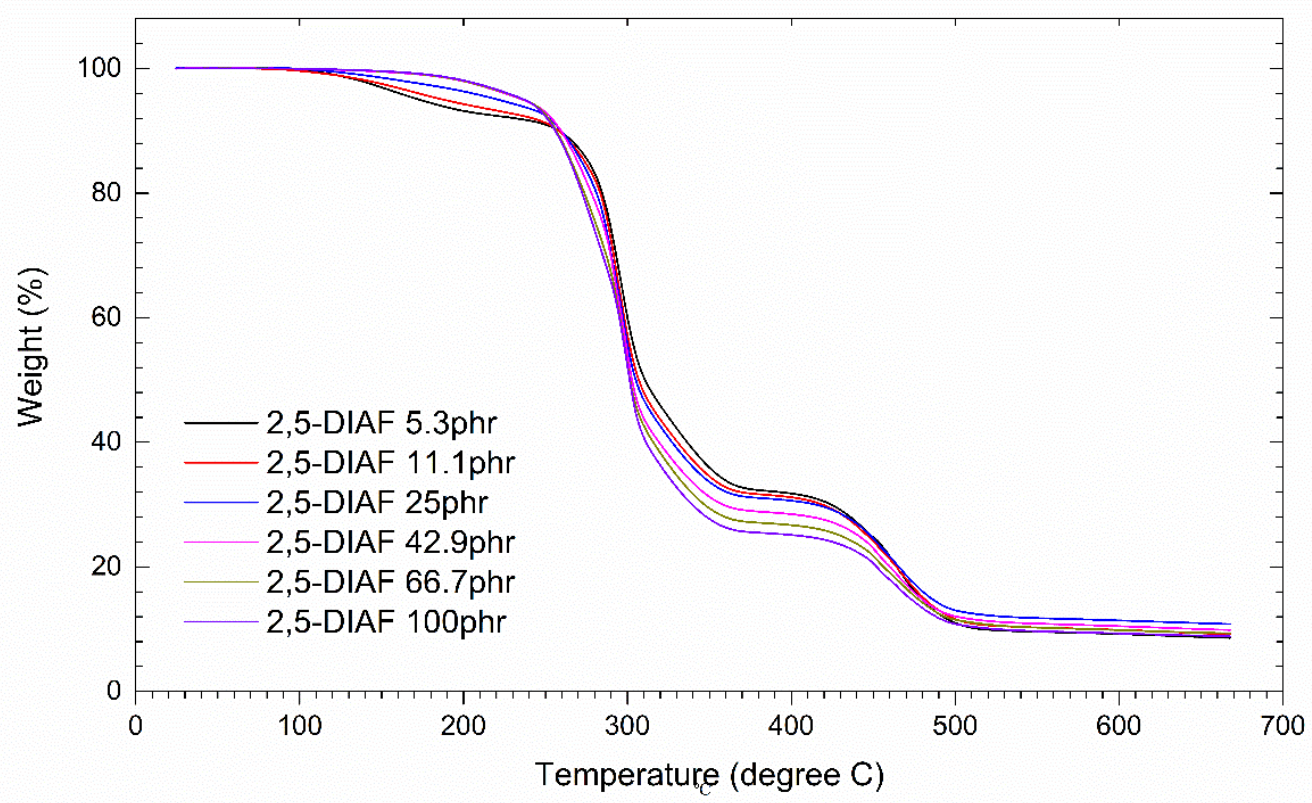

Figure S82. Thermal degradation curves of PVC-2,5-DIAF films

Table S17. Thermal degradation data of PVC-2,5-DIAF films

\begin{tabular}{lcccccc}
\hline Film & $\mathrm{T}_{5 \%}$ & $\mathrm{~T}_{10 \%}$ & $\mathrm{~T}_{25 \%}$ & $\mathrm{~T}_{50 \%}$ & $\mathrm{~T}_{75 \%}$ & \% Residue $650{ }^{\circ} \mathrm{C}$ \\
\hline 2,5-DIAF 5.3phr & 173.04 & 258.16 & 289.40 & 310.76 & 448.85 & 8.757 \\
2,5-DIAF 11.1phr & 187.97 & 257.99 & 288.16 & 306.94 & 446.51 & 9.365 \\
2,5-DIAF 25phr & 221.15 & 259.69 & 286.51 & 305.08 & 448.43 & 10.963 \\
2,5-DIAF 42.9phr & 236.06 & 259.42 & 285.14 & 302.82 & 441.03 & 10.010 \\
2,5-DIAF 66.7phr & 236.98 & 256.69 & 280.79 & 301.58 & 429.93 & 9.448 \\
2,5-DIAF 100phr & 237.15 & 255.91 & 278.79 & 301.33 & 404.77 & 8.970 \\
\hline
\end{tabular}




\section{Di-n-butyl furan-2,3-dicarboxylate (2,3-DBF)}

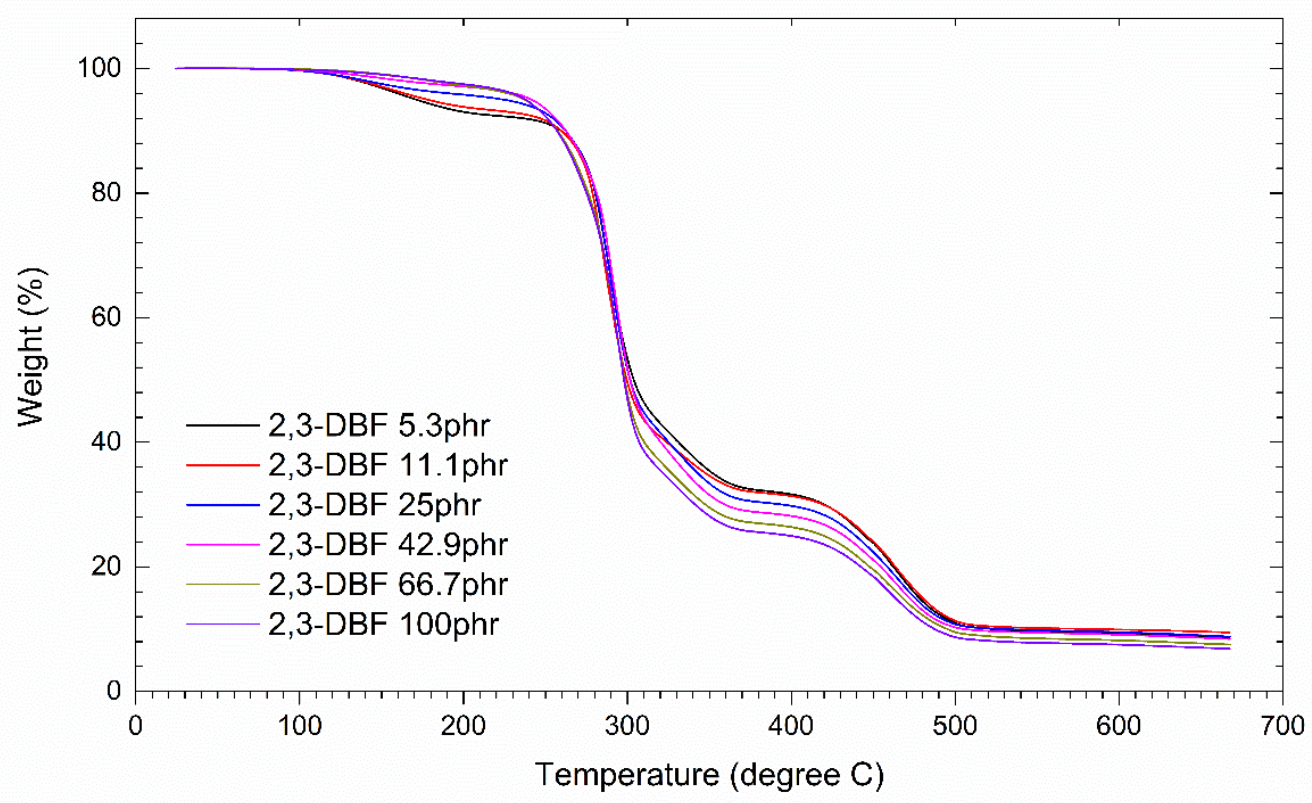

Figure S83. Thermal degradation curves of PVC-2,3-DBF films

Table S18. Thermal degradation data of PVC-2,3-DBF films

\begin{tabular}{lcccccc}
\hline Film & $\mathrm{T}_{5 \%}$ & $\mathrm{~T}_{10 \%}$ & $\mathrm{~T}_{25 \%}$ & $\mathrm{~T}_{50 \%}$ & $\mathrm{~T}_{75 \%}$ & \% Residue $650{ }^{\circ} \mathrm{C}$ \\
\hline 2,3-DBF 5.3phr & 171.27 & 259.69 & 284.15 & 303.90 & 444.68 & 8.721 \\
2,3-DBF 11.1phr & 178.19 & 259.80 & 282.22 & 299.60 & 445.89 & 9.623 \\
2,3-DBF 25phr & 224.53 & 262.27 & 284.33 & 301.66 & 439.21 & 9.013 \\
2,3-DBF 42.9phr & 241.61 & 262.54 & 285.72 & 301.62 & 432.28 & 8.628 \\
2,3-DBF 66.7phr & 237.34 & 257.35 & 281.91 & 298.39 & 419.78 & 7.715 \\
2,3-DBF 100phr & 237.92 & 256.93 & 281.25 & 297.93 & 399.06 & 7.033 \\
\hline
\end{tabular}




\section{Diisoamyl furan-2,3-dicarboxylate (2,3-DIAF)}

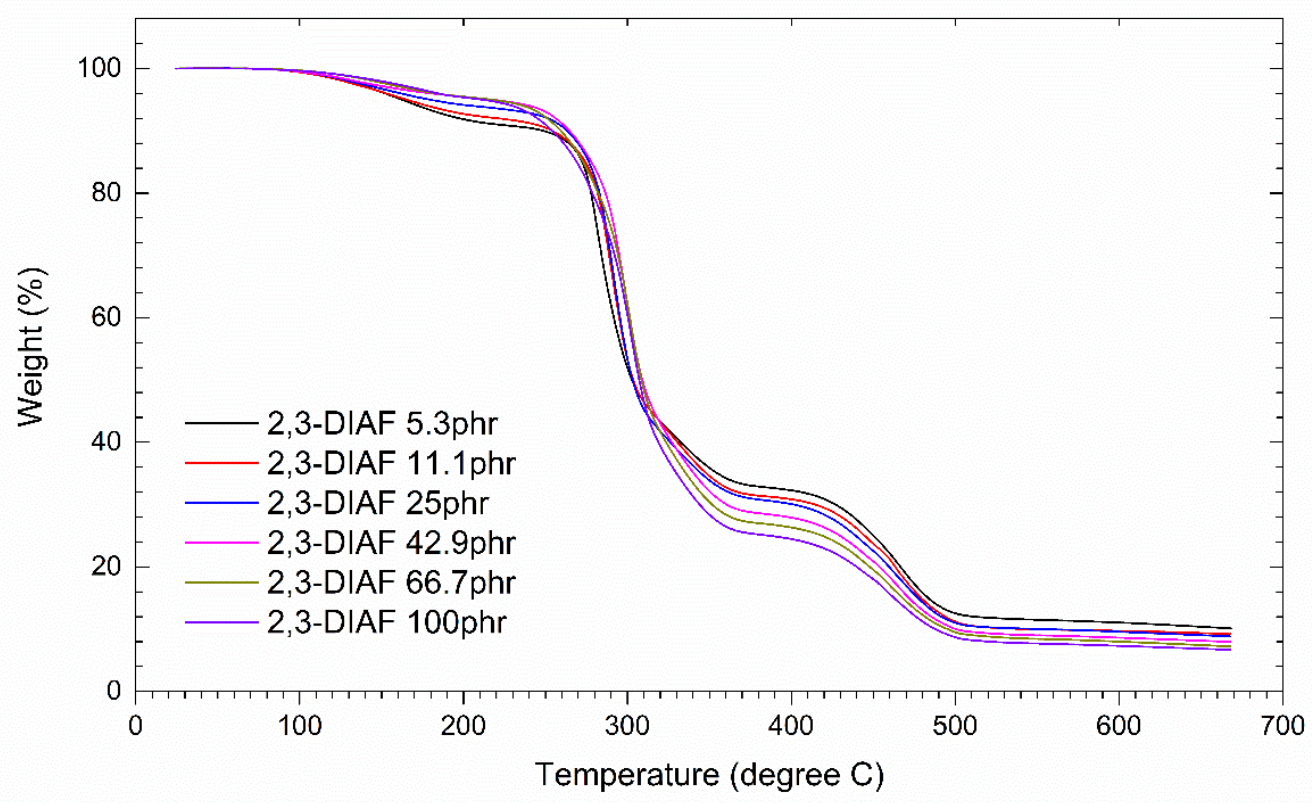

Figure S84. Thermal degradation curves of PVC-2,3-DIAF films

Table S19. Thermal degradation data of PVC-2,3-DIAF films

\begin{tabular}{lcccccc}
\hline Film & $\mathrm{T}_{5 \%}$ & $\mathrm{~T}_{10 \%}$ & $\mathrm{~T}_{25 \%}$ & $\mathrm{~T}_{50 \%}$ & $\mathrm{~T}_{75 \%}$ & \% Residue $650{ }^{\circ} \mathrm{C}$ \\
\hline 2,3-DIAF 5.3phr & 162.44 & 248.82 & 281.09 & 302.80 & 450.21 & 10.392 \\
2,3-DIAF 11.1phr & 165.41 & 254.21 & 285.74 & 303.53 & 444.80 & 9.386 \\
2,3-DIAF 25phr & 179.70 & 263.20 & 286.70 & 303.13 & 439.28 & 9.045 \\
2,3-DIAF 42.9phr & 218.01 & 264.62 & 291.54 & 309.12 & 429.69 & 8.151 \\
2,3-DIAF 66.7phr & 217.26 & 259.37 & 289.36 & 308.57 & 418.92 & 7.444 \\
2,3-DIAF 100phr & 211.74 & 254.17 & 286.31 & 307.21 & 386.85 & 6.868 \\
\hline
\end{tabular}




\section{MIGRATION RESISTANCE}

PVC-PLS 100phr films were cut into pieces, $20 \times 20 \mathrm{~mm}^{2}$. Thickness of each piece was measured individually by an electronic micrometer.

The extractability of PLS were tested, relied upon ASTM D5227-13. Square film pieces (1.0 g) were immersed in $400 \mathrm{~mL}$ of hot $n$-hexane $\left(49.5 \pm 0.5{ }^{\circ} \mathrm{C}\right.$; $95.0 \%$ purity, from Samchun, Korea) and were stirred for exact 2 hours. After extraction, films were taken out and were dried at $80 \pm$ $0.5^{\circ} \mathrm{C}$ for $2 \mathrm{~h}$. Hexane-extractable content is also expressed as percent weight loss:

$$
\text { extractability, } \%=\frac{W_{\text {initial }}-W_{\text {final }}}{W_{\text {initial }}} \times 100 \times 0.935
$$

where:

$W_{\text {initial }}, W_{\text {final }}$ are weights, before and after extraction

0.935 is correlation factor, eliminating bias between ASTM and FDA

To determine the volatility of plasticizer in the absence of a suitable heat stabilizer, ASTM D1203-10 standard (test method A) was adapted. Three or more square film pieces with relatively similar thickness, were conditioned at $23 \pm 0.5^{\circ} \mathrm{C}$, and $50 \pm 5 \% \mathrm{rh}$ for more than $20 \mathrm{~h}$, even before or after the heating process. Then in a glass beaker (d: $100 \mathrm{~mm}$ ), film pieces were sandwiched one by one between multiple layers of activated charcoal 4-14 mesh (Sigma-Aldrich, Korea). The beaker was covered in such a way that it possibly was vented. Placed in an oven, and heated at 70 $\pm 0.2^{\circ} \mathrm{C}$ for exact $24 \mathrm{~h}$. Volatile loss is expressed as percent weight loss obeying the formula:

$$
\text { volatility, } \%=\frac{W_{\text {initial, } \text { conditioned }}-W_{\text {final, conditioned }}}{W_{\text {final, }} \text { conditioned }} \times 100
$$


where:

$W_{\text {initial, } \text { conditioned }}$ is initial weight, after conditioning

$W_{\text {final, }}$ conditioned is the weight after adsorption, also after conditioning

Table S20. Table of data from extractable and volatile tests of all investigated plasticizers

\begin{tabular}{lcc|cc}
\hline & \multicolumn{2}{|c|}{ Hexane-extractable content } & \multicolumn{2}{|c}{ Volatile content } \\
\hline DOP & $\mathrm{n}=5$ & $14.14 \pm 0.92$ & $\mathrm{n}=3$ & $3.27 \pm 0.49$ \\
2,5 -DBF & $\mathrm{n}=5$ & $13.54 \pm 0.64$ & $\mathrm{n}=3$ & $12.19 \pm 0.27$ \\
2,5 -DIAF & $\mathrm{n}=3$ & $13.10 \pm 0.46$ & $\mathrm{n}=3$ & $6.89 \pm 0.40$ \\
2,3-DBF & $\mathrm{n}=5$ & $5.39 \pm 0.39$ & $\mathrm{n}=3$ & $10.05 \pm 0.83$ \\
2,3-DIAF & $\mathrm{n}=3$ & $7.89 \pm 0.65$ & $\mathrm{n}=3$ & $8.24 \pm 0.89$ \\
\hline
\end{tabular}

* where: $\quad n$ is number of test specimens

\section{REFERENCES}

1. Pavia, D. L.; Lampman, G. M.; Kriz, G. S.; Vyvyan, J. A. Introduction to Spectroscopy. 5th ed.; Cengage Learning: USA, 2015; p 239.

2. Socrates, G. Infrared and Raman: Characteristic Group Frequencies. 3rd ed.; John Wiley \& Sons: UK, 2001; p 362.

3. Larkin, P. J. Infrared and Raman Spectroscopy: Principles and Spectral Interpretation. 1st ed.; Elsevier: USA, 2011; p 786. 